medRxiv preprint doi: https://doi.org/10.1101/2021.07.05.21259649; this version posted July 6, 2021. The copyright holder for this preprint (which was not certified by peer review) is the author/funder, who has granted medRxiv a license to display the preprint in perpetuity. This article is a US Government work. It is not subject to copyright under 17 USC 105 and is also made available for use under a CCO license.

\title{
Lipid peroxidation and pathological disruption of the ApoE/Reelin-ApoER2-DAB1 axis in sporadic Alzheimer's disease
}

\author{
Christopher E. Ramsden ${ }^{1,2 \Phi}$, Gregory S. Keyes ${ }^{1}$, Elizabeth Calzada ${ }^{1}$, Mark S. Horowitz ${ }^{1}$, Jahandar \\ Jahanipour ${ }^{3}$, Fred E. Indig 4 , Daisy Zamora ${ }^{1,5}$, Andrea Sedlock ${ }^{3}$, Ruin Moaddel ${ }^{6}$, Dimitrios Kapogiannis ${ }^{7}$, \\ Dragan Maric ${ }^{3}$
}

\section{Affiliations:}

${ }^{1}$ Lipid Peroxidation Unit, Laboratory of Clinical Investigation, National Institute on Aging, NIH 251 Bayview Blvd., Baltimore, MD, 21224, USA.

${ }^{2}$ Intramural Program of the National Institute on Alcohol Abuse and Alcoholism, NIH, Bethesda, MD, 20892 , USA.

${ }^{3}$ Flow and Imaging Cytometry Core Facility, National Institute of Neurological Disorders and Stroke, NIH, Bethesda, MD, 20892, USA.

${ }^{4}$ Confocal Imaging Facility, Laboratory of Clinical Investigation, National Institute on Aging Intramural Research Program, NIH, Baltimore, MD 21224, USA.

${ }^{5}$ Department of Psychiatry, UNC School of Medicine, Chapel Hill, NC, 27599, USA

${ }^{6}$ Laboratory of Clinical Investigation, National Institute on Aging, NIH, 251 Bayview Blvd., Baltimore, MD, 21224, USA

${ }^{7}$ Human Neuroscience Unit, Laboratory of Clinical Investigation, National Institute on Aging, NIH, 251 Bayview Blvd., Baltimore, MD, 21224, USA

ф Corresponding author: Christopher Ramsden, MD, PhD., Chief, Lipid Peroxidation Unit, Laboratory of Clinical Investigation, NIA/NIH, Baltimore, MD, 21224; chris.ramsden@nih.gov

Running Title: Pathological disruption of the ApoE/Reelin-ApoE receptor-DAB1 axis in Alzheimer's disease Keywords: Lipid peroxidation; ApoE; ApoER2; Reelin; Thr19-phosphorylated PSD95, Alzheimer's disease 
medRxiv preprint doi: https://doi.org/10.1101/2021.07.05.21259649; this version posted July 6, 2021. The copyright holder for this preprint

(which was not certified by peer review) is the author/funder, who has granted medRxiv a license to display the preprint in perpetuity. This article is a US Government work. It is not subject to copyright under 17 USC 105 and is also made available for use under a CCO license.

\section{Abstract [306]}

The strong genetic link between Apolipoprotein E (ApoE) and sporadic Alzheimer's disease (AD) and the marked increase in brain lipid peroxidation observed in early AD suggest that dysfunctional lipid metabolism plays a central role in AD pathogenesis. However, specific mechanisms and targets linking ApoE and lipid peroxidation to AD are not well-defined. Here we used a combination of biochemical experiments, singlemarker immunohistochemistry (IHC), and multiplex-IHC to examine the hypothesis that synaptic ApoE receptors and their ligands ApoE and Reelin are susceptible to lipid peroxidation, and that downstream disruptions in ApoE delivery and Reelin-ApoE receptor signaling cascades contribute to the pathogenesis of sporadic AD. We found that (1) Lys and His-enriched sequences within the binding regions of ApoER2, ApoE, VLDLR and Reelin, and recombinant ApoER2, ApoE and Reelin proteins, are vulnerable to attack by aldehydic products of lipid peroxidation, generating lipid-protein adducts and acid-stable ApoE receptor-ligand complexes; (2) ApoER2, lipid peroxidation-modified ApoE, native ApoE, Reelin, and multiple downstream components of Reelin-ApoE receptor signaling cascades that govern synaptic integrity [including DAB1, Tyr232-phosphorylated DAB1, Tyr607-phosphorylated Phosphatidylinositol 3-kinase, Thr508-phosphorylated LIM kinase-1, Ser202/Thr205-phosphorylated Tau and Thr19-phosphorylated-PSD95] accumulate in the immediate vicinity of neuritic plaques and surrounding abnormal neurons, and (3) several of these ApoE/Reelin-ApoE receptor-DAB1 pathway markers positively correlate with Braak stage, A $\beta$ plaque load, and antemortem cognitive impairment. ApoE/Reelin-ApoER2-DAB1 axis pathologies were especially prominent in the dendritic compartments of the molecular layer of the dentate gyrus, cornu ammonis and subiculum, regions that receive synaptic input from the entorhinal-hippocampal projections that underlie memory formation. Taken together, these observations point toward extensive derangements in the ApoE/Reelin-ApoE receptor-DAB1 axis and provide evidence supporting a new, working hypothesis wherein lipid peroxidation-induced adduction and crosslinking of ApoE receptors and ApoE are proximate molecular events that compromise synaptic integrity and contribute to the histopathological hallmarks and cognitive deficits that characterize sporadic AD in humans. 
medRxiv preprint doi: https://doi.org/10.1101/2021.07.05.21259649; this version posted July 6, 2021. The copyright holder for this preprint

(which was not certified by peer review) is the author/funder, who has granted medRxiv a license to display the preprint in perpetuity. This article is a US Government work. It is not subject to copyright under 17 USC 105 and is also made available

\section{Introduction [740 words]} for use under a CCO license.

Late-onset, sporadic Alzheimer's disease (AD) is the most common, progressive neurodegenerative disease leading to dementia. AD is clinically characterized by prominent early impairment of memory, ${ }^{12}$ and pathologically characterized by degenerative changes and loss of neurons in Layer II of the entorhinal cortex and their (entorhinal-hippocampal or perforant pathway) projections to the molecular layer of the dentate gyrus and the hippocampus. ${ }^{3-8}$ The selective vulnerability of these neurons and their projections and synaptic connections in early AD may provide clues to the pathogenic origins of the disease. ${ }^{6}$

Inheritance of the Apolipoprotein E $\varepsilon 4(\mathrm{ApoE} 4)$ allele is the strongest genetic risk factor for sporadic AD. ${ }^{9-12}$ ApoE is the main apolipoprotein that supplies neurons with cholesterol and phospholipids, which, among other functions, are critically important for synthesis and remodeling of synaptic membranes ${ }^{13-15}$ (Fig 1). Reelin is a large, secreted glycoprotein important for brain development, but also present in the adult brain, where its downstream signaling partners [such as Disabled homolog 1 (DAB1), Phosphatidylinositol-3-kinase (PI3K), and LIM domain kinase 1 (LIMK1)] play essential roles in shaping and strengthening synapses ${ }^{16-20}$ (reviewed in ${ }^{21}$ ) (Fig 1). It is noteworthy that ApoE and Reelin are both ligands for two ApoE receptors-ApoE receptor type 2 (ApoER2) and very low-density lipoprotein receptor (VLDLR) ${ }^{22-24}$ (Fig 1) — that are localized in synapses, play essential roles in dendritic arborization and synapse formation, and regulate memory formation in rodent models. ${ }^{2125-29}$ Therefore, it is not surprising that deficits in either ApoE-mediated delivery of lipids ${ }^{13}$ 1530 or in Reelin signaling ${ }^{21}$ lead to synapse dysfunction or loss in experimental models.

A marked increase in lipid peroxidation is present even in the earliest stages of AD. ${ }^{31-38}$ However, specific mechanisms and targets linking lipid peroxidation to AD pathogenesis, in general, and mechanisms involving ApoE, in particular, are not yet well-understood. Since Lys and His residues are highly vulnerable to attack by aldehydic products of lipid peroxidation, ${ }^{39} 40$ Lys and His-enriched sequences within the ApoE, ApoER2, Reelin and VLDLR binding regions may be consequential targets for lipid peroxidation. ApoE and Reelin both use cationic double-Lys lipoprotein receptor recognition motifs to bind to overlapping or adjacent anionic sequences within the first two LDL receptor type A (LA) repeats of ApoER2 and VLDLR (Fig 1). ${ }^{41-46}$ Moreover, human, but not rodent, ApoER2 and VLDLR are unique among lipoprotein receptors in having a double-Lys motif in the transition zone between LA repeats 1 and 2 (Fig 1). ${ }^{41}$ Furthermore, this double-Lys motif in human ApoER2 and the double-Lys within the receptor binding domain of ApoE are flanked by His residues.

We hypothesized that the convergence of these four proteins and the lipid cargo of ApoE at the synaptic ApoE-ApoE receptor interface (Fig 1, shaded triangles) creates a microenvironment that is vulnerable to lipid peroxidation and that downstream disruptions in ApoE delivery and Reelin cascades contribute to AD pathogenesis. To provide biochemical proof-of-concept for this hypothesis, we first evaluated in vitro the vulnerability of Lys and His-enriched sequences within the binding domains of ApoE receptors and their ligands, and of recombinant ApoE, Reelin, and ApoER2 ectodomain proteins, to lipid aldehyde-induced 
medRxiv preprint doi: https://doi.org/10.1101/2021.07.05.21259649; this version posted July 6, 2021. The copyright holder for this preprint

(which was not certified by peer review) is the author/funder, who has granted medRxiv a license to display the preprint in perpetuity.

This article is a US Government work. It is not subject to copyright under 17 USC 105 and is also made available for use under a CCO license.

adduction and crosslinking. Next, to provide histological proof-of-concept and to explore the potential downstream consequences of putative ApoE receptor-ligand dysfunction in $\mathrm{AD}$, we developed and validated novel antibodies targeting lipid peroxidation modified-ApoE, and the aforementioned Lys and His-enriched sequences within ApoER2 and Reelin. Using conventional immunohistochemistry (IHC) and multiplex-IHC (MP-IHC) with multidomain labeling (Fig S1), we characterized immunoreactivities for these new antibodies, and a total of 52 probes targeting core components of the ApoE/Reelin-ApoE receptor-DAB1 pathways (Fig 1, a-q), classic AD pathological markers, and cytoarchitectural markers in the entorhinal cortex and hippocampal formation of rapidly-autopsied individuals without $\mathrm{AD}$ or at all stages of the pathological spectrum of $\mathrm{AD}$ progression.

We found that: (1) ApoE receptors and their ligands are indeed susceptible to lipid aldehyde-induced adduction and crosslinking; (2) lipid peroxidation-modified ApoE, native ApoE, ApoER2, Reelin, DAB1, and several core components of the Reelin signaling cascade [including Tyr607-phosphorylated PI3K (Tyr607pPI3K), Thr508-phosporylated LIMK1 (Thr508-pLIMK1) and Thr19-phosphorylated PSD95 (Thr19-pPSD95)] accumulate in the immediate vicinity of neuritic plaques and abnormal neurons; and (3) several of these markers are associated with histological progression of $\mathrm{AD}$ and the degree of antemortem cognitive impairment. Among these, Thr19-pPSD95, a marker of synaptic disassembly and long-term depression (LTD), ${ }^{47}$ exhibited striking IHC staining and particularly strong correlations with hallmark AD pathologies and cognitive impairment (Fig S2). Collective findings identify functional domains of synaptic ApoE receptors and their ligands that are vulnerable to lipid peroxidation and highlight derangements in multiple ApoE/Reelin-ApoER2-DAB1 cascade components as pathologies associated with neuritic plaques, neurofibrillary tangles, and markers of cytoskeletal destabilization and synapse disassembly in human AD. 


\section{ApoER2}

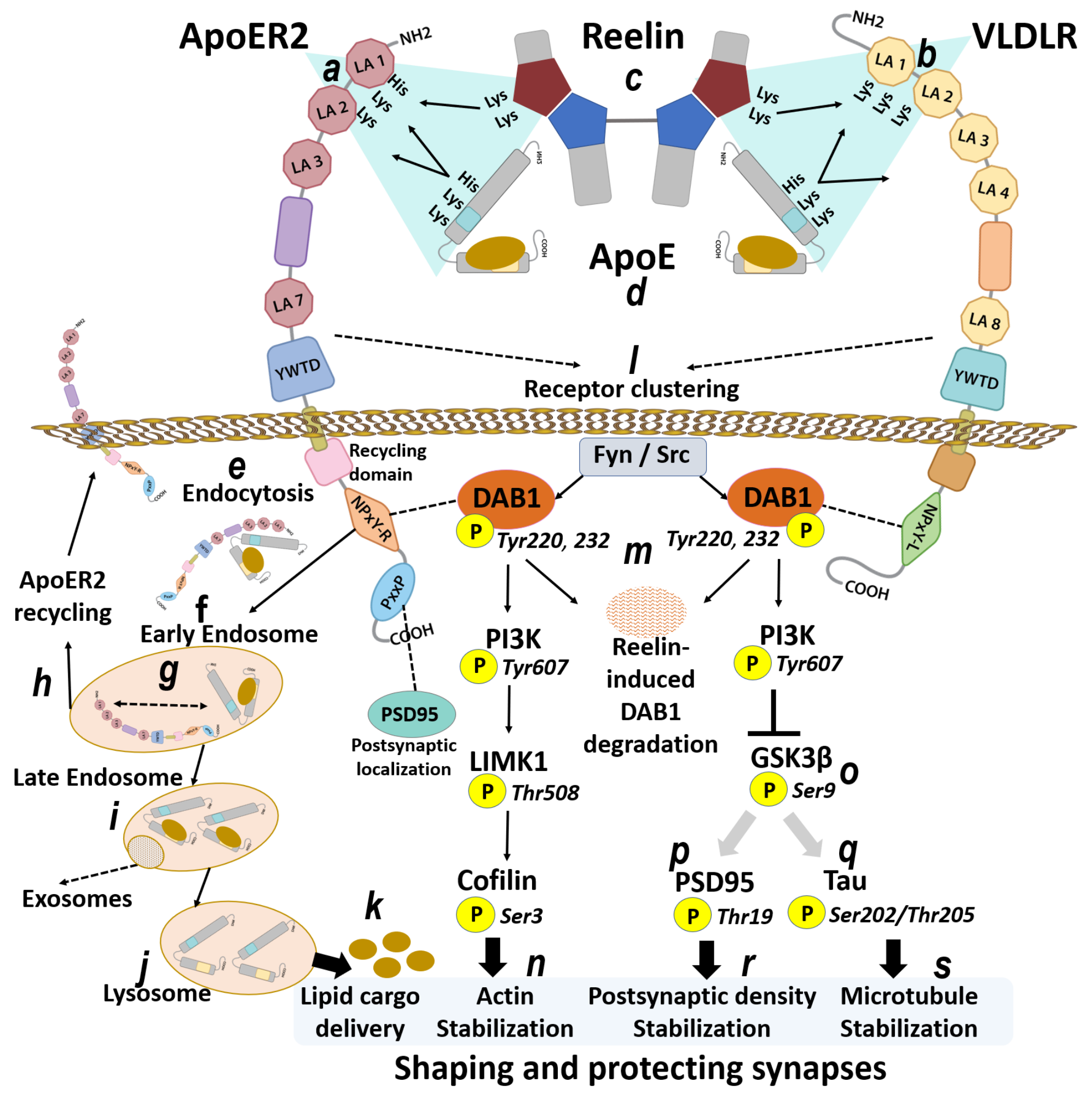


medRxiv preprint doi: https://doi.org/10.1101/2021.07.05.21259649; this version posted July 6, 2021. The copyright holder for this preprint (which was not certified by peer review) is the author/funder, who has granted medRxiv a license to display the preprint in perpetuity. This article is a US Government work. It is not subject to copyright under 17 USC 105 and is also made available

Fig 1: The ApoE/Reelin-ApoE receptor-DAB1 axis regulates synaptic integrity.

Molecular pathways central to synaptic integrity converge at the ApoE/Reelin-ApoE receptor interface (blue shading). The human versions of two synaptic ApoE receptors - ApoER2 (a) and VLDLR (b) — contain doubleLys and His residues enriched between LDL receptor repeats 1 and 2. Reelin (c) and ApoE (d) use double-Lys motifs to bind to ApoER2 and VLDLR. Upon receptor binding, ApoE particles are internalized (e) and trafficked to the early endosome (f) where acidic (pH6) conditions induce ApoE-ApoE receptor dissociation (g) followed by rapid recycling of ApoER2 to the cell surface (h). ApoE is trafficked to the late endosome (pH5.5) (i) and the lysosome $(\mathrm{pH}<5)(\mathbf{j})$, which releases lipid cargo $(\mathbf{k})$ that is required to shape synaptic membranes. Reelin binding evokes ApoE receptor clustering (l), leading to Tyr220/232 phosphorylation, activation and proteasomal degradation of DAB1 (m), and lysosomal degradation of ApoER2 (not shown). Ensuing activation of the ApoER2-PI3K-LIMK1-Cofilin arm culminates in stabilization of the actin cytoskeleton (thick arrow) (n). Activation of the VLDLR-PI3K arm of the Reelin cascade inhibits GSK3 $\beta$ by inducing its phosphorylation (thick inhibition sign) (o), which in turn inhibits Thr19-phosphorylation of PSD95 (faded arrow) (p) and Ser202/Thr205-phosphorylation of Tau (faded arrow) (q) to stabilize the postsynaptic density (thick arrow) (r) and microtubule cytoskeleton (thick arrow) (s), respectively. Efficient function of these ApoE/Reelin-ApoE receptor pathways is needed to deliver the lipid cargo and to control the signaling cascades that shape and strengthen synapses. 
medRxiv preprint doi: https://doi.org/10.1101/2021.07.05.21259649; this version posted July 6, 2021. The copyright holder for this preprint (which was not certified by peer review) is the author/funder, who has granted medRxiv a license to display the preprint in perpetuity. This article is a US Government work. It is not subject to copyright under 17 USC 105 and is also made available

\section{Results} for use under a CCO license.

\subsection{Lipid aldehyde-induced adduction and crosslinking of Lys and His-enriched sequences within ApoE} receptors and their ligands

To determine whether Lys and His-enriched sequences within the binding regions of ApoE receptors and their ligands are vulnerable to attack by aldehydic products of lipid peroxidation in vitro, sequences of four peptides (ApoER2, VLDLR, ApoE, Reelin) were incubated with malondialdehyde (MDA). ApoER2 and ApoE were additionally incubated with a mixture of aldehydes. ApoE receptors and their ligands formed a variety of aldehyde-peptide adducts (Figs 2, S3). By contrast, ApoER2 and ApoE peptide analogs lacking double-Lys motifs were resistant to adduct formation (Fig 2A, D), implying a crucial role for Lys in MDA-induced adduction. To determine the extent of reversibility of ApoER2 and ApoE peptide-aldehyde adducts, $\mathrm{pH}$ conditions of the early endosome (pH6) and lysosome $(\mathrm{pH} 4)$ were modeled. Partial, but incomplete, $\mathrm{pH}-$ dependent ( $\mathrm{pH} 4>6$ ) reversibility of adducts of ApoER2 and ApoE was observed (Fig 2B, E).

To determine if these Lys and His-enriched sequences within ApoER2 and ApoE are susceptible to lipid aldehyde-induced crosslinking, both peptides were incubated together with 4-ONE, a reactive 4-ketoaldehyde derived from peroxidation of polyunsaturated fatty acids ${ }^{48}$ and monitored with LC-MS and TOF-MS. Peaks were observed with corresponding molecular weights indicating the presence of crosslinked ApoER2-ApoE heterodimers, as well as intra-chain crosslinks within ApoE and ApoER2 peptides (Fig 2, S3). The molecular weights of each crosslinked peptide indicated addition of one 4-ONE molecule with the loss of two water molecules, suggesting the formation of pyrrole crosslinks. ${ }^{49} 50$ To determine whether His and/or Lys residues mediated the crosslinking process, the experiments were repeated using combinations of ApoER2 and ApoE analogs lacking Lys or His residues (Fig S5). We observed that both the His and Lys in ApoE, and either the His or Lys in ApoER2, were required to form these crosslinked peptides. To determine if aldehyde-peptide crosslinks can be reversed by acidic conditions modeling the lysosome, crosslinked peptides were incubated at pH4 for 24 hours. There was no detectable change in crosslinked peptides, further supporting the presence of stable pyrrole crosslinks. ${ }^{51}$ The proposed biosynthetic sequence of reactions and the chemical structures including these stable His-Lys pyrrole crosslinks are shown in Fig $\mathbf{2 G}$. 
medRxiv preprint doi: https://doi.org/10.1101/2021.07.05.21259649; this version posted July 6, 2021. The copyright holder for this preprint (which was not certified by peer review) is the author/funder, who has granted medRxiv a license to display the preprint in perpetuity. This article is a US Government work. It is not subject to copyright under 17 USC 105 and is also made available for use under a CCO license.
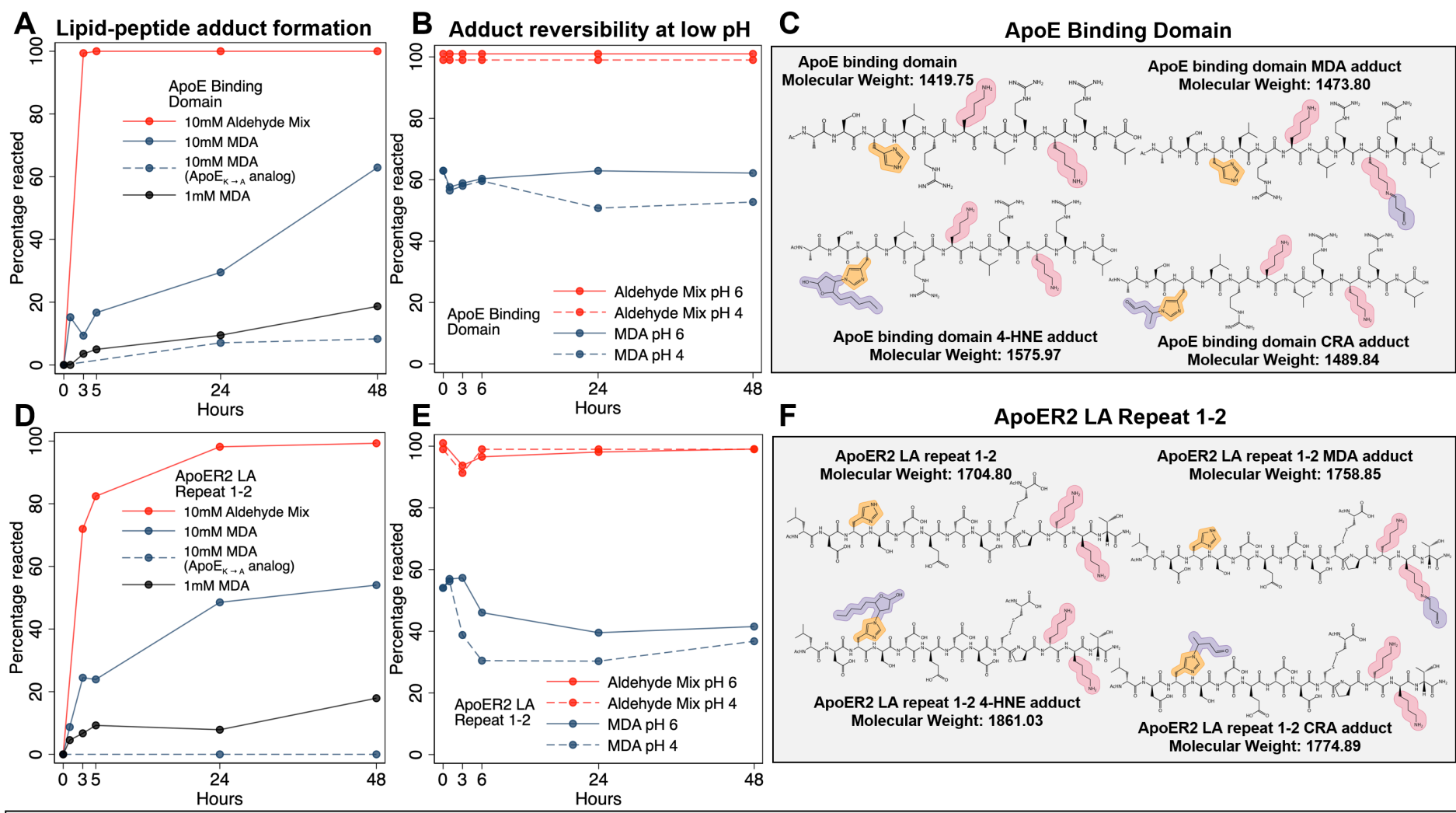

F ApoER2 LA Repeat 1-2

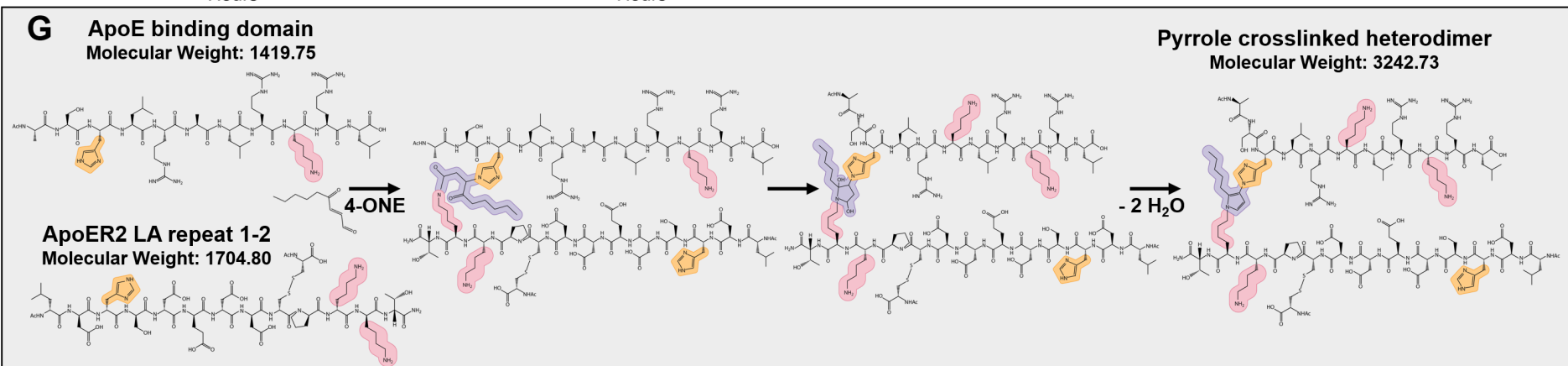

\section{H

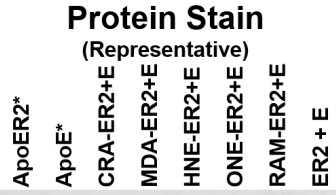

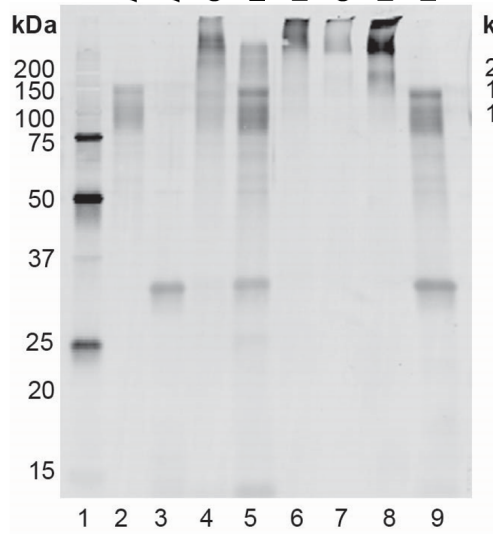

I ApoE Binding Domain

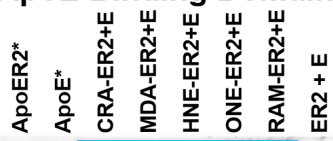

\section{J ApoER2 LA repeat 1-2}

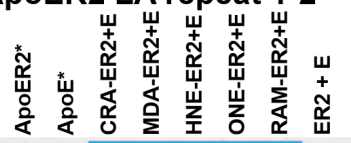

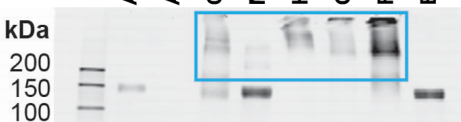

50

37

20

15

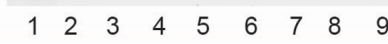

K Cu oxidized PAPC-ApoE

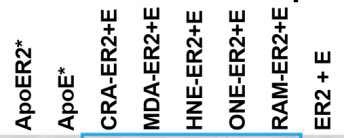

kDa
200
150
100
75
50

37

15
20

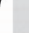

ApoER2 LA repeat 1-2 MDA adduct Molecular Weight: $\mathbf{1 7 5 8 . 8 5}$

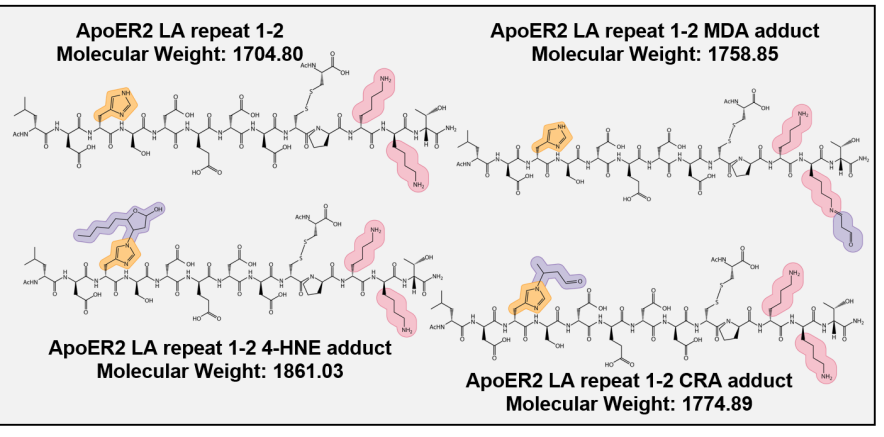
Molecular Weight: 1774.89 
medRxiv preprint doi: https://doi.org/10.1101/2021.07.05.21259649; this version posted July 6, 2021. The copyright holder for this preprint

(which was not certified by peer review) is the author/funder, who has granted medRxiv a license to display the preprint in perpetuity. This article is a US Government work. It is not subject to copyright under 17 USC 105 and is also made available for use under a CCO license.

Fig 2: Lipid aldehyde-induced adduction and crosslinking of ApoER2 and ApoE peptides and proteins detected by mass spectrometry, high molecular weight protein migration, and lipid peroxidation-specific antibodies.

Lys and His-enriched peptides within the binding regions of LA repeats 1-2 of ApoER2 (A-C) and ApoE (D-F), and their respective analogs absent the double-Lys motifs $(\mathbf{A}, \mathbf{D})$, were incubated with MDA or Reactive Aldehyde Mix (RAM) for 48 hours, followed by 48 hours of adduct reversal under conditions modeling the early endosome ( $\mathrm{pH} 6)$ or lysosome $(\mathrm{pH} 4)$. Time courses for adduct formation and $\mathrm{pH}$-dependent reversal were monitored by LC-MS. Yellow, pink and purple shading in $\mathbf{C ,} \mathbf{F}$ and $\mathbf{G}$ designate the reactive amines in His and Lys, and aldehydic adducts, respectively. Panel $\mathbf{G}$ depicts the structures and proposed sequence of molecular events mediating formation of crosslinked ApoER2-ApoE peptides. Following incubation of ApoE and ApoER2 with 4-ONE, an acid-stable crosslinked ApoER2-ApoE peptide was observed as its triple charged ion $\left(\mathrm{MW}=3242,\left[\left(\mathrm{M}+3 \mathrm{H}^{+}\right) / 3=1081\right]\right.$ and its quadruple charged ion $\left(\left[\left(\mathrm{M}+4 \mathrm{H}^{+}\right) / 4=811\right](\mathbf{F i g} \mathbf{S 5})\right.$ corresponding to a pyrrole crosslink. Parallel experiments with peptide analogs established that peptide crosslinking was dependent on His and Lys residues (Fig S4-5). Lipid aldehyde-induced crosslinking of ApoER2 plus ApoE proteins was detected by fluorescence immunoblotting against ApoE (I), ApoER2 (J), and Cu-oxidized PAPC modified-ApoE (K). 250ng of ApoER2 (lane 2) and ApoE4 (lane 3) were loaded per gel to compare with 500ng of protein from each combined lipid aldehyde reaction. Crotonaldehyde (CRA-ER2+E, Lane 4), malondialdehyde (MDA-ER2+E, Lane 5), 4-hydroxy-2-nonenal (HNE-ER2+E, lane 6), 4-oxo-2-nonenal (ONEER2+E, lane 7), Reactive Aldehyde Mixture (RAM-ER2+E, lane 8), and native ApoER2 and ApoE proteins coincubated without aldehydes (lane 8, ER2 + E) are represented in each immunoblot. $\mathbf{H}$, total protein per membrane was detected by AzureRED total protein stain. The blue box denotes higher molecular weight proteins detected. Western blots are representative of experiments performed three times. Results were also confirmed with additional ApoER2 and ApoE antibodies (Fig S6). ApoER2* indicates that the ApoER2 ectodomain protein was used; ApoE* indicates that the ApoE4 monomer was used to prevent confounding by non-aldehydic disulfide-linked dimerization. 
medRxiv preprint doi: https://doi.org/10.1101/2021.07.05.21259649; this version posted July 6, 2021. The copyright holder for this preprint

(which was not certified by peer review) is the author/funder, who has granted medRxiv a license to display the preprint in perpetuity. This article is a US Government work. It is not subject to copyright under 17 USC 105 and is also made available for use under a CCO license.

\subsection{Lipid aldehyde-induced crosslinking of ApoER2 with ApoE and Reelin proteins}

The collective observations in Section 2.1 provided proof-of-concept that double-Lys and His-enriched sequences within the binding regions of ApoE receptors and their ligands, as shown in Fig 1, are vulnerable to attack by aldehydic products of lipid peroxidation. However, these peptide sequences comprise only a fraction of all the reactive amines present in the respective full-length proteins. We next sought to determine whether the ectodomain of ApoER2, and full-length ApoE, are susceptible to lipid aldehyde-induced crosslinking and the formation of ApoER2-ApoE complexes. To model this in vitro, we incubated ApoER2 and ApoE proteins alone, together without reactive aldehydes, or together in combination with either a single lipid aldehyde or a mixture of aldehydes. In samples containing ApoER2 and ApoE alone or together without aldehydes, western blotting with two antibodies targeting ApoER2 and ApoE detected only their respective lower molecular weight bands (Fig 2H-K). By contrast, in samples containing both proteins incubated with CRA, HNE, ONE or a mixture of reactive aldehydes, higher molecular weight bands were detected by total protein stain as well as by antibodies targeting either ApoER2 or ApoE, indicating the presence of ApoER2-ApoE complexes. These findings were confirmed with two ApoER2 antibodies targeting the LA1-2 and LA3 regions, and two ApoE antibodies (Fig S6A-C, F). To model interactions between ApoER2, Reelin and reactive lipid aldehydes in vitro, we incubated the ApoER2 ectodomain and the Reelin central fragment alone, together without reactive aldehydes, or together in combination with 4-ONE. 4-ONE treatment induced formation of crosslinked ApoER2-Reelin complexes detected by total protein stain as well as by antibodies targeting ApoER2 (Fig S7).

These collective observations provide proof-of-concept that ApoER2 and its ligands ApoE and Reelin are susceptible to lipid aldehyde-induced crosslinking and formation of high molecular weight ApoER2-ligand complexes.

\subsection{Antibodies raised against lipid peroxidation-modified ApoE selectively label peroxidation-crosslinked} ApoER2-ApoE complexes and aldehyde-crosslinked ApoE multimers

Rat monoclonal antibodies were raised against lipid peroxidation-modified ApoE by incubating ApoE monomers with $\mathrm{Cu}$-oxidized 1-palmitoyl-2-arachidonoyl-sn-phosphatidylcholine (PAPC), a phospholipid that it is particularly enriched in hippocampus. ${ }^{52}$ Two clones (15E8 [PxPAPC-ApoEa], and 2B7 [PxPAPC-ApoEb]) were selected based on selective immunoreactivity for $\mathrm{Cu}$-oxidized PAPC-modified ApoE by ELISA. By western blot, both antibodies displayed immunoreactivity specific for higher molecular weight bands generated by incubation of ApoE and ApoER2 together with either 4-ONE alone, or a mixture of reactive aldehydes (Fig 2H-K, S6), or by treatment of ApoE monomers with individual reactive aldehydes (Fig S8). By contrast, neither clone detected native ApoER2, and had minimal detection of native ApoE, confirming selectivity for heteromeric ApoER2-ApoE complexes and lipid peroxidation-modified ApoE multimers. A chicken polyclonal antibody raised against ApoE monomers modified by 4-HNE (HNE-ApoE) selectively labeled high molecular weight bands consistent with aldehyde-crosslinked ApoE multimers (Fig S8F). An antibody targeting CRA- 
medRxiv preprint doi: https://doi.org/10.1101/2021.07.05.21259649; this version posted July 6, 2021. The copyright holder for this preprint

(which was not certified by peer review) is the author/funder, who has granted medRxiv a license to display the preprint in perpetuity.

This article is a US Government work. It is not subject to copyright under 17 USC 105 and is also made available for use under a CCO license.

modified ApoE (CRA-ApoE) preferentially labeled crosslinked ApoE multimers but detected ApoE monomer

(Fig S8E). Antibodies targeting ApoE modified by KOdiA or acetaldehyde detected high molecular weight bands consistent with crosslinked ApoE multimers and lower molecular weight bands for ApoE monomers (Fig S8C-D), and therefore lacked the selectivity of PxPAPC-ApoEa, PxPAPC-ApoEb and HNE-ApoE.

These observations, together with findings presented in Sections 2.1-2, provide in vitro proof-of-concept that ApoE receptors and their ligands are vulnerable to aldehyde-induced adduction and crosslinking, generating (non-disulfide linked) ApoE multimers and heteromeric ApoER2-ApoE complexes. Such adducts and complexes, if present in vivo, are predicted to disrupt ApoE internalization, ApoER2 recycling, and Reelin signaling cascades that are essential for cytoskeletal and synaptic integrity (Fig 1).

Moreover, these collective observations, in combination with results of western blot assays and RNAprotein co-detection assays (Figs S6-9) indicate that immunoprobes can be used to selectively label targets and epitopes needed to examine the putative pathological nexus (Fig 1), including PxPAPC-modified ApoE, lipid aldehyde-modified ApoE, ApoER2 LA1-2, and the Lys- and His-enriched sequence within the Reelin central fragment that mediates ApoE receptor binding.

\subsection{Accumulation of ApoE in entorhinal cortex and hippocampus is associated with neuropathological}

hallmarks and cognitive deficits in $A D$

Having demonstrated in vitro proof-of-concept for this putative pathological nexus, we sought to investigate ApoE-ApoE receptor related pathway markers in human brain specimens in anatomical regions vulnerable to AD pathogenesis. First, to examine the expression of native ApoE across the spectrum of AD progression, we used MP-IHC to immunolabel key functional domains of ApoE-including the lipoprotein receptor binding domain and lipid cargo transporting region-in entorhinal cortex and hippocampus from 24 autopsied cases of individuals who died cognitively normal, with Mild Cognitive Impairment, or AD dementia (Table 1). MP-IHC was used to label cytoarchitectural markers and classic AD pathological markers in the same sections enabling depiction of multiple hallmark AD pathologies (Fig S10). MP-IHC staining confirmed that ApoE antibodies label plaques in entorhinal cortex and adjacent to the dentate gyrus (Figs S11-12), with an antibody targeting the ApoE binding domain exhibiting a particularly strong signal within the core of neuritic plaques. Immunoreactivity for the ApoE binding domain (as percent area) in each annotated region negatively correlated with MMSE score and positively correlated with Braak stage and A $\beta$ plaque load (Table 2, Fig S2). 
medRxiv preprint doi: https://doi.org/10.1101/2021.07.05.21259649; this version posted July 6,2021 . The copyright holder for this preprint (which was not certified by peer review) is the author/funder, who has granted medRxiv a license to display the preprint in perpetuity.

This article is a US Government work. It is not subject to copyright under 17 USC 105 and is also made available for use under a CCO license.

Table 1. Clinical, neuropathological, and cognitive characteristics of age matched Alzheimer's disease, MCI, and Control specimens $(\mathbf{n}=\mathbf{2 4})$

\begin{tabular}{|c|c|c|c|}
\hline & $\begin{array}{c}\mathbf{A D} \\
(\mathrm{n}=8)\end{array}$ & $\begin{array}{l}\text { MCI } \\
(n=8)\end{array}$ & $\begin{array}{c}\text { Control } \\
(n=8)\end{array}$ \\
\hline \multicolumn{4}{|l|}{ Demographic and clinical characteristics } \\
\hline Age, $y$, mean $(S D)$ a & $82.8(5.8)$ & $83.8(4.4)$ & $81.8(6.2)$ \\
\hline Education, $\mathrm{y}$, mean (SD) & $13.8(1.8)$ & $12.2(2.3)$ & $14.1(2.8)$ \\
\hline Female, $\mathrm{n}$ & 5 & 4 & 4 \\
\hline Post mortem interval, hours, mean (SD) & $3.4(0.9)$ & $3.2(0.6)$ & $3.1(0.6)$ \\
\hline \multicolumn{4}{|l|}{ Neuropathological characteristics } \\
\hline Braak stage $(0-6)$, mean (range) & $5.5(5-6)$ & $3.8(3-4)$ & $2.1(1-3)$ \\
\hline Neurofibrillary tangle $(0-15)$, total, mean (SD) b & $13.4(1.8)$ & $7.8(1.2)$ & $3.2(2.0)$ \\
\hline Entorhinal cortex (0-3), mean (SD) & $3.0(0)$ & $3.0(0)$ & $1.8(1.0)$ \\
\hline Hippocampus (0-3), mean (SD) & $3.0(0)$ & $2.8(0.5)$ & $1.2(0.7)$ \\
\hline Temporal cortex $(0-3)$, mean (SD) & $2.9(0.4)$ & $1.4(0.7)$ & $0.2(0.5)$ \\
\hline Neuritic plaque density (0-3), mean (SD) & $3.0(0)$ & $1.4(1.5)$ & $0.6(0.7)$ \\
\hline Amyloid plaques $(0-15)$, total, mean (SD) b & $14.6(0.7)$ & $6.8(6.1)$ & $3.0(4.0)$ \\
\hline Entorhinal cortex (0-3), mean (SD) & $3.0(0)$ & $1.5(1.4)$ & $0.6(0.9)$ \\
\hline Hippocampus (0-3), mean (SD) & $2.6(0.7)$ & $0.8(0.9)$ & $0.2(0.5)$ \\
\hline Temporal cortex $(0-3)$, mean (SD) & $3.0(0)$ & $1.5(1.4)$ & $0.6(0.9)$ \\
\hline \multicolumn{4}{|l|}{ Cognitive endpoints } \\
\hline Cognitive dysfunction, y, mean (SD) & $8.5(4.1)$ & $\mathrm{n} / \mathrm{a}$ & $\mathrm{n} / \mathrm{a}$ \\
\hline MMSE $(0-30)$, mean (SD) & $13.2(7.8)$ & $26.1(2.4)$ & $28.1(0.8)$ \\
\hline Clinical Dementia Rating Sum of Boxes (0-18), mean (SD) & $11.4(5.3)$ & $1.3(1.3)$ & $0(0)$ \\
\hline Clinical Dementia Rating global score (0-3), mean (SD) & $2.1(0.9)$ & $0.4(0.2)$ & $0(0)$ \\
\hline FAST Score (1-7), mean (SD) & $5.1(0.8)$ & $2.4(1.1)$ & $1.4(0.5)$ \\
\hline Figure Recall Score (0-3), mean (SD) & $0.4(0.5)$ & $1.3(0.8)$ & $2.3(0.5)$ \\
\hline AVLT Total Learning (0-75), mean (SD) & $15.4(6.1)$ & $34.6(15.3)$ & $43.1(13.9)$ \\
\hline AVLT A6 (0-15), mean (SD) & $1.3(1.6)$ & $6.5(3.0)$ & $9.7(2.1)$ \\
\hline WMSR Digit Span Forward Score (0-12), mean (SD) & $7.5(1.3)$ & $8.4(2.0)$ & $8.4(1.8)$ \\
\hline \multicolumn{4}{|l|}{ ApoE } \\
\hline $3 / 3, \mathrm{n}$ & 5 & 3 & 5 \\
\hline $2 / 3, n$ & 1 & 5 & 1 \\
\hline $3 / 4, \mathrm{n}$ & 2 & 0 & 2 \\
\hline $2 / 2, \mathrm{n}$ & 0 & 0 & 0 \\
\hline \multicolumn{4}{|l|}{ NIA-Reagan, Likelihood of Alzheimer's disease ${ }^{c}$} \\
\hline Not Alzheimer's disease, $\mathrm{n}$ & 0 & 0 & 1 \\
\hline Low, $\mathrm{n}$ & 0 & 0 & 0 \\
\hline Intermediate, $\mathrm{n}$ & 0 & 1 & 0 \\
\hline High, $n$ & 8 & 0 & 0 \\
\hline Criteria Not Met, n & 0 & 7 & 7 \\
\hline No Dementia NOS, $\mathrm{n}$ & 8 & 8 & 8 \\
\hline No Clinpath HS, n & 8 & 8 & 8 \\
\hline No Clinpath VaD, $\mathrm{n}$ & 8 & 8 & 8 \\
\hline
\end{tabular}

${ }^{\text {a }}$ Cases that were $\geq 90$ years of age were classified by BBDP as 90 years of age or older.

b Total scores for plaques and tangles include the entorhinal, hippocampus, temporal, parietal, and frontal areas. Each was scored according to the CERAD templates ${ }^{53}$ using Campbell-Switzer silver stain, Gallyas silver stain and Thioflavin S stains.

${ }^{\mathrm{c}}$ Modified NIA-Reagan diagnosis of Alzheimer's disease based on consensus recommendations for postmortem diagnosis of Alzheimer's disease ${ }^{54}$ including neurofibrillary tangles (Braak) and neuritic plaques (CERAD). 
medRxiv preprint doi: https://doi.org/10.1101/2021.07.05.21259649; this version posted July 6, 2021. The copyright holder for this preprint (which was not certified by peer review) is the author/funder, who has granted medRxiv a license to display the preprint in perpetuity.

This article is a US Government work. It is not subject to copyright under 17 USC 105 and is also made available for use under a CCO license.

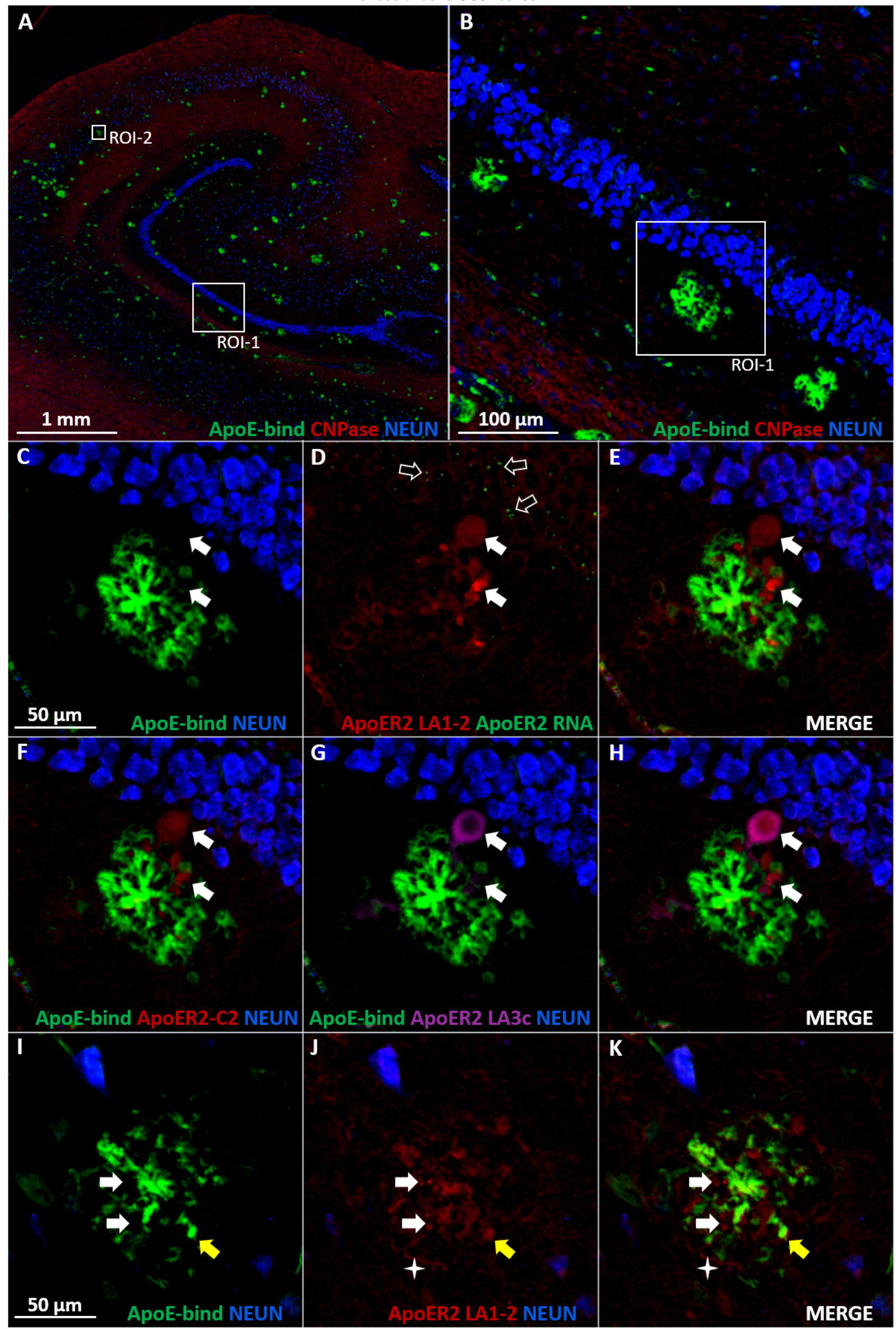


medRxiv preprint doi: https://doi.org/10.1101/2021.07.05.21259649; this version posted July 6, 2021. The copyright holder for this preprint

(which was not certified by peer review) is the author/funder, who has granted medRxiv a license to display the preprint in perpetuity. This article is a US Government work. It is not subject to copyright under 17 USC 105 and is also made available for use under a CCO license.

Fig 3. ApoER2 accumulation in proximity to ApoE-enriched plaques in the molecular layer of dentate gyrus and hippocampus. Panel $\mathbf{A}$ is a low magnification image of the hippocampus of a representative sporadic AD case (Braak Stage V, APOE3/3) that reveals extensive immunolabeling of plaques containing the receptor binding domain of ApoE (ApoE-bind, green Panels A-K). Panel B is an enlarged image containing three ApoE-enriched plaques within the molecular layer of the dentate gyrus. Panels $\mathbf{C}-\mathbf{H}$ depict a subregion containing one of these plaques (ROI-1) selected to illustrate the spatial relations between ApoER2, ApoE, and dentate granule cells, which are labeled with NEUN shown in blue. Multidomain Anti-ApoER2 immunostaining revealed enrichment of several ApoER2 domains (white arrows, Panels D-H) in proximity to ApoE-enriched plaques, with particularly strong immunolabeling of the ApoER2 LA1-2 domain (red, Panels D-E, J-K). RNA (ISH)/protein (IHC) co-detection revealed ApoER2 mRNA expression (open arrows, Panel D) within adjacent dentate granule cells indicating a likely source for ApoER2 protein labeling. In the CA2 subregion (ROI-2, Panels I-K) anti-ApoER2 LA1-2 immunolabeling demonstrated similar accumulation of ApoER2 in what appears to be dystrophic neurites (star) in the vicinity of ApoE-enriched plaques. The close proximity and colocalization of ApoER2 and ApoE immunolabeling are depicted with white arrows and yellow arrows, respectively. See Figs S13-14 for single-marker IHC images of ApoER2 LA1-2 accumulation in dentate gyrus, cornu ammonis and subiculum.

\subsection{Enrichment of ApoER2 in proximity to ApoE-enriched plaques suggests compromised ApoE receptor-} mediated internalization

Compromised ApoE binding or internalization by synaptic ApoE receptors could plausibly lead to localized, extracellular deposition of ApoE and its lipid cargo. In experimental models, the binding of ApoE to ApoER2 and VLDLR is dependent upon the cationic, double-Lys motif of ApoE and anionic regions within LA repeats 1-3 of ApoER2 and VLDLR, including the reactive amine-enriched sequences within LA repeats 1-2 of ApoER2 and VLDLR that, as shown in Figs 2 and S3-S5, are vulnerable to lipid peroxidation. To examine interactions between ApoE and ApoER2 in the plaque environment, we used multidomain MP-IHC to label the binding domain of ApoE, two sequences within the binding region of ApoER2 (ApoER2 LA1-2 and ApoER2 LA3c), and the proline-rich cytoplasmic insert (ApoER2-C2) that mediates synaptic localization and formation of ApoER2-PSD95-NMDA receptor complexes, ${ }^{23} 24265556$ in 12 cases representing the clinicopathologic spectrum of AD (Table S2). Prominent immunolabeling of ApoER2 LA1-2 was observed in close proximity to ApoE-enriched plaques in the entorhinal cortex, hippocampal formation, and dentate gyrus (Fig 3D-J). Immunoreactivities for ApoER2 LA3c and ApoER2-C2 were weaker but demonstrated substantial colocalization with ApoER2 LA1-2 (Fig 3F-H). Dendritic arbors projecting into the molecular layer of the dentate gyrus receive synaptic inputs from entorhinal-hippocampal projections that play central roles in memory. ${ }^{57-59}$ Using RNA-protein codetection, we observed prominent ApoER2 mRNA expression within dentate granule cells (Figs 3 and S9) including those adjacent to ApoE-enriched neuritic plaques, indicating a likely cellular source of plaque-associated ApoER2 protein in this region.

Using MP-IHC in these 12 cases, ApoER2 LA1-2 immunoreactivity in the hippocampus negatively correlated with MMSE score (Table 2). Using single-marker IHC, we confirmed prominent ApoER2 LA1-2 immunoreactivity in the general vicinity of plaques (Figs S13-14) and observed positive correlations between ApoER2 LA1-2 immunoreactivity and Braak stage in all three annotated regions (Table 2). 

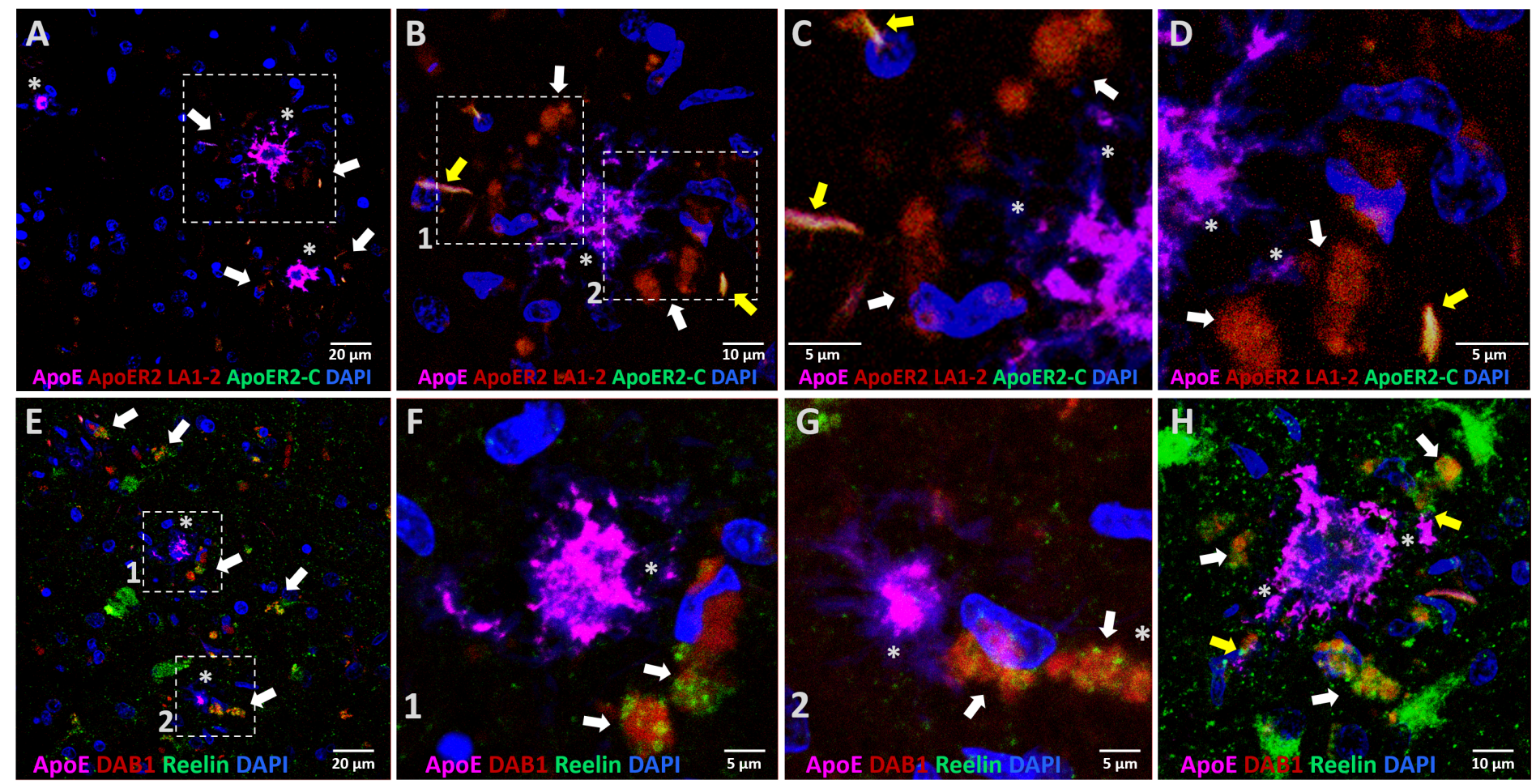

Fig 4. ApoER2, Reelin, and DAB1 enrichment in close proximity to ApoE-enriched neuritic plaques in the entorhinal cortex

Entorhinal cortex sections from a representative sporadic AD case (Braak stage V, APOE3/3) were stained with antibodies against the ApoE binding domain (magenta, A-H), the ApoER2 ligand binding region (ApoER2 LA1-2 [red, A-D]), ApoER2 cytoplasmic tail (ApoER2-C [green, A-D]), Disabled homolog 1 (DAB1 [red, E$\mathbf{H}]$ ), and Reelin (green, E-H). Plaques were visualized using intrinsic plaque autofluorescence signals (amorphous blue structures) prominent in the UV-Violet emission spectrum, whereas DAPI staining was used to visualize well-defined cell nuclei (oval or circular shaped blue objects) in the same spectrum. ApoE immunoreactive plaques $(*)$ in the entorhinal cortex are surrounded by dystrophic neurites that are strongly immunoreactive for ApoER2 LA1-2 (white arrows, A-D). A subset of ApoER2 LA1-2 immunoreactive neurites is co-labeled by the ApoER2-C antibody (yellow arrows, B-D). Accumulation of ApoER2 and DAB1 in periplaque dystrophic neurites (white arrows, B-D and E-H) despite strong extracellular Reelin immunoreactivity (yellow arrows, E-H) implies functional deficits in Reelin-ApoER2 signaling in the immediate vicinity of neuritic plaques. To facilitate three-dimensional visualization of interactions between ApoE, ApoER2, Reelin and DAB1, the confocal images shown in $(\mathbf{B}$ and $\mathbf{H})$ are provided as 3D Fiji reconstructions in Figs S15-16. 
medRxiv preprint doi: https://doi.org/10.1101/2021.07.05.21259649; this version posted July 6, 2021. The copyright holder for this preprint (which was not certified by peer review) is the author/funder, who has granted medRxiv a license to display the preprint in perpetuity. This article is a US Government work. It is not subject to copyright under 17 USC 105 and is also made available

2.6 ApoE-enriched plaques surrounded by ApoER2 and DAB1 immunoreactive dystrophic neurites in the presence of extracellular Reelin suggests compromised Reelin-ApoER2-DAB1 signaling

In experimental models, the binding of Reelin to ApoER2 induces phosphorylation of the intracellular adaptor protein DAB1, which activates a signaling cascade that stabilizes the actin cytoskeleton (reviewed in ${ }^{21}$ ) (Fig 1). ApoE and Reelin are both ligands for ApoER2, implying possible molecular interactions between ApoE and the Reelin-ApoER2-DAB1 axis. To gain insights into these interactions in neuropathological context, at higher resolution, we stained serial sections containing the entorhinal cortex of one Braak Stage V AD case with antibodies targeting the receptor binding domain of ApoE, ApoER2 LA1-2, and the ApoER2 cytoplasmic tail (ApoER2-C2) (Fig 4A-D), as well as ApoE, Reelin, and DAB1 (Fig 4E-H) and captured 40x and 100x confocal images. ApoE-enriched plaques were observed to be surrounded by dystrophic neurites that were strongly immunoreactive for the ligand binding region of ApoER2 (Fig 4A-D). Despite strong extracellular Reelin immunoreactivity (Fig 4E-H), we observed accumulation of DAB1 in peri-plaque dystrophic neurites (Fig 4EH). Since ApoER2 and DAB1 accumulate in response to impaired Reelin-ApoER2 signaling, ${ }^{23} 60$ the combination of extracellular Reelin and intraneuronal ApoER2 and DAB1 accumulation implies functional deficits in the Reelin-ApoER2-DAB1 pathway in the immediate vicinity of neuritic plaques. 3D Fiji reconstructions of these images are provided in Figs S15-16. 
medRxiv preprint doi: https://doi.org/10.1101/2021.07.05.21259649; this version posted July 6, 2021. The copyright holder for this preprint (which was not certified by peer review) is the author/funder, who has granted medRxiv a license to display the preprint in perpetuity. This article is a US Government work. It is not subject to copyright under 17 USC 105 and is also made available

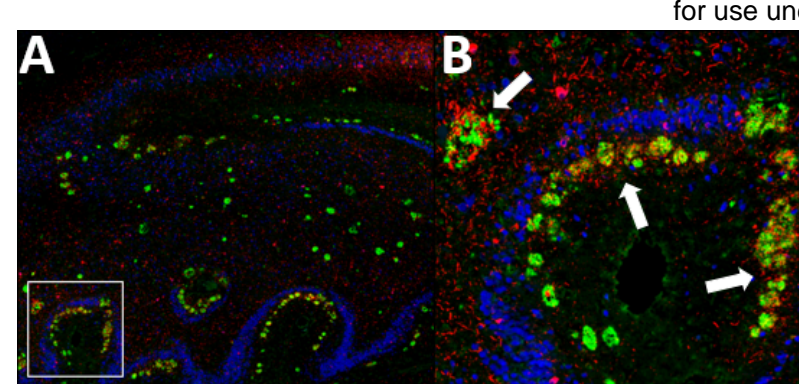

\section{ApoE píAU NEUN}

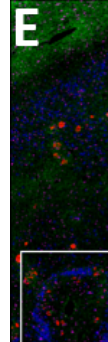

$1 \mathrm{~mm}$

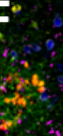
C

D
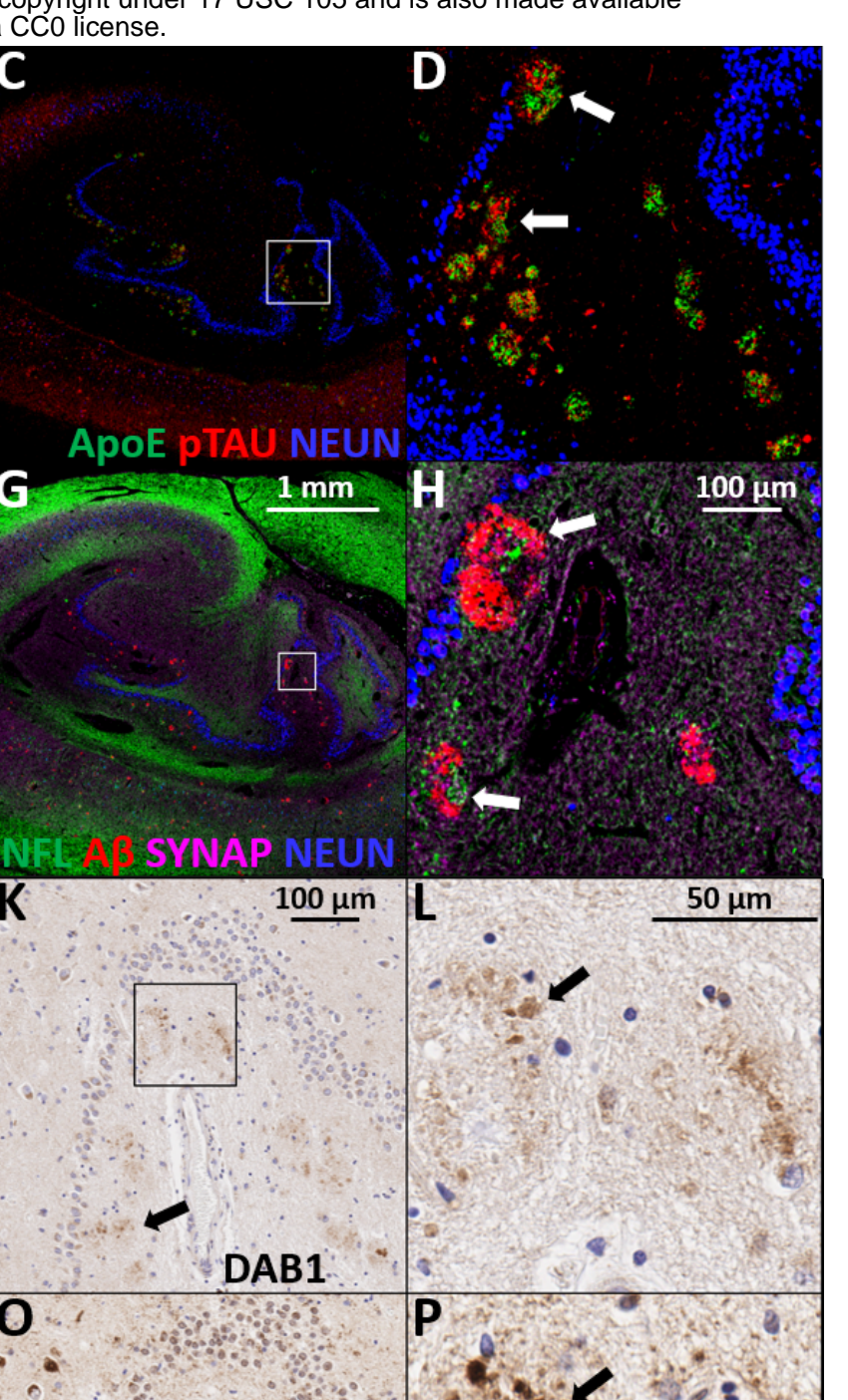

$+2$

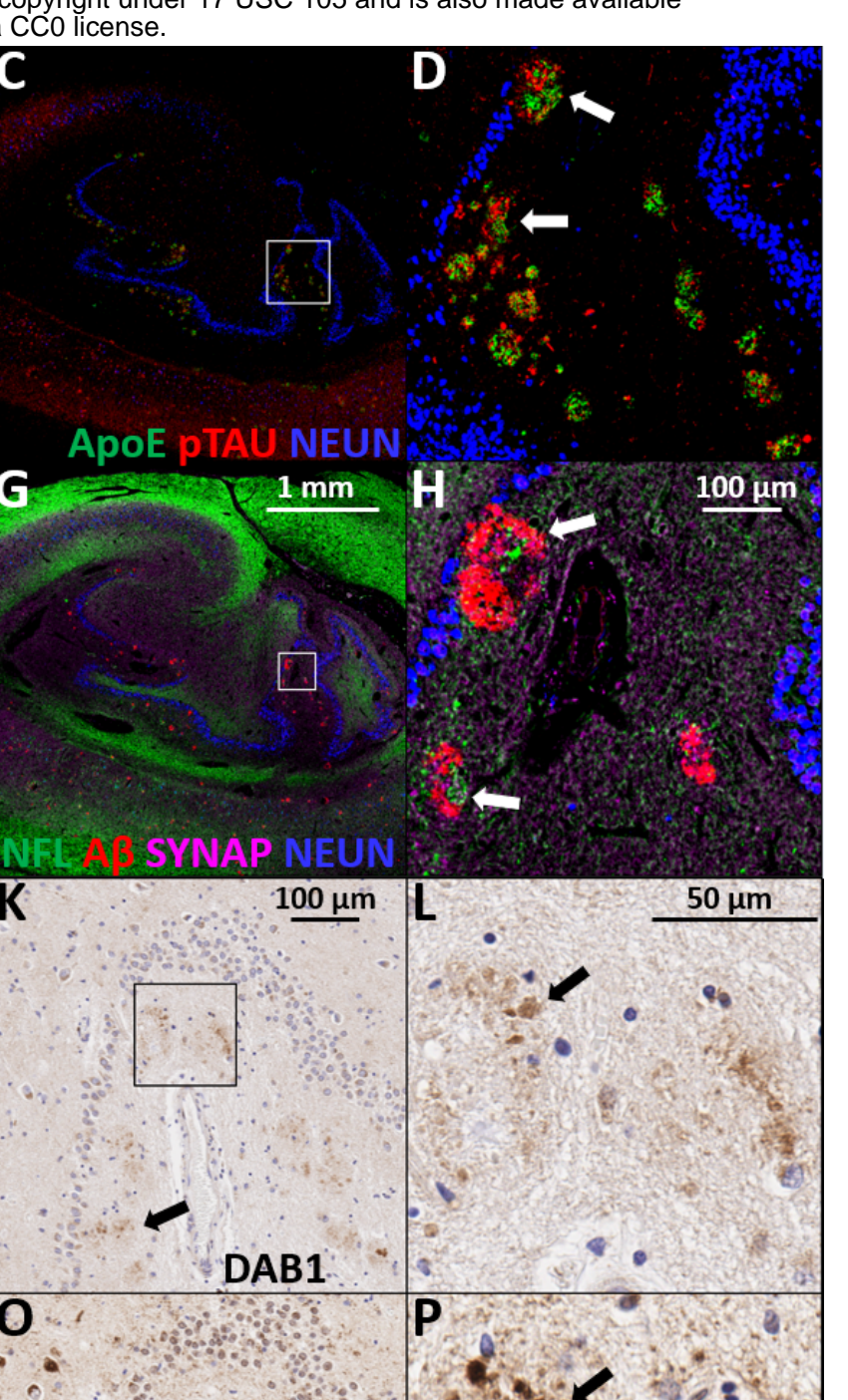

cto $85^{\circ}$ $\frac{100 \mu \mathrm{m}}{2} \mathrm{G}=\frac{1 \mathrm{~mm}}{100 \mu \mathrm{m}}$

\section{GFAP AB IBA1 NEUN}

I

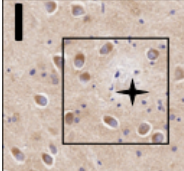

$\therefore$.

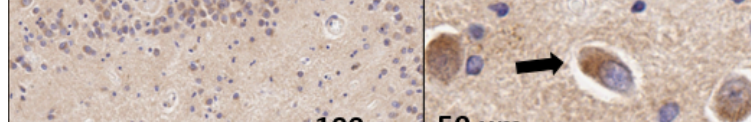

PXPAPC-ApoEa $100 \mu \mathrm{m}$ M.
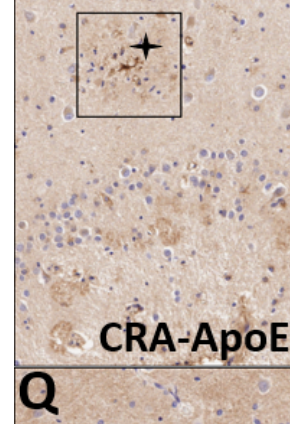

ApoER2 LA1-2

$\mathrm{U}$

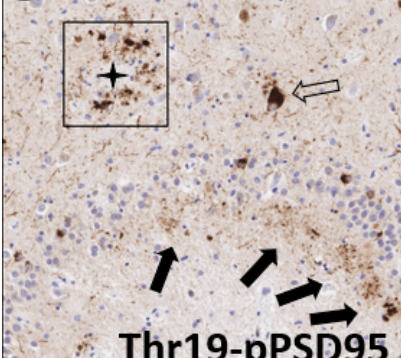

Thr19-pPSD95
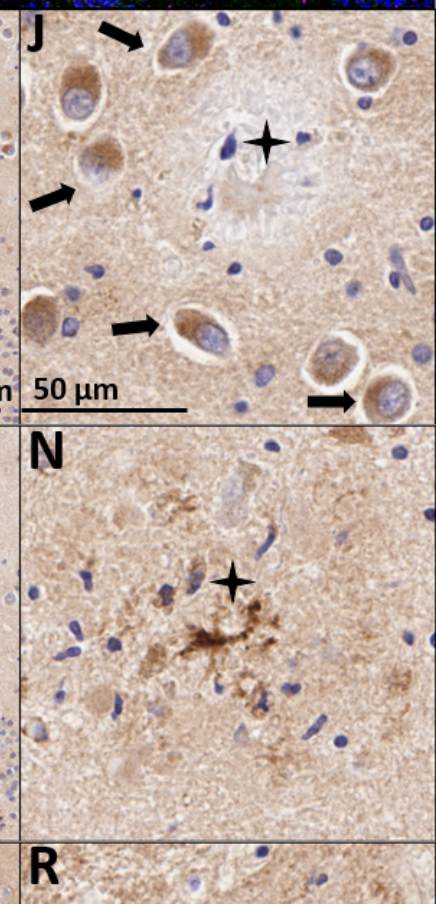
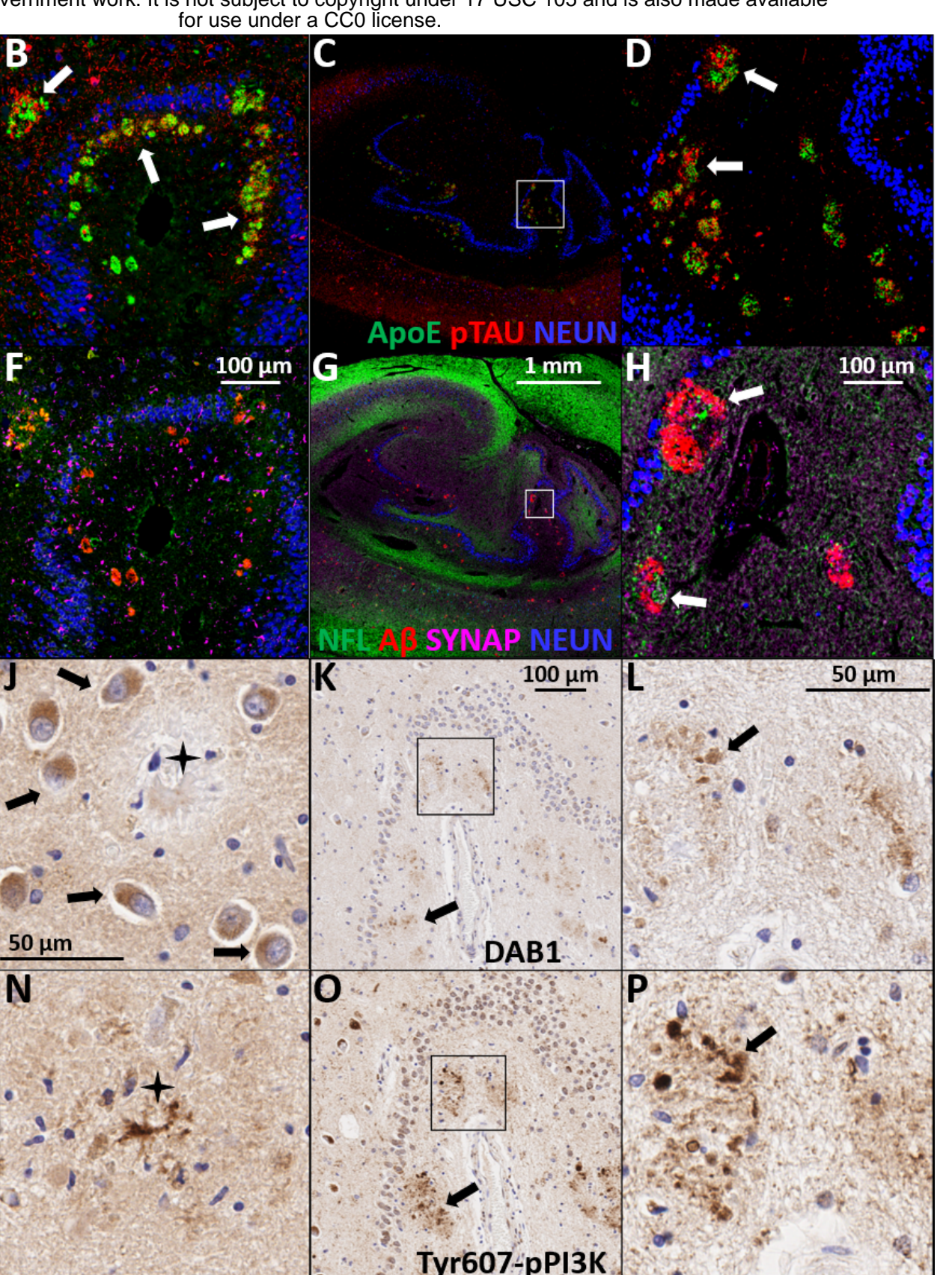

NSE
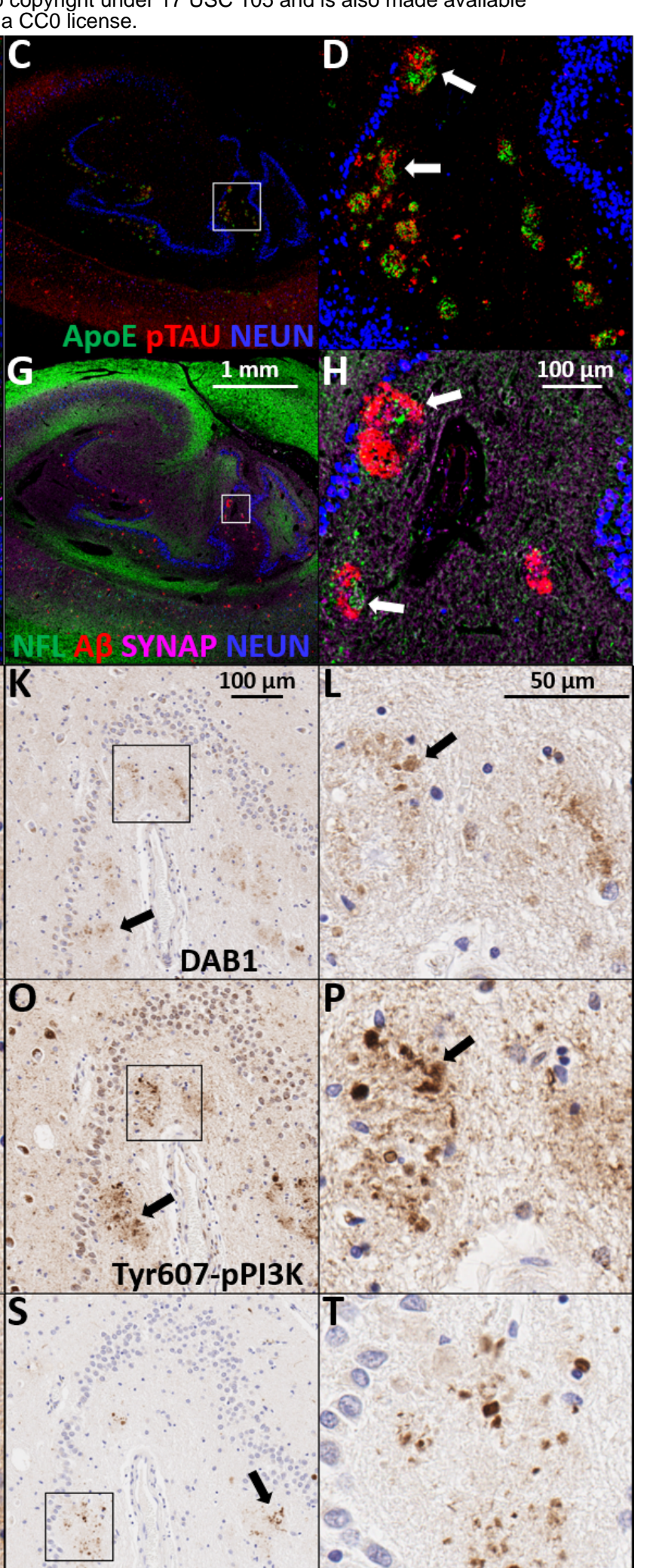

Tyr607-pPI3K

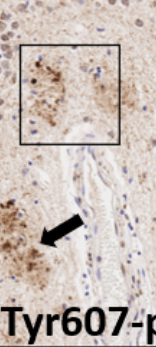


medRxiv preprint doi: https://doi.org/10.1101/2021.07.05.21259649; this version posted July 6, 2021. The copyright holder for this preprint

(which was not certified by peer review) is the author/funder, who has granted medRxiv a license to display the preprint in perpetuity.

This article is a US Government work. It is not subject to copyright under 17 USC 105 and is also made available for use under a CCO license.

Fig 5. ApoE peroxidation, Reelin-ApoER2-DAB1 cascade derangements and synaptic disassembly in sporadic AD.

Panels A-B and E-F are MP-IHC images of the hippocampus and the molecular layer of the dentate gyrus from a representative sporadic AD case (Case 1: Braak stage VI, ApoE3/4). Panels C-D and G-H are images of the hippocampus and dentate gyrus from another representative case (Case 2: Braak stage VI, ApoE3/3). Images reveal well-established $\mathrm{AD}$ pathologies including: neuritic plaques immunoreactive for $\mathrm{ApoE}, \mathrm{A} \beta$, and Ser202/Thr205-pTau, microglial plaque infiltration (IBA1, E-F), peri-plaque astrocytosis (GFAP, E-F), and accumulation of tortuous axons (NFL, G-H) and enlarged presynaptic markers (SYNP, G-H) in the vicinity of plaques. Panels, I, M, Q, U are serial sections from single-marker IHC that approximately correspond to MPIHC images in $\mathbf{B}$ and $\mathbf{F}$. J, N, R, V are further enlarged images within this region. Single-marker anti-PxPAPCApoE IHC revealed enrichment in a granular pattern, within clusters of neurons surrounding plaques (stars, I-J) that were most prominent in the hilus of the hippocampus (black arrows, I-J) but present in the dentate granule cells (open arrow, I). IHC revealed prominent immunoreactivity for CRA-ApoE within the core of plaques (stars, M-N) in the molecular layer of the dentate gyrus and the hilus of the hippocampus (N). Anti-ApoER2 LA1-2 (black arrows, Q-R) and anti-Thr19-pPSD95 (black arrows, U-V, W-X) immunolabeling revealed enrichment of both markers in the general vicinity of CRA-ApoE labeled plaques in the molecular layer of the dentate gyrus. Single-marker IHC for AD Case 2 revealed accumulations of the downstream Reelin cascade components DAB1 (K-L), Tyr607-pPI3K (O-P), Thr508-pLIMK1 (S-T), and Thr19-pPSD95 (W-X) in the vicinity of ApoE, A $\beta$, and Ser202/Thr205-pTau-positive plaques (white arrows, C-D, G-F) within the molecular layer of the dentate gyrus. 
medRxiv preprint doi: https://doi.org/10.1101/2021.07.05.21259649; this version posted July 6,2021 . The copyright holder for this preprint

(which was not certified by peer review) is the author/funder, who has granted medRxiv a license to display the preprint in perpetuity. This article is a US Government work. It is not subject to copyright under 17 USC 105 and is also made available for use under a CCO license.

2.7 Peroxidation-modified PAPC-ApoE is localized to neurons expressing markers indicative of Reelin cascade dysfunction and synapse disassembly

We hypothesized that the peroxidation of ApoE particles and ApoE receptors initiates a disease-cascade that interferes with ApoE receptor-mediated delivery of lipids and Reelin signaling in neurons (Fig 1). To examine this concept in humans, we first used single-marker IHC to immunolabel serial sections for ApoE/Reelin-ApoE receptor-DAB1 pathway markers including lipid-peroxidation modified ApoE (PxPAPCApoE and CRA-ApoE), Reelin, ApoER2 LA1-2, and multiple downstream Reelin cascade markers including DAB1, Tyr232-phosphorylated DAB1 (Tyr232-pDAB1), Tyr220-pDAB1, Tyr607-pPI3K, Thr508-pLIMK1 and Thr19-pPSD95, in 12 cases representing the clinicopathologic spectrum of AD (Table S2). To provide approximate localizations, single-marker IHC stains were completed on serial sections from cases previously labeled for cytoarchitectural and AD-related pathological markers using MP-IHC (Fig 5A-H). MP-IHC images and corresponding IHC images from two representative cases with advanced (Braak Stage VI) AD are shown in Fig 5. Anti-PxPAPC-ApoE immunolabeling revealed enrichment in a granular pattern, within clusters of neurons surrounding plaques that were most prominent in the hilus of the hippocampus (Fig 5I-J). By contrast, CRA-ApoE immunoreactivity was prominent within the core of plaques (Fig 5M-N), particularly in the molecular layer of the dentate gyrus and the hilus of the hippocampus. Anti-ApoER2 LA1-2 (Fig 5Q-R) and anti-Thr19-pPSD95 (Fig 5U-V, W-X) immunolabeling revealed enrichment of both markers in the general vicinity of CRA-ApoE-labeled plaques in the hippocampus and molecular layer of the dentate gyrus. Singlemarker IHC revealed accumulations of downstream Reelin cascade components including DAB1 (Fig 5K-L), Tyr607-pPI3K (Fig 50-P), Thr508-pLIMK1 (Fig 5S-T) and Thr19-pPSD95 (Fig 5W-X), in the general vicinity of ApoE, A $\beta$, and Ser202/Thr205-pTau-positive plaques in the molecular layer of the dentate gyrus. PxPAPC-ApoEa immunoreactivity in each annotated region negatively correlated with MMSE score (Table 2).

Next, to provide better spatial and morphological context, we used MP-IHC to label numerous ApoE/Reelin-ApoE receptor-DAB1 cascade, cytoarchitectural, and hallmark AD markers, in the same sections from 12 cases representing the clinicopathologic spectrum of AD. Images from a representative case with Braak Stage V AD are shown in Fig 6. MP-IHC revealed abundant A $\beta$-immunoreactive plaques, and prominent immunoreactivity for Thr19-pPSD95 (Fig 6A-B), implying extensive synaptic disassembly in the vicinity of neuritic plaques. As previously observed using confocal microscopy (Fig 4E-H), Reelin (C-terminal region) accumulated in the immediate vicinity of plaques (Fig 6C-F), suggesting that localized functional impairment in Reelin-ApoE receptor signaling, rather than Reelin protein depletion, accounts for the localized increases in ApoER2 and DAB1. Anti-PxPAPC-ApoE immunolabeling revealed enrichment within a subset of abnormal neurons (white arrows, Fig 6G-H, J) that were not immunoreactive for A $\beta$. Neurons that were immunoreactive for PxPAPC-ApoE showed co-labeling with markers for multiple downstream components of the Reelin kinase cascade, including Tyr232-pDAB1, Tyr220-pDAB1, Tyr607-pPI3K, Ser3-pCofilin, and Thr19-pPSD95 (white arrows, Fig 6I-L, R), as well as the dendritic marker MAP2 (Fig 6P, R). Thr19-pPSD95 immunoreactivity 
medRxiv preprint doi: https://doi.org/10.1101/2021.07.05.21259649; this version posted July 6, 2021. The copyright holder for this preprint (which was not certified by peer review) is the author/funder, who has granted medRxiv a license to display the preprint in perpetuity.

This article is a US Government work. It is not subject to copyright under 17 USC 105 and is also made available for use under a CCO license.

exhibited strong positive correlations with histological progression and antemortem cognitive impairment (Tables 2 and S3, Fig S2).

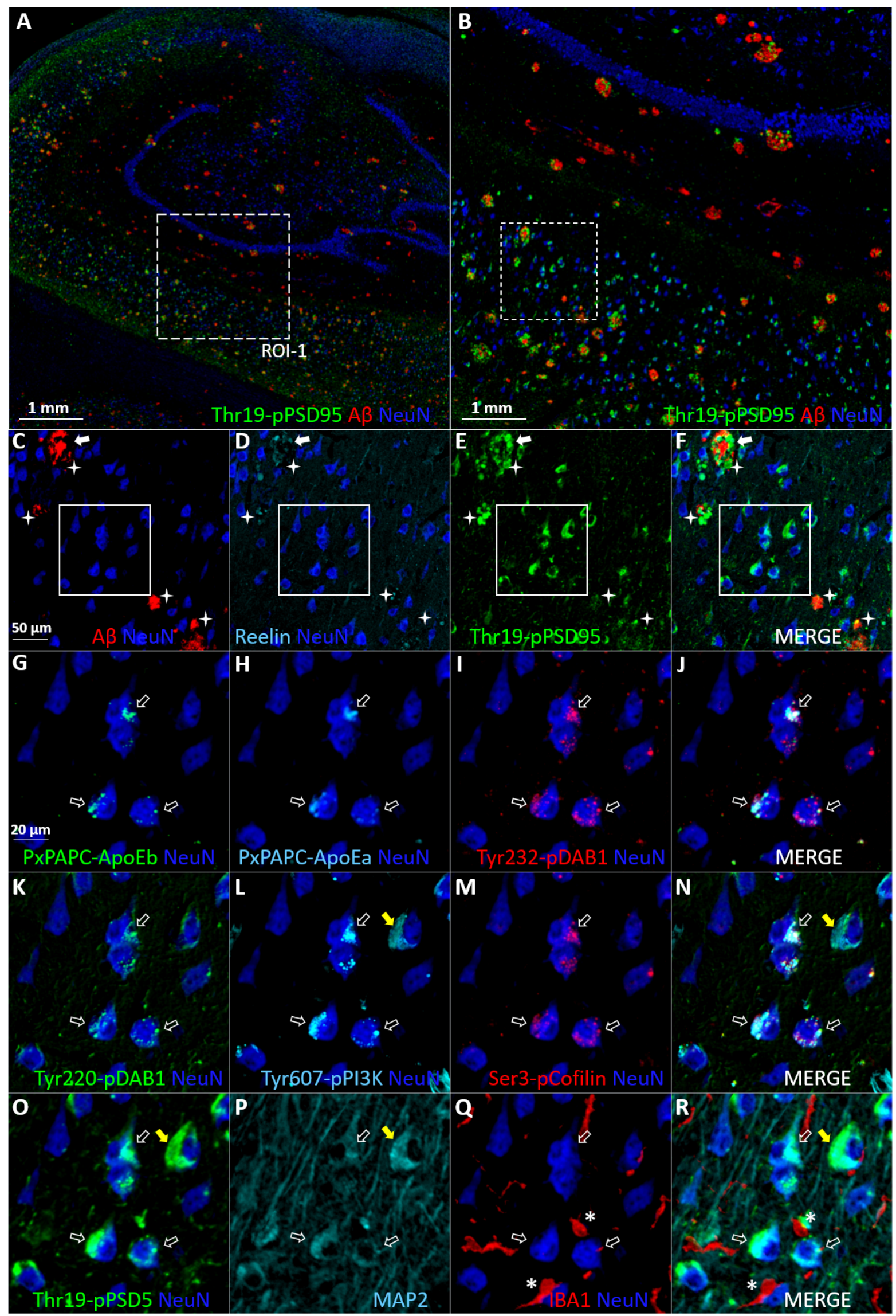


medRxiv preprint doi: https://doi.org/10.1101/2021.07.05.21259649; this version posted July 6, 2021. The copyright holder for this preprint

(which was not certified by peer review) is the author/funder, who has granted medRxiv a license to display the preprint in perpetuity. This article is a US Government work. It is not subject to copyright under 17 USC 105 and is also made available

Fig 6. Neuronal ApoE peroxidation, Reelin cascade derangements and synaptic disassembly in regions affected by sporadic AD.

Panels A and $\mathbf{B}$ are images of the hippocampus from a representative sporadic AD case (Braak stage V, ApoE3/3) which reveal abundant A $\beta$-immunoreactive plaques and prominent immunoreactivity for Thr19pPSD95, implying extensive synaptic disassembly in the vicinity of neuritic plaques. Panels $\mathbf{C}-\mathbf{F}$ are enlarged images of a representative region (ROI) within the subiculum. White boxes in $\mathbf{C}-\mathbf{F}$ demarcate a cluster of neurons surrounded by A $\beta$-immunoreactive plaques. MP-IHC revealed accumulation of extracellular Reelin (panels $\mathbf{D}, \mathbf{F}$ ) in the immediate vicinity of $A \beta$-containing plaques (depicted by stars in Panels $\mathbf{C}$-F), suggesting functional impairment in Reelin-ApoE receptor signaling rather than depletion of Reelin protein. Anti-PxPAPCApoE immunolabeling illustrated enrichment in a granular pattern, within a subset of adjacent neurons (white arrows, panels $\mathbf{G}, \mathbf{H}, \mathbf{J})$ that were not immunoreactive for $A \beta$. Neurons that were immunoreactive for PxPAPCApoE co-labeled with markers for multiple downstream components of the Reelin kinase cascade, including Tyr232-pDAB1, Tyr220-pDAB1, Tyr607-pPI3K, Ser3-pCofilin, and Thr19-pPSD95 (white arrows, panels I-L, $\mathbf{R})$, as well as the dendritic marker MAP2 (panels P, R). Thr19-pPSD95 and MAP2 were colocalized in the dendritic compartment of neurons (yellow arrows, $\mathbf{O}, \mathbf{P}, \mathbf{R}$ ). Microglia in the vicinity of affected neurons exhibited larger cell bodies with short, thick processes, morphological properties suggestive of activation (*, QR).

Jackson et al ${ }^{61}$ observed that although the vast majority of ApoE immunoreactive plaques are associated with dystrophic neurites, many A $\beta$-containing diffuse plaques lack associated ApoE. Consistent with this concept, in the vicinity of $A \beta$-containing diffuse plaques we observed minimal immunolabeling for ApoE, Tyr232-pDAB1, Tyr220-pDAB1 and Thr19-pPSD95 (Fig S17).

Collective results suggest localized interactions between neuronal ApoE peroxidation, Reelin cascade derangements, and synapse disassembly in regions affected by AD. Conventional single-marker IHC images (Figs S13-14, S18-33) reveal similar staining patterns for each ApoE/Reelin-ApoER2-DAB1 cascade marker. While these single-marker IHC images lack the cytoarchitectural and pathological context afforded by MP-IHC, they provide additional confidence in our MP-IHC observations. 
medRxiv preprint doi: https://doi.org/10.1101/2021.07.05.21259649; this version posted July 6,2021 . The copyright holder for this preprint (which was not certified by peer review) is the author/funder, who has granted medRxiv a license to display the preprint in perpetuity.

This article is a US Government work. It is not subject to copyright under 17 USC 105 and is also made available for use under a CCO license.

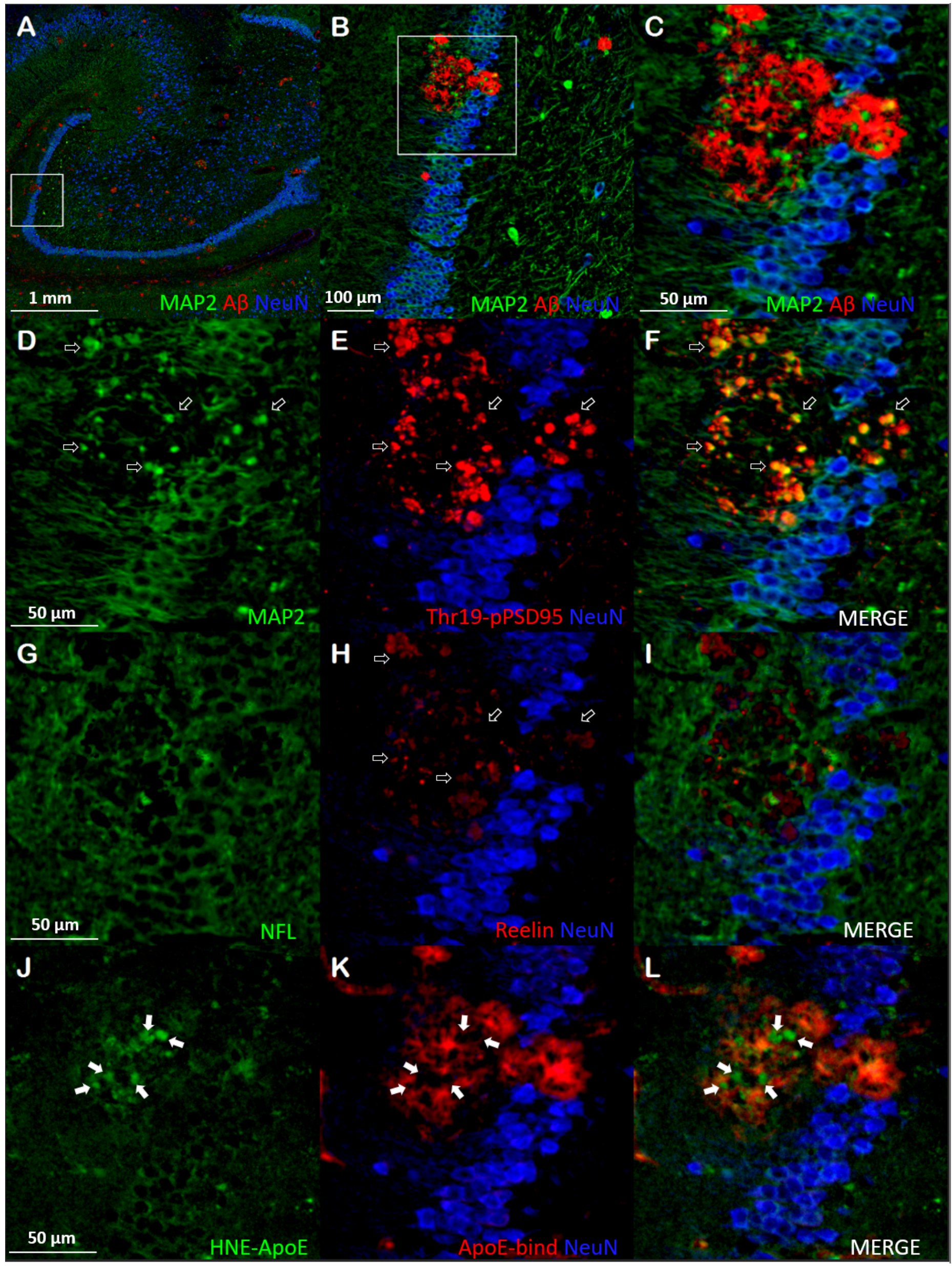


medRxiv preprint doi: https://doi.org/10.1101/2021.07.05.21259649; this version posted July 6, 2021. The copyright holder for this preprint

(which was not certified by peer review) is the author/funder, who has granted medRxiv a license to display the preprint in perpetuity. This article is a US Government work. It is not subject to copyright under 17 USC 105 and is also made available for use under a CCO license.

Fig 7. Extracellular ApoE, HNE-ApoE and Reelin converge with Thr19-pPSD95 in dendritic arbors extending into the molecular layer of the dentate gyrus

Panels $\mathbf{A}$ and $\mathbf{B}$ are images of the hippocampus from a representative sporadic AD case (Braak stage V, ApoE3/3) selected to illustrate the local environment around a representative $A \beta$ and ApoE-enriched neuritic plaque using MP-IHC. Panels C-L are enlarged images of a single representative plaque in A-B. Panels C-D illustrate prominent tortuosity of the MAP2-labeled dendritic molecular layer projections of dentate gyrus neurons, but minimal colocalization with $A \beta$ in the vicinity of neuritic plaques. Panels D-F reveal striking colocalization (open arrows) of MAP2 (green, D) with Thr19-pPSD95 (red, E) (marker of postsynaptic disassembly) within these tortuous dendritic projections. Panels G-I reveal close relations but minimal colocalization between axons (NFL [green, G, I]) and Reelin (red, H, I). Panels J-L reveal HNE-modified ApoE (HNE-ApoE [green, J, L]) immunoreactive puncta (white arrows) within the plaque core with only partial co-localization with native ApoE (red, K-L).

\subsection{ApoE/Reelin-ApoER2 axis pathologies converge in the molecular layer of the dentate gyrus}

Entorhinal-hippocampal axonal projections terminate in dendritic arbors in the molecular layer of the dentate gyrus. ${ }^{57-59}$ We previously reported enrichment of ApoER2 in close proximity to ApoE-enriched plaques

(Section 2.7, Figs 3 and 5, S13-14), suggesting convergence of ApoE/Reelin-ApoER2 axis pathologies in this region. However, it is not yet clear whether such pathologies are localized to axons or dendrites. We used MPIHC to examine spatial and morphological relationships between ApoE, HNE-ApoE, A $\beta$, Reelin, and Thr19pPSD95, and to localize each marker to plaques, MAP2-labeled dendrites, or NFL-labeled axons. An enlarged image of one representative neuritic plaque in the dentate gyrus is shown in Fig 7. As previously observed (Figs 3-6), immunolabeling showed that ApoE, Reelin, and A $\beta$ were primarily localized to extracellular plaques. Thr19-pPSD95 immunoreactivity co-localized with MAP2 in tortuous dendrites (Fig 7D-F). By contrast, NFL immunoreactivity was not colocalized with any labeled marker. Together with our previous finding of ApoER2 enrichment around ApoE-enriched plaques (Figs 3-5), these observations suggest that dendritic compartments and postsynaptic densities are a major locus for ApoE and Reelin-containing plaque-related pathologies in AD. Moreover, identification of HNE-ApoE immunoreactive puncta (white arrows in $\mathbf{J}, \mathbf{L}$ ) within the plaque core, despite minimal co-localization with native ApoE, provides further evidence that a portion of ApoE in the plaque environment is modified by lipid peroxidation. Using conventional single-marker IHC, we confirmed that CRA-ApoE and HNE-ApoE antibodies label puncta in the vicinity of the plaque core (Figs 5, S19-21), providing additional support for the MP-IHC findings. HNE-ApoE immunoreactivity positively correlated with neuritic plaque density in all three annotated regions (Table 2). 
medRxiv preprint doi: https://doi.org/10.1101/2021.07.05.21259649; this version posted July 6, 2021. The copyright holder for this preprint (which was not certified by peer review) is the author/funder, who has granted medRxiv a license to display the preprint in perpetuity. This article is a US Government work. It is not subject to copyright under 17 USC 105 and is also made available for use under a CCO license.

Table 2. Associations between immunohistochemical markers and ante-mortem cognitive performance and neuropathologic endpoints a

\begin{tabular}{|c|c|c|c|c|c|c|c|c|c|c|c|c|c|c|c|c|c|}
\hline & \multirow[b]{2}{*}{ IHC } & \multirow[b]{2}{*}{ Region } & \multicolumn{3}{|c|}{ MMSE Test Score b } & \multicolumn{3}{|c|}{ Braak Stage $\mathrm{c}$} & & Tangle & $\mathrm{tal}^{\mathrm{d}}$ & & Paque & tal ${ }^{\mathrm{e}}$ & Net & tic Plag & Density $^{f}$ \\
\hline & & & $\mathrm{n}$ & rho & p-value & $\mathbf{n}$ & rho & p-value & $\mathbf{n}$ & rho & p-value & $\mathbf{n}$ & rho & p-value & $\mathbf{n}$ & rho & p-value \\
\hline Hallmark or diagnost & AD path & gies & & & & & & & & & & & & & & & \\
\hline & & Entorhinal Cortex & 24 & -0.50 & 0.012 & 24 & 0.71 & $<0.001$ & 24 & 0.73 & $<0.001$ & 24 & 0.76 & $<0.001$ & 24 & 0.73 & $<0.001$ \\
\hline$A \beta$ & MP & Hippocampal Formation & 24 & -0.59 & 0.003 & 24 & 0.70 & $<0.001$ & 24 & 0.74 & $<0.001$ & 24 & 0.74 & $<0.001$ & 24 & 0.68 & $<0.001$ \\
\hline & & Dentate Gyrus & 24 & -0.53 & 0.008 & 24 & 0.71 & $<0.001$ & 24 & 0.70 & $<0.001$ & 24 & 0.70 & $<0.001$ & 24 & 0.64 & $<0.001$ \\
\hline & & Entorhinal Cortex & 24 & -0.50 & 0.013 & 24 & 0.66 & $<0.001$ & 24 & 0.69 & $<0.001$ & 24 & 0.68 & $<0.001$ & 24 & 0.66 & $<0.001$ \\
\hline Ser202/Thr205-pTAU & MP & Hippocampal Formation & 24 & -0.78 & $<0.001$ & 24 & 0.77 & $<0.001$ & 24 & 0.79 & $<0.001$ & 24 & 0.61 & 0.002 & 24 & 0.49 & 0.016 \\
\hline & & Dentate Gyrus & 24 & -0.71 & $<0.001$ & 24 & 0.75 & $<0.001$ & 24 & 0.77 & $<0.001$ & 24 & 0.64 & $<0.001$ & 24 & 0.53 & 0.008 \\
\hline & & Entorhinal Cortex & 9 & -0.64 & 0.06 & 12 & 0.36 & 0.25 & 12 & 0.37 & 0.23 & 12 & 0.49 & 0.11 & 12 & 0.52 & 0.08 \\
\hline Ser498-pBACE1 & MP & Hippocampal Formation & 9 & -0.81 & 0.008 & 12 & 0.58 & 0.047 & 12 & 0.61 & 0.034 & 12 & 0.70 & 0.011 & 12 & 0.60 & 0.039 \\
\hline & & Dentate Gyrus & 9 & -0.83 & 0.006 & 12 & 0.57 & 0.05 & 12 & 0.61 & 0.037 & 12 & 0.74 & 0.005 & 12 & 0.59 & 0.046 \\
\hline Ligands for ApoE rec & tors & & & & & & & & & & & & & & & & \\
\hline & & Entorhinal Cortex & 24 & -0.59 & 0.002 & 24 & 0.61 & 0.002 & 24 & 0.68 & $<0.001$ & 24 & 0.62 & 0.001 & 24 & 0.52 & 0.010 \\
\hline ApoE-bind & MP & Hippocampal Formation & 24 & -0.69 & $<0.001$ & 24 & 0.60 & 0.002 & 24 & 0.65 & $<0.001$ & 24 & 0.59 & 0.002 & 24 & 0.46 & 0.025 \\
\hline & & Dentate Gyrus & 24 & -0.73 & $<0.001$ & 24 & 0.75 & $<0.001$ & 24 & 0.76 & $<0.001$ & 24 & 0.70 & $<0.001$ & 24 & 0.55 & 0.005 \\
\hline & & Entorhinal Cortex & 9 & -0.83 & 0.006 & 12 & 0.66 & 0.020 & 12 & 0.71 & 0.010 & 12 & 0.73 & 0.007 & 12 & 0.57 & 0.05 \\
\hline ApoE-C & MP & Hippocampal Formation & 9 & -0.73 & 0.026 & 12 & 0.26 & 0.42 & 12 & 0.33 & 0.30 & 12 & 0.34 & 0.28 & 12 & 0.14 & 0.67 \\
\hline & & Dentate Gyrus & 9 & -0.81 & 0.008 & 12 & 0.31 & 0.33 & 12 & 0.35 & 0.26 & 12 & 0.33 & 0.30 & 12 & 0.11 & 0.73 \\
\hline & & Entorhinal Cortex & 9 & -0.03 & 0.95 & 12 & 0.70 & 0.011 & 12 & 0.59 & 0.044 & 12 & 0.31 & 0.32 & 12 & 0.41 & 0.18 \\
\hline Reelin-bind & Single & Hippocampal Formation & 9 & -0.07 & 0.86 & 12 & 0.63 & 0.027 & 12 & 0.57 & 0.05 & 12 & 0.36 & 0.25 & 12 & $\begin{array}{l}0.45 \\
0.35\end{array}$ & 0.26 \\
\hline & & Dentate Gyrus & 9 & 0.10 & 0.80 & 12 & 0.58 & 0.046 & 12 & 0.50 & 0.10 & 12 & 0.30 & 0.34 & 12 & 0.30 & 0.34 \\
\hline & & Entorhinal Cortex & 9 & -0.24 & 0.54 & 12 & -0.02 & 0.96 & 12 & -0.01 & 0.98 & 12 & 0.04 & 0.89 & 12 & 0.03 & 0.94 \\
\hline Reelin-C & MP & Hippocampal Formation & 9 & -0.25 & 0.51 & 12 & -0.12 & 0.70 & 12 & -0.11 & 0.74 & 12 & -0.05 & 0.89 & 12 & -0.10 & 0.76 \\
\hline & & Dentate Gyrus & 9 & -0.39 & 0.30 & 12 & -0.10 & 0.75 & 12 & -0.08 & 0.81 & 12 & 0.01 & 0.97 & 12 & -0.01 & 0.96 \\
\hline ApoE peroxidation & & & & & & & & & & & & & & & & & \\
\hline & & Entorhinal Cortex & 9 & -0.78 & 0.013 & 12 & 0.48 & 0.12 & 12 & 0.50 & 0.09 & 12 & 0.46 & 0.13 & 12 & 0.34 & 0.27 \\
\hline PxPAPC-ApoE & MP & Hippocampal Formation & 9 & -0.64 & 0.06 & 12 & 0.45 & 0.14 & 12 & 0.41 & 0.18 & 12 & 0.38 & 0.23 & 12 & 0.46 & 0.13 \\
\hline & & Dentate Gyrus & 9 & -0.71 & 0.031 & 12 & 0.32 & 0.31 & 12 & 0.28 & 0.38 & 12 & 0.33 & 0.30 & 12 & 0.36 & 0.25 \\
\hline & & Entorhinal Cortex & 8 & -0.83 & 0.011 & 10 & 0.91 & $<0.001$ & 10 & 0.91 & $<0.001$ & 10 & 0.36 & 0.30 & 10 & 0.45 & 0.20 \\
\hline PxPAPC-ApoEa & Single & Hippocampal Formation & 8 & -0.73 & 0.040 & 10 & 0.75 & 0.013 & 10 & 0.82 & 0.004 & 10 & 0.20 & 0.59 & 10 & 0.20 & 0.59 \\
\hline & & Dentate Gyrus & 8 & -0.71 & 0.05 & 10 & 0.76 & 0.011 & 10 & 0.77 & 0.010 & 10 & 0.28 & 0.43 & 10 & 0.35 & 0.33 \\
\hline & & Entorhinal Cortex & 9 & 0.03 & 0.93 & 12 & 0.44 & 0.15 & 12 & 0.42 & 0.17 & 12 & 0.53 & 0.08 & 12 & 0.62 & 0.032 \\
\hline HNE-ApoE & MP & Hippocampal Formation & 9 & -0.08 & 0.83 & 12 & 0.47 & 0.12 & 12 & 0.46 & 0.13 & 12 & 0.55 & 0.06 & 12 & 0.64 & 0.026 \\
\hline & & Dentate Gyrus & 9 & -0.08 & 0.83 & 12 & 0.47 & 0.12 & 12 & 0.46 & 0.13 & 12 & 0.55 & 0.06 & 12 & 0.64 & 0.026 \\
\hline & & Entorhinal Cortex & 9 & -0.37 & 0.32 & 12 & 0.78 & 0.003 & 12 & 0.78 & 0.003 & 12 & 0.80 & 0.002 & 12 & 0.76 & 0.004 \\
\hline ACET-ApoE & MP & Hippocampal Formation & 9 & -0.58 & 0.10 & 12 & 0.86 & $<0.001$ & 12 & 0.85 & $<0.001$ & 12 & 0.82 & 0.001 & 12 & 0.82 & 0.001 \\
\hline & & Dentate Gyrus & 9 & -0.64 & 0.06 & 12 & 0.86 & $<0.001$ & 12 & 0.84 & $<0.001$ & 12 & 0.79 & 0.002 & 12 & 0.76 & 0.004 \\
\hline ApoE receptor 2 & & & & & & & & & & & & & & & & & \\
\hline & & Entorhinal Cortex & 9 & -0.51 & 0.16 & 12 & 0.41 & 0.19 & 12 & 0.45 & 0.14 & 12 & 0.37 & 0.23 & 12 & 0.22 & 0.50 \\
\hline ApoER2 LA1-2 & MP & Hippocampal Formation & 9 & -0.73 & 0.026 & 12 & 0.43 & 0.17 & 12 & 0.45 & 0.14 & 12 & 0.58 & 0.05 & 12 & 0.42 & 0.18 \\
\hline & & Dentate Gyrus & 9 & -0.64 & 0.06 & 12 & 0.40 & 0.20 & 12 & 0.44 & 0.15 & 12 & 0.55 & 0.07 & 12 & 0.36 & 0.25 \\
\hline & & Entorhinal Cortex & 9 & 0.12 & 0.76 & 12 & 0.69 & 0.013 & 12 & 0.58 & 0.047 & 12 & 0.62 & 0.032 & 12 & 0.74 & 0.006 \\
\hline ApoER2 LA1-2 & Single & Hippocampal Formation & 9 & -0.54 & 0.14 & 12 & 0.78 & 0.003 & 12 & 0.77 & 0.003 & 12 & 0.33 & 0.29 & 12 & 0.43 & 0.17 \\
\hline & & Dentate Gyrus & 9 & -0.43 & 0.25 & 12 & 0.71 & 0.010 & 12 & 0.64 & 0.025 & 12 & 0.42 & 0.18 & 12 & 0.50 & 0.10 \\
\hline & & Entorhinal Cortex & 9 & -0.46 & 0.21 & 12 & 0.11 & 0.74 & 12 & 0.09 & 0.77 & 12 & 0.25 & 0.42 & 12 & 0.20 & 0.53 \\
\hline ApoER2 LA3 & MP & Hippocampal Formation & 9 & -0.44 & 0.24 & 12 & 0.06 & 0.85 & 12 & 0.04 & 0.91 & 12 & 0.29 & 0.36 & 12 & 0.36 & 0.25 \\
\hline & & Dentate Gyrus & 9 & -0.45 & 0.22 & 12 & -0.09 & 0.78 & 12 & -0.12 & 0.72 & 12 & 0.14 & 0.67 & 12 & 0.18 & 0.59 \\
\hline & & Entorhinal Cortex & 9 & -0.21 & 0.59 & 12 & 0.40 & 0.20 & 12 & 0.36 & 0.24 & 12 & 0.29 & 0.35 & 12 & 0.18 & 0.57 \\
\hline ApoER2 C2 & MP & Hippocampal Formation & 9 & -0.02 & 0.97 & 12 & 0.12 & 0.71 & 12 & 0.09 & 0.77 & 12 & 0.07 & 0.84 & 12 & -0.01 & 0.98 \\
\hline & & Dentate Gyrus & 9 & 0.05 & 0.90 & 12 & 0.09 & 0.79 & 12 & 0.06 & 0.85 & 12 & 0.02 & 0.96 & 12 & -0.06 & 0.85 \\
\hline Downstream signaling & ascades & & & & & & & & & & & & & & & & \\
\hline & & Entorhinal Cortex & 8 & 0.34 & 0.41 & 10 & 0.24 & 0.50 & 10 & 0.18 & 0.62 & 10 & -0.28 & 0.43 & 10 & -0.32 & 0.37 \\
\hline DAB1 & Single & Hippocampal Formation & 8 & 0.52 & 0.19 & 10 & 0.07 & 0.85 & 10 & -0.02 & 0.95 & 10 & -0.51 & 0.13 & 10 & -0.37 & 0.30 \\
\hline & & Dentate Gyrus & 8 & 0.34 & 0.41 & 10 & 0.09 & 0.81 & 10 & -0.04 & 0.92 & 10 & -0.43 & 0.22 & 10 & -0.36 & 0.31 \\
\hline & & Entorhinal Cortex & 9 & -0.25 & 0.51 & 12 & 0.43 & 0.16 & 12 & 0.45 & 0.14 & 12 & 0.11 & 0.73 & 12 & 0.38 & 0.23 \\
\hline Tyr232-pDAB1 & Single & Hippocampal Formation & 9 & -0.49 & 0.18 & 12 & 0.49 & 0.10 & 12 & 0.45 & 0.14 & 12 & 0.26 & 0.42 & 12 & 0.46 & 0.14 \\
\hline & & Dentate Gyrus & 9 & -0.07 & 0.86 & 12 & 0.41 & 0.18 & 12 & 0.34 & 0.28 & 12 & -0.14 & 0.66 & 12 & 0.13 & 0.70 \\
\hline & & Entorhinal Cortex & 9 & -0.41 & 0.27 & 12 & 0.59 & 0.045 & 12 & 0.63 & 0.028 & 12 & 0.33 & 0.30 & 12 & 0.42 & 0.17 \\
\hline Tyr607-pPI3K & Single & Hippocampal Formation & 9 & -0.23 & 0.56 & 12 & 0.58 & 0.046 & 12 & 0.60 & 0.041 & 12 & 0.13 & 0.68 & 12 & 0.29 & 0.36 \\
\hline & & Dentate Gyrus & 9 & 0.38 & 0.32 & 12 & 0.26 & 0.42 & 12 & 0.21 & 0.50 & 12 & -0.25 & 0.43 & 12 & -0.03 & 0.93 \\
\hline & & Entorhinal Cortex & 9 & -0.41 & 0.28 & 12 & 0.61 & 0.034 & 12 & 0.66 & 0.020 & 12 & $\frac{-0.25}{0.71}$ & 0.010 & 12 & $\begin{array}{l}-0.05 \\
0.44\end{array}$ & 0.15 \\
\hline Thr508-pLIMK1 & MP & Hippocampal Formation & 9 & -0.31 & 0.42 & 12 & 0.65 & 0.022 & 12 & 0.68 & 0.014 & 12 & 0.66 & 0.019 & 12 & 0.41 & 0.18 \\
\hline & & Dentate Gyrus & 9 & 0.29 & 0.45 & 12 & 0.36 & 0.24 & 12 & 0.40 & 0.20 & 12 & 0.36 & 0.25 & 12 & 0.11 & 0.73 \\
\hline & & Entorhinal Cortex & 9 & -0.73 & 0.026 & 12 & 0.65 & 0.023 & 12 & 0.68 & 0.016 & 12 & 0.47 & 0.12 & 12 & 0.58 & 0.047 \\
\hline Thr508-pLIMK1 & Single & Hippocampal Formation & 9 & -0.45 & 0.22 & 12 & 0.65 & 0.021 & 12 & 0.59 & 0.044 & 12 & 0.44 & 0.15 & 12 & 0.34 & 0.27 \\
\hline & & Dentate Gyrus & 9 & -0.23 & 0.54 & 12 & 0.54 & 0.07 & 12 & 0.48 & 0.12 & 12 & 0.23 & 0.46 & 12 & 0.21 & 0.51 \\
\hline & & Entorhinal Cortex & 9 & 0.07 & 0.86 & 12 & 0.41 & 0.19 & 12 & 0.48 & 0.12 & 12 & 0.43 & 0.16 & 12 & 0.26 & 0.41 \\
\hline Ser3-pCofilin & MP & Hippocampal Formation & 9 & 0.42 & 0.26 & 12 & 0.28 & 0.38 & 12 & 0.36 & 0.25 & 12 & 0.07 & 0.84 & 12 & 0.31 & 0.33 \\
\hline & & Dentate Gyrus & 9 & 0.59 & 0.09 & 12 & 0.26 & 0.42 & 12 & 0.34 & 0.28 & 12 & 0.11 & 0.73 & 12 & 0.14 & 0.67 \\
\hline & & Entorhinal Cortex & 9 & -0.85 & 0.004 & 12 & 0.86 & $<0.001$ & 12 & 0.88 & $<0.001$ & 12 & 0.88 & $<0.001$ & 12 & 0.79 & 0.002 \\
\hline Thr19-pPSD95 & MP & Hippocampal Formation & 9 & -0.97 & $<0.001$ & 12 & 0.92 & $<0.001$ & 12 & 0.92 & $<0.001$ & 12 & 0.89 & $<0.001$ & 12 & 0.80 & 0.002 \\
\hline & & Dentate Gyrus & 9 & -0.85 & 0.004 & 12 & 0.83 & $<0.001$ & 12 & 0.80 & 0.002 & 12 & 0.67 & 0.018 & 12 & 0.52 & 0.08 \\
\hline
\end{tabular}

a Spearman correlations have three levels of shading based on $\mathrm{p}$-values: light, $0.05 \geq \mathrm{p}>0.01$; moderate, $0.01 \geq \mathrm{p} \geq 0.001$; dark, $\mathrm{p}<0.001$.

${ }^{b}$ Folstein Mini Mental State Examination score (0-30) obtained most proximal to death; includes MMSE scores obtained through BBDP research clinical visits and by review of private medical records. ${ }^{62}$

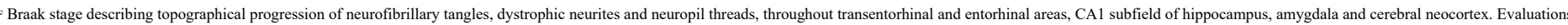

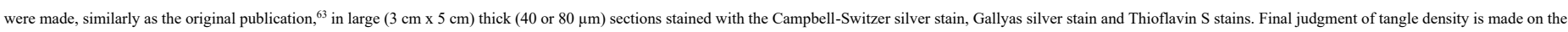

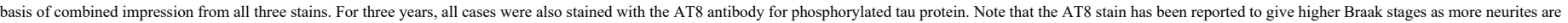
apparent. ${ }^{64}$

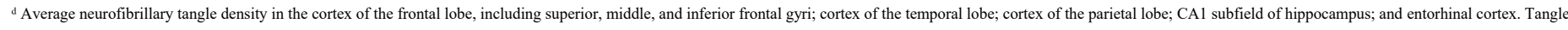
density scored according to the CERAD templates, ${ }^{53}$ as described for the Braak stage above.

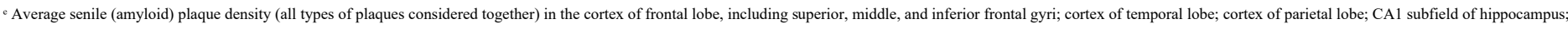

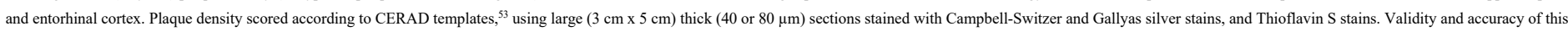

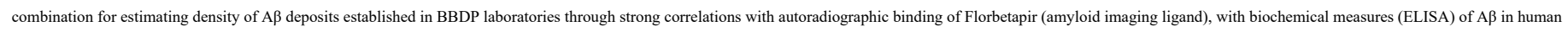
cerebral cortex extracts and with quantitative measures (percentage of section area occupied) of an immunohistochemical stain for $\mathrm{A} \beta .^{65}$

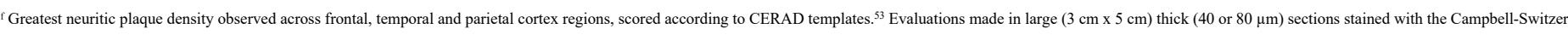
silver stain, Gallyas silver stain and Thioflavin S stains. Final judgment of plaque density made on the basis of the combined impression from all three stains. ${ }^{65}$ 
medRxiv preprint doi: https://doi.org/10.1101/2021.07.05.21259649; this version posted July 6, 2021. The copyright holder for this preprint

(which was not certified by peer review) is the author/funder, who has granted medRxiv a license to display the preprint in perpetuity.

This article is a US Government work. It is not subject to copyright under 17 USC 105 and is also made available for use under a CCO license.

2.9 ApoE/Reelin cascade derangements intersect with Tau phosphorylation in the immediate vicinity of neuritic plaques and adjacent neurons

In rodent and in vitro models, ApoE/Reelin-ApoE receptor-DAB1 signaling decreases Tau phosphorylation and generation of $A \beta,{ }^{66-72}$ suggesting that impaired Reelin signaling may contribute to hallmark AD pathologies. However, relationships between core components of the Reelin-ApoE receptor-DAB1 axis and Tau phosphorylation are largely unexplored in humans. To address this gap, we used MP-IHC to immunolabel Ser202/Thr205-pTau alongside Thr508-pLIMK1, a core component of the Reelin-ApoER2-DAB1-actin cytoskeleton pathway. 
medRxiv preprint doi: https://doi.org/10.1101/2021.07.05.21259649; this version posted July 6, 2021. The copyright holder for this preprint (which was not certified by peer review) is the author/funder, who has granted medRxiv a license to display the preprint in perpetuity.

This article is a US Government work. It is not subject to copyright under 17 USC 105 and is also made available for use under a CCO license.

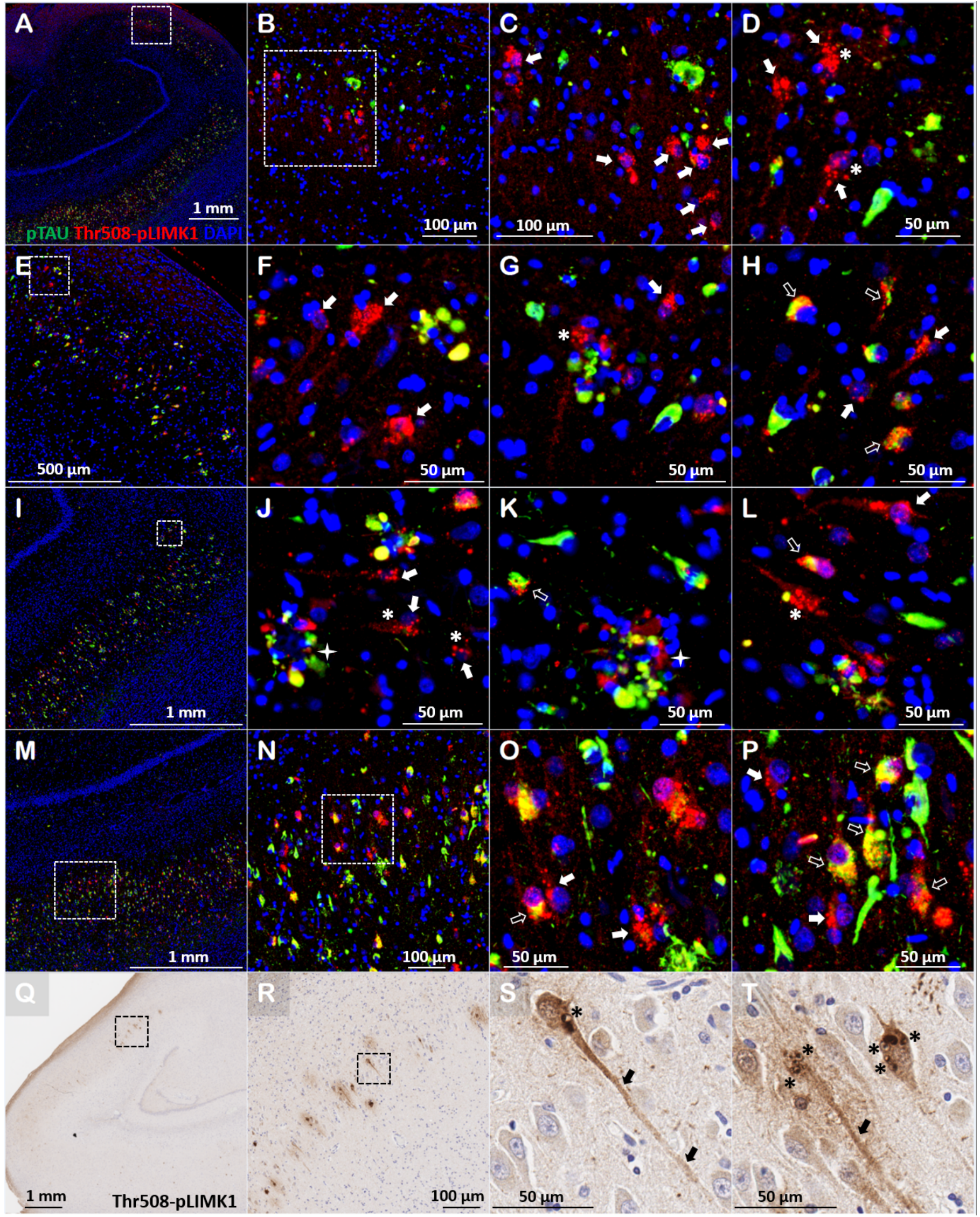


medRxiv preprint doi: https://doi.org/10.1101/2021.07.05.21259649; this version posted July 6, 2021. The copyright holder for this preprint

(which was not certified by peer review) is the author/funder, who has granted medRxiv a license to display the preprint in perpetuity. This article is a US Government work. It is not subject to copyright under 17 USC 105 and is also made available for use under a CCO license.

Fig 8. Thr508-pLIMK1 and Ser202/Thr205-pTau accumulate within neuronal subpopulations in cornu ammonis and subiculum in sporadic AD.

MP-IHC and single marker IHC were used to stain medial temporal lobe sections from a representative case with advanced AD (Braak Stage V, ApoE3/3, A-P) and an age-matched non-AD control (Braak Stage I, ApoE3/3, Q-T), respectively. Panels A, E, I, M are global MP-IHC images of the hippocampus and subiculum which reveal extensive Thr508-pLIMK1 and Ser202/Thr205-pTau (pTAU) immunolabeling of neurons and neuritic plaques. Thr508-pLIMK1 and Ser202/Thr205-pTau were observed to accumulate in the same affected regions with only partial cellular overlap (panels C-P). Neuronal subpopulations with prominent Thr508pLIMK1 labeling but little or no Ser202/Thr205-pTau, and neurons with both markers, are depicted with white arrows and open arrows, respectively. Thr508-pLIMK1 immunolabeling using MP-IHC revealed circular, granular intraneuronal inclusions predominantly localized to soma and proximal neurites (depicted by * in panels D, G, J, L). Anti-Thr508-pLIMK1 single-marker IHC revealed strong labeling of dense core granules within cytoplasmic vacuolar structures in hippocampal neurons (depicted by * in panels $\mathbf{S}$ and $\mathbf{T}$ ).

Representative MP-IHC images from a Braak Stage V AD case reveal strong regional co-expression of Thr508-pLIMK1 and Ser202/Thr205-pTau with both markers localized to neuritic plaques and subsets of surrounding neurons (Figs 8 and S30); however only partial cellular co-localization was observed. Since LIMK1 and TAU are downstream components of the Reelin-ApoER2-DAB1 and Reelin-VLDLR-DAB1 signaling cascades (Fig 1) that stabilize actin ${ }^{73} 74$ and microtubules, ${ }^{75}$ respectively, our findings imply parallel deficits in both cytoskeletons that can potentially be linked back to the disruption of Reelin signaling. This interpretation is consistent with our observation that extracellular Reelin accumulates in close proximity to a subset of ApoE-enriched neuritic plaques (Figs 3-6). Thr508-pLIMK1 immunoreactivity in the entorhinal cortex and hippocampus positively correlated with the Braak stage and A $\beta$ plaque load (Table 2).

\subsection{ApoE and Reelin containing plaques intersect with classic A $\beta$-related pathologies}

Core components of the Reelin signaling cascade regulate synthesis and deposition of $A \beta .{ }^{69}$ Moreover, Reelin has been observed to counter neurotoxic effects of $A \beta^{69} 7172$. Enlarged endo-lysosomal structures within dystrophic neurites — including late endosomes and lysosomes — are recognized as a major site of Beta-site A $\beta$ PP cleaving enzyme 1 (BACE1)-mediated conversion of A $\beta P P$ to $A \beta .{ }^{76-80}$ Despite these suggestive observations, relationships between Reelin, BACE1 and $\mathrm{A} \beta$ are not well understood in humans.

We used MP-IHC to examine relationships between A $\beta$ pathway markers (A $\beta$, Ser498-pBACE1), CD63 (marker of late endosomes), ApoE, and Reelin in AD. Representative images from a Braak Stage V AD case are shown in Fig 9. Enlarged images within the CA3 region $(\mathbf{C}-\mathbf{N})$ show that A $\beta$, ApoE, Reelin, and Thr19pPSD95positive neuritic plaques are surrounded by neurons and dystrophic neurites that co-label for Ser498-pBACE1, CD63 and phospholipid peroxidation-modified ApoE (PxPAPC-ApoEb). The tortuosity observed for MAP2 and NFL immunolabeling is consistent with well-established altered neuritic morphology that has been observed in and around plaques ${ }^{81} 82$ and is a proposed site of A $\beta$ synthesis. ${ }^{7677}$ Moreover, Ser498-pBACE1 immunolabeling in hippocampus and dentate gyrus positively correlated with $\mathrm{A} \beta$ plaque load and negatively correlated with antemortem MMSE. Single-marker IHC provides further evidence of enrichment of CD63, 
medRxiv preprint doi: https://doi.org/10.1101/2021.07.05.21259649; this version posted July 6, 2021. The copyright holder for this preprint

(which was not certified by peer review) is the author/funder, who has granted medRxiv a license to display the preprint in perpetuity.

$$
\begin{array}{r}
\text { This article is a US Government work. It is not subject to copyright under } \\
\text { for use under a CCO license. }
\end{array}
$$

LAMP1 (lysosomal marker), lipid-peroxidation-modified ApoE, and Ser498-pBACE1 in regions enriched with neuritic plaques (Figs S18-21 and S33-35).

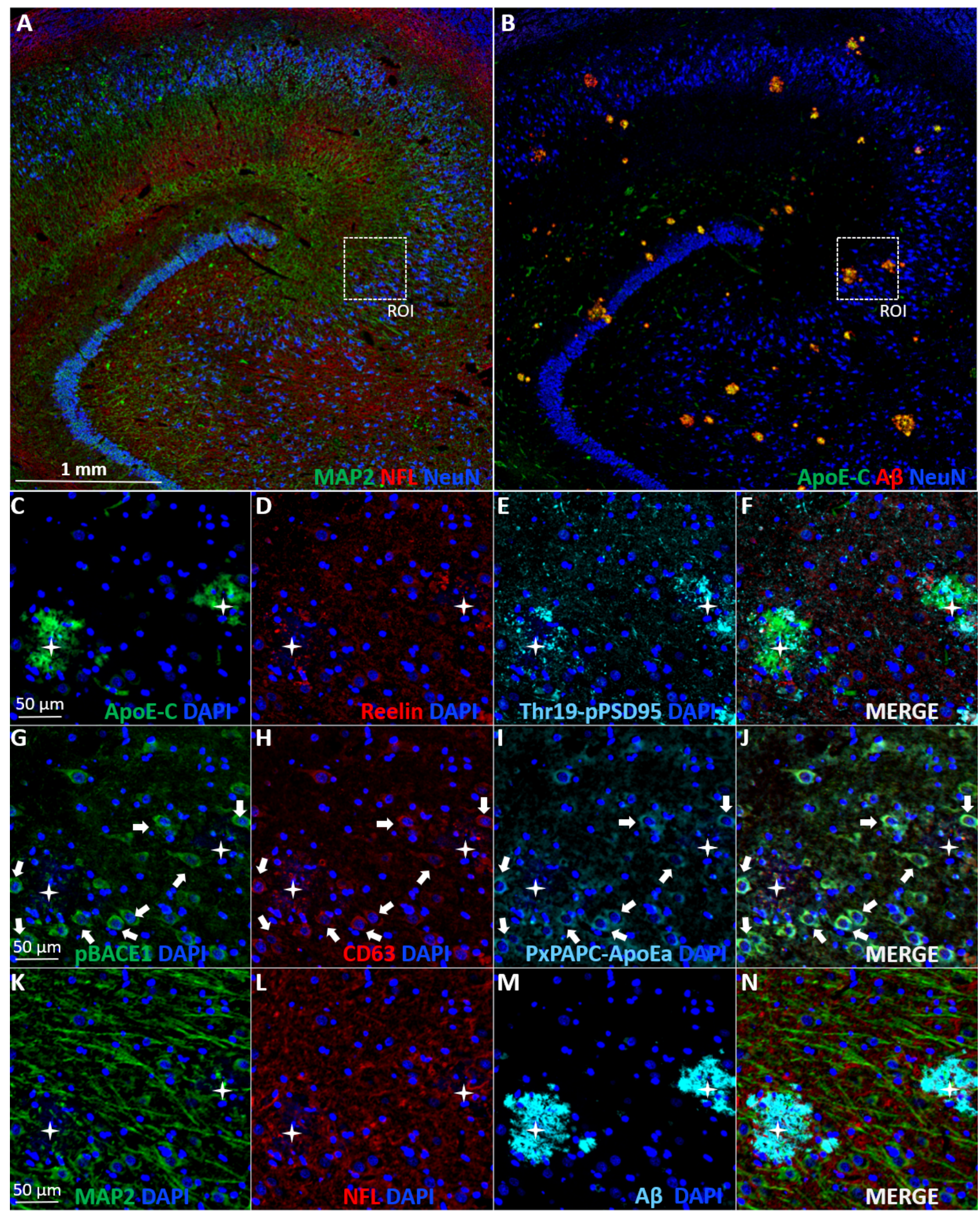


medRxiv preprint doi: https://doi.org/10.1101/2021.07.05.21259649; this version posted July 6, 2021. The copyright holder for this preprint (which was not certified by peer review) is the author/funder, who has granted medRxiv a license to display the preprint in perpetuity. This article is a US Government work. It is not subject to copyright under 17 USC 105 and is also made available for use under a CCO license.

Fig 9: ApoE/Reelin-ApoER2 axis derangements intersect with classic A $\beta$ related pathologies Multiplex IHC was used to stain a medial temporal lobe section from a representative case with advanced (Braak Stage V, ApoE3/3) AD with markers for the ApoE/Reelin cascade, classic histopathological biomarkers of $\mathrm{AD}$ and cytoarchitectural markers labeling neurons $(\mathrm{NeuN})(\mathbf{A}, \mathbf{B})$, dendrites (MAP2) (A, K, N), and axons (NFL) (A, L, N). One ROI (A, B) from the CA3 region of the hippocampus containing two neuritic plaques was enlarged in Panels $\mathbf{C}-\mathbf{N}$ to illustrate relationships between these markers. Immunoreactivities for antibodies targeting the lipid cargo transporting domain of ApoE (ApoE-C) (C), Reelin C-terminal domain (Reelin) (D), and Thr19-pPSD95 (marker of synaptic disassembly) (E) are enriched in the immediate vicinity of neuritic plaques (depicted by white stars in $\mathbf{C}-\mathbf{N}$ ) (F, merged). Plaques are surrounded by neurons (depicted by white arrows in G-J) and dystrophic neurites that co-label for Ser498-pBACE1 (G), CD63 (marker for late endosomes) (H), and lipid peroxidation-modified ApoE (PxPAPC-ApoEa) (I), consistent with intersection of these proteins in enlarged endolysosomal compartments in peri-plaque neurons. Tortuosity of MAP2 (K, N) and $\operatorname{NFL}(\mathbf{L}, \mathbf{N})$ immunolabeling is consistent with altered neuritic morphology in and around plaques.

These MP-IHC and single-marker IHC observations support the concept that the ApoE/Reelin-DAB1 axis intersects with $A \beta$ synthetic pathways within enlarged endo-lysosomal compartments in peri-plaque neurons and neurites. However, future studies with higher-resolution imaging are needed to identify the subcellular localizations of each of these molecular players. 
medRxiv preprint doi: https://doi.org/10.1101/2021.07.05.21259649; this version posted July 6, 2021. The copyright holder for this preprint

(which was not certified by peer review) is the author/funder, who has granted medRxiv a license to display the preprint in perpetuity. This article is a US Government work. It is not subject to copyright under 17 USC 105 and is also made available

\section{Discussion} for use under a CCO license.

\section{Overview of study findings}

The present studies were motivated by the hypothesis that the convergence of reactive sites within synaptic ApoE receptors and their ligands (Fig 1) creates a microenvironment that is vulnerable to lipid peroxidation, and that resulting downstream disruptions in ApoE delivery and Reelin signaling cascades contribute to pathogenesis of sporadic AD. Using a combination of biochemical experiments, single-marker IHC and MP-IHC to examine this hypothesis we found that: (1) double-Lys and His-enriched sequences within ApoER2 and ApoE, and recombinant ApoER2 and ApoE proteins, are susceptible to attack by reactive lipid aldehydes, generating lipid-protein adducts and acid-stable ApoER2-ApoE complexes (Figs 2, S3-S6); (2) ApoER2, ApoE, phospholipid peroxidation-modified ApoE, lipid aldehyde-modified ApoE, Reelin, and multiple downstream components of Reelin signaling cascades that govern cytoskeletal and synaptic integrity [including DAB1, Tyr607-pPI3K, Thr508-pLIMK1, Ser202/Thr205-pTau and Thr19-pPSD95] accumulate in the immediate vicinity of neuritic plaques and/or surrounding abnormal neurons (Figs 3-9, S13-35), and (3) several ApoE/Reelin-ApoE receptor-DAB1 pathway markers positively correlate with Braak stage, A $\beta$ plaque load, and antemortem cognitive impairment (Table 2 and Fig S2). These ApoE/Reelin-ApoER2-DAB1 axis pathologies were prominent in the dendritic compartments of the molecular layer of the dentate gyrus, cornu ammonis and subiculum, regions that receive synaptic input from the entorhinal-hippocampal projections that underlie memory formation. Taken together, these observations reveal extensive derangements in the ApoE/Reelin-ApoE receptor-DAB1 axis and provide evidence to support a new, working hypothesis (Fig 10) wherein aldehydic adduction and crosslinking of synaptic ApoE receptors and ApoE are proximate molecular events that compromise cytoskeletal and synaptic integrity, and contribute to the histopathological hallmarks and cognitive deficits that characterize sporadic AD in humans. 


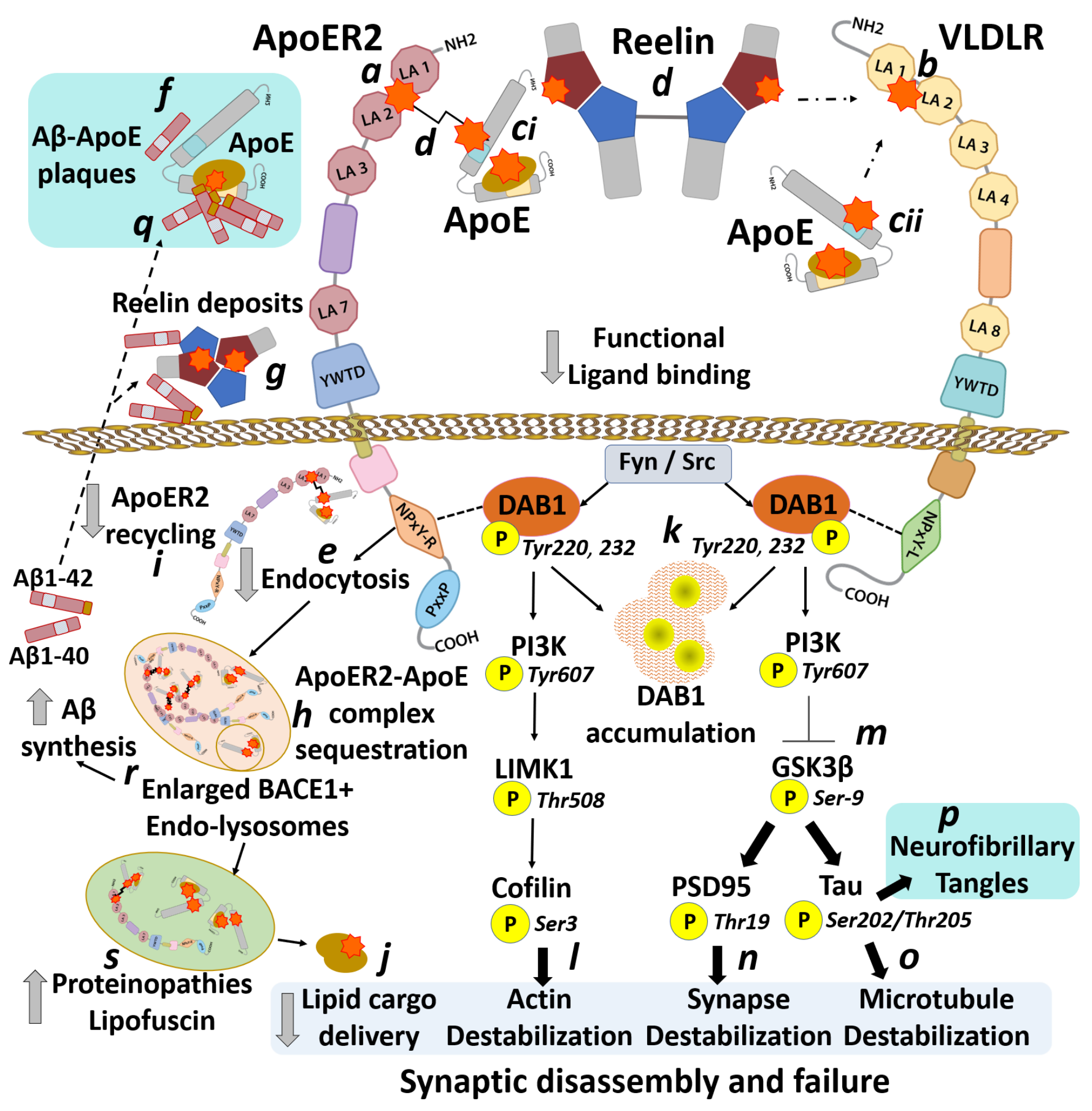


medRxiv preprint doi: https://doi.org/10.1101/2021.07.05.21259649; this version posted July 6, 2021. The copyright holder for this preprint

(which was not certified by peer review) is the author/funder, who has granted medRxiv a license to display the preprint in perpetuity. This article is a US Government work. It is not subject to copyright under 17 USC 105 and is also made available for use under a CCO license.

Fig 10: Working hypothesis: Proximate role of lipid peroxidation-induced ApoE/Reelin-ApoE receptorDAB1 axis disruption in Alzheimer's disease

Aldehydic products of lipid peroxidation derived from lipid-loaded ApoE particles attack Lys and His-enriched sequences within ApoER2 (a), VLDLR (b), and ApoE (ci-ii), to generate lipid-protein adducts and stable, crosslinked ApoE receptor-ApoE complexes (d). Adducts and crosslinks compromise functional ligand-ApoE receptor binding and impair internalization (e), leading to extracellular sequestration of ApoE (f) and Reelin (g). Acid-stable adducts and crosslinks disrupt ApoER2-ApoE complex dissociation in early endosomes (h), thereby disrupting ApoER2 recycling (i), and the delivery of the lipid cargo needed to remodel synaptic membranes (j). Impaired Reelin binding to ApoE receptors disrupts phosphorylation and proteasomal degradation of DAB1, and lysosomal degradation of ApoER2, leading to localized accumulation of DAB1 (k) and ApoER2 (see h). Ensuing compromise of the ApoER2-PI3K-LIMK1-Cofilin arm of the Reelin cascade destabilizes the actin cytoskeleton (I). Compromise of the VLDLR-PI3K arm of the Reelin cascade activates GSK3 $\beta$ by decreasing its phosphorylation (thick inhibition sign) (m). GSK3 $\beta$-mediated Thr19-phosphorylation of PSD95 (thick arrow) and Ser202/Thr205-phosphorylation of Tau (thick arrow) destabilize postsynaptic receptor complexes (n) and the microtubule cytoskeleton (o), respectively. GSK3 $\beta$-mediated Tau hyperphosphorylation also promotes the formation of neurofibrillary tangles (p). Extracellular ApoE deposition (see $\mathbf{f}$ ) serves as a nidus for oligomerization and deposition of $A \beta$, leading to $A \beta-A p o E$ plaque formation $(\mathbf{q})$. Sequestration of acid-stable ApoER2-ApoE complexes disrupts endosomal trafficking leading to enlarged endo-lysosomes with A $\beta P P$ and BACE1 colocalization, and enhanced A $\beta$ synthesis (r). Decreased lysosomal degradation of crosslinked lipidprotein polymers exacerbates proteinopathies and lipofuscin accumulation (s). Collective derangements are therefore proposed to help account for our study findings, as well the synaptic failure, and defining hallmarks of $\mathrm{AD}$ (aqua shading in $\mathbf{p}, \mathbf{q})$.

\section{ApoE in sporadic $A D$}

The discovery that inheritance of the ApoE4 allele is the most important genetic risk factor for sporadic AD was made in $1993 .{ }^{9-11}$ The ApoE2 allele was later shown to be protective, ${ }^{83}$ however the most salient mechanisms mediating the link between ApoE and AD remain elusive. ApoE is enriched in the core of many A $\beta$-protein deposits. ${ }^{61} 84$ In 2005, Thal et al ${ }^{85}$ observed that ApoE co-localizes with newly formed A $\beta$-protein deposits, implying a role for ApoE in the earliest stages of plaque formation. Unlike A $\beta$, virtually all ApoE immunoreactive plaques are associated with dystrophic neurites, ${ }^{61}{ }^{86}$ suggesting functional links between localized ApoE accumulation, neurite degeneration, and formation of the neuritic type of plaques that are most closely correlated with cognitive deficits. ${ }^{87} 88$ In the present study, we used MP-IHC to confirm that ApoE is enriched in the core of neuritic plaques (Figs 3-7, S11-12), is associated with antemortem cognitive impairment, and accumulates in accordance with AD progression (Table 2 and Fig S2). Since neurons obtain ApoE particles via receptor-mediated internalization, one potential explanation for the enrichment of ApoE in neuritic and newly formed plaques is impaired binding to ApoE receptors, leading to extracellular trapping of ApoE and its lipid cargo, where it could plausibly serve as a nidus for $A \beta$ oligomerization (Fig 10). ApoER2 and VLDLR are of particular interest in this respect because they are localized to synaptic membranes, ${ }^{23242689}$ can internalize ApoE particles, ${ }^{22} 4390$ and sit atop intraneuronal signaling (kinase) cascades that govern synaptic integrity and function. ${ }^{16-21}$ 
medRxiv preprint doi: https://doi.org/10.1101/2021.07.05.21259649; this version posted July 6, 2021. The copyright holder for this preprint

(which was not certified by peer review) is the author/funder, who has granted medRxiv a license to display the preprint in perpetuity. This article is a US Government work. It is not subject to copyright under 17 USC 105 and is also made available for use under a CCO license.

ApoE receptor 2 disruption: a pathological nexus in sporadic Alzheimer's disease?

Three years after the link between the ApoE4 allele and sporadic AD was reported, Kim et $\mathrm{al}^{22}$ discovered an ApoE-binding receptor (ApoER2) with high expression in the human brain. ApoER2 has multiple splice variants, ${ }^{56}$ including a proline-rich motif in the cytoplasmic tail that anchors ApoER2 to lipid rafts, ${ }^{23} 24$ enabling formation of multiprotein ApoER2-PSD95-NMDA receptor complexes 265556 within postsynaptic terminals of excitatory synapses. In rodent and cell models, ApoER2 plays pivotal roles in dendritic arborization, long-term potentiation, and memory. ${ }^{21}$ 25-27 91-95 However, our understanding of the role of ApoER2 in human AD is limited, in part due to lack of ApoER2 antibodies validated for IHC in human brain. To our knowledge, a 1999 report in which Motoi et $\mathrm{al}^{96}$ provides the only published ApoER2 IHC data for human AD brains. Motoi et al. reported increased immunolabeling of an ApoER2 cytoplasmic domain in hippocampal neurons in $\mathrm{AD}$ cases compared to non-AD controls.

To expand our understanding of ApoER2 in human AD, we raised an immunoprobe against the same double-Lys and His-enriched sequence ( $\mathrm{LDH}_{75} \mathrm{SDEDDCPK}_{83} \mathrm{~K}_{84} \mathrm{~T}$, ApoER2 LA1-2) within the ApoE and Reelin binding region that we previously found to be vulnerable to in vitro adduction and crosslinking (Figs 2 , S1-4). Using single-marker IHC and MP-IHC with multidomain labeling, we observed prominent ApoER2 LA1-2 immunoreactivity in the immediate vicinity of ApoE-enriched neuritic plaques in the entorhinal cortex, hippocampus, and especially the molecular layer of the dentate gyrus (Figs 3-5, S13-14). Enrichment of ApoER2 within neuritic plaques in the molecular layer of the dentate gyrus is noteworthy given that the dendritic arbors within this region are the major synaptic recipients of entorhinal-hippocampal projections ${ }^{57-59}$ that are implicated in the memory deficits of AD. ${ }^{67} 98$ Scheff et al. observed marked decreases in synaptic density and width ${ }^{34}$ of the molecular layer of the dentate gyrus in early AD. In adult ferrets, entorhinal cortex Layer II neurons and their perforant pathway projections and terminal arborization fields are heavily immunoreactive for Reelin, suggesting a role for anterograde transport and secretion of Reelin from axon terminals into the molecular layer of the dentate gyrus, ${ }^{99-101}$ where it could interact with ApoER2-enriched dendritic arbors emanating from dentate granule cells and pyramidal basket cells. ${ }^{102}$ Taken together, these observations point toward a central role for the Reelin-ApoER2 ligand-receptor pair in memory and raise the prospect that disruption of the Reelin/ApoE-ApoER2-DAB1 axis in this region could contribute to cognitive deficits in AD.

\section{ApoE and ApoER2 are vulnerable to peroxidation, adduction, and crosslinking}

A marked increase in lipid peroxidation is present in the earliest stages of AD. ${ }^{31-38 ~ 103-105}$ Since the human brain is enriched in polyunsaturated phospholipids that are vulnerable to peroxidation, ${ }^{52} 106107$ and ApoE is the main apolipoprotein that traffics these phospholipids to neurons, ${ }^{13-15}$ it is not surprising that ApoE and its lipid cargo are susceptible to peroxidation-related modifications. ${ }^{108-111}$ However, specific mechanisms linking peroxidation of lipids to AD pathogenesis remain elusive. The studies reported here were motivated by the 
medRxiv preprint doi: https://doi.org/10.1101/2021.07.05.21259649; this version posted July 6, 2021. The copyright holder for this preprint

(which was not certified by peer review) is the author/funder, who has granted medRxiv a license to display the preprint in perpetuity. This article is a US Government work. It is not subject to copyright under 17 USC 105 and is also made available for use under a CCO license.

hypothesis that the binding regions of synaptic ApoE receptors and their ligands are consequential targets of lipid peroxidation, and that their aldehydic adduction and crosslinking could disrupt ApoE-mediated delivery of lipids and Reelin-ApoE receptor signaling cascades (Figs 1, 10). Consistent with this hypothesis, we observed that ApoER2 $\left(\mathrm{LDH}_{75} \mathrm{SDEDDCPK}_{83} \mathrm{~K}_{84} \mathrm{~T}\right)$ and $\mathrm{ApoE}\left(\mathrm{ASH}_{140} \mathrm{LRK}_{143} \mathrm{LRK}_{146} \mathrm{~L}\right)$ peptides, and recombinant ApoER2 and ApoE proteins, are susceptible to attack by reactive lipid aldehydes, generating lipid-protein adducts and stable ApoER2-ApoE complexes. Peptide crosslinking was dependent upon both the His $\left(\mathrm{H}_{140}\right)$ residue and double-Lys motif $\left(\mathrm{K}_{143} \mathrm{LRK}_{146}\right)$ of ApoE and either the double-Lys motif $\left(\mathrm{K}_{83} \mathrm{~K}_{84}\right)$ or the His $\left(\mathrm{H}_{75}\right)$ residue of ApoER2. Since this double-Lys motif and His residue of human ApoER2 are absent in mice and rats, these vulnerabilities are likely unique to humans.

While the enrichment of native ApoE in the core of neuritic plaques is well-established, to our knowledge it is not yet known whether peroxidation modified ApoE is present in the plaque environment. We observed that immunoprobes targeting aldehyde-modified ApoE (CRA-ApoE and HNE-ApoE) strongly labeled small puncta near the central core of neuritic plaques, with nominal overlap with native ApoE labeling (Figs 5, 7, S19-21). By contrast, PxPAPC-ApoE immunoreactivity was minimal in plaques but prominent within a subset of adjacent neurons, especially those that co-expressed markers indicative of Reelin cascade dysfunction and synapse disassembly (Figs 5-6, 9, S18). Moreover, HNE-ApoE immunoreactivity positively correlated with neuritic plaque density and PxPAPC-ApoE immunoreactivity positively correlated with Braak stage and antemortem cognitive impairment. Taken together, these observations suggest that a portion of ApoE present in the plaque core and surrounding neurons has been modified by lipid peroxidation and provide a key pillar of support for our working hypothesis linking ApoE peroxidation to AD pathogenesis (Fig 10).

\section{Disruptions in the Reelin-ApoE receptor-DAB1 axis in sporadic AD}

In 1995, D'Arcangelo et al ${ }^{112}$ discovered that the cortical lamination defects in 'reeler' mice are due to mutations in the gene coding for the Reelin. While ensuing work on Reelin focused on its role in brain development, it is now clear that Reelin has essential functions in the mature brain (reviewed in ${ }^{21}$ ). In rodent and cellular models, Reelin increases the density, morphological complexity, and stability of post-synaptic elements of adult hippocampal synapses 161720232527 and plays central roles in learning and memory 1821262829 113 (Fig 1). These effects require binding of the Reelin double-Lys motif to the LA repeat 1 regions of ApoER2 and VLDLR 4142446 leading to phosphorylation and activation of DAB1, which in turn activates parallel kinase cascades that stabilize the actin and microtubule cytoskeletons (Fig 1). Findings from experimental models suggest that impairment of these Reelin-ApoE receptor-DAB1 cascades could contribute to AD (reviewed in ${ }^{114}$ ). Most notably, $A \beta$ impairs the binding of Reelin to ApoER2, thereby inhibiting DAB1 phosphorylation and abolishing Reelin-induced inhibition of Tau phosphorylation. ${ }^{71} 72$ Reelin extracted from frontal cortex of human $\mathrm{AD}$ cases immunoprecipitates with oligomeric $\mathrm{A} \beta$ species, ${ }^{72}$ suggesting that $\mathrm{A} \beta$ oligomers may disrupt the Reelin-ApoER2-DAB1 cascade by sequestering Reelin in the extracellular space. Despite these suggestive 
medRxiv preprint doi: https://doi.org/10.1101/2021.07.05.21259649; this version posted July 6, 2021. The copyright holder for this preprint

(which was not certified by peer review) is the author/funder, who has granted medRxiv a license to display the preprint in perpetuity. This article is a US Government work. It is not subject to copyright under 17 USC 105 and is also made available for use under a CCO license.

findings, evidence linking Reelin to AD in humans is conflicting. ${ }^{6} 68115116$ Reelin is secreted as a dimer ${ }^{117}$ and undergoes extensive cleavage (reviewed in ${ }^{118}$ ). Since commonly used Reelin antibodies target regions impacted by dimerization and outside of the diffusible, central fragment that harbors the ApoER2 and VLDLR binding motif, conflicting findings may be due in part to use of different antibodies. Our multidomain labeling approach (Fig S1), which included a novel immunoprobe targeting the Reelin binding motif, revealed enrichment of Reelin and its intracellular adapter DAB1 in the immediate vicinity of ApoE-enriched neuritic plaques (Figs 47, 9, S23-26), including those containing ApoER2-expressing dystrophic neurites. These observations, together with in vitro finding that Reelin central fragment forms lipid aldehyde-induced, crosslinked ApoER2-Reelin complexes in a similar manner as observed ApoE, provides an additional, interrelated mechanism in which lipid peroxidation could disrupt the ApoE/Reelin-ApoER2-DAB1 axis.

\section{Evidence for a pathological nexus: concurrent accumulation of Reelin, ApoE, ApoER2 and DAB1}

Reelin-ApoER2 signaling induces lysosomal degradation of ApoER2, ${ }^{23}$ while Reelin signaling through VLDLR induces degradation of Reelin itself. ${ }^{23}$ Reelin signaling through either ApoER2 and VLDLR induces proteasomal degradation of DAB1. ${ }^{60}$ Thus, our findings of extracellular accumulation of Reelin in the immediate vicinity of ApoER2- and DAB1-enriched dystrophic neurites implies a localized, functional deficit in Reelin signaling through ApoE receptors, rather than depletion of Reelin protein. Moreover, the extraneuronal accumulation of native, and lipid aldehyde modified ApoE in the core of neuritic plaques implies localized disruption of ApoE internalization. These observations, when considered together with our in vitro findings that ApoER2, ApoE, Reelin and VLDLR are vulnerable to lipid peroxidation, are consistent with the putative pathological nexus and working model (Fig 10), wherein lipid peroxidation disrupts the ApoE/ReelinApoE receptor-DAB1 axis, which disrupts the integrity of actin and microtubule cytoskeletons, and postsynaptic receptor clusters.

\section{Pathological disruption of the Reelin-ApoER2-DAB1-PI3K-LIMK1 actin stabilization pathway}

Dendritic spines are dynamic actin-rich protrusions that harbor excitatory synapses within postsynaptic densities. ${ }^{119}$ In rodent and cellular models, binding of Reelin to ApoER2 remodels and stabilizes the actin cytoskeleton of spines by evoking a kinase cascade involving Tyr220/232-pDAB1, Tyr607-pPI3K and Thr508pLIMK1, and culminating with Ser3-phosphorylation and inactivation of Cofilin ${ }^{74}$ 120-123 (Fig 1). Despite suggestions from experimental models, there is limited human IHC data supporting a role for this pathway in AD. Using MP-IHC to label each core component of this pathway, we observed strong immunoreactivities for Tyr607-pPI3K and Thr508-pLIMK1 in neuritic plaques, and for Tyr232-pDAB-1, Tyr607-pPI3K, Thr508pLIMK1, and Ser3-pCofilin in a subset of neurons adjacent to plaques (Figs 5-6, 8, S27-30). Moreover, Thr508-pLIMK1 accumulation in entorhinal cortex and hippocampus correlated with Braak Stage and brain A $\beta$ plaque load. Heredia et al ${ }^{124}$ previously observed a significant increase in the number of Thr508-pLIMK1 
medRxiv preprint doi: https://doi.org/10.1101/2021.07.05.21259649; this version posted July 6, 2021. The copyright holder for this preprint

(which was not certified by peer review) is the author/funder, who has granted medRxiv a license to display the preprint in perpetuity. This article is a US Government work. It is not subject to copyright under 17 USC 105 and is also made available for use under a CCO license.

positive neurons in $\mathrm{AD}$-affected regions, including some neurons that were devoid of classic AD pathologies. To our knowledge, Tyr607-pPI3K has not previously been shown to accumulate in neuritic plaques. Since Reelin signaling through ApoER2 induces PI3K and LIMK1 phosphorylation (Fig 1), accumulation of Tyr607pPI3K and Thr508-pLIMK1 in close proximity to Reelin and ApoE-enriched plaques provides additional evidence supporting Reelin-ApoER2-PI3K-LIMK1 cascade disruption and the putative pathological nexus shown in Fig 10.

\section{Pathological disruption of the Reelin-ApoE receptor-DAB1-PI3K-GSK3 $\beta$-Tau/PSD95 axis}

Local remodeling and stabilization of the microtubule cytoskeleton is also required to shape and strengthen synapses (reviewed in ${ }^{21}$ ). In rodent and cellular models, Reelin-ApoE receptor-DAB1 signaling stabilizes microtubules via a kinase cascade involving Tyr607-pPI3K-induced Ser9-phosphorylation and inhibition of GSK3 $\beta$, which decreases Tau phosphorylation (Fig 1). In rodent models, compromised Reelin signaling through ApoE receptors promotes GSK3 $\beta$-mediated Tau hyperphosphorylation and somatodendritic localization, ${ }^{66-68} 70$ and neurofibrillary tangle formation, which can be reversed by Reelin overexpression, ${ }^{125}$ implying a direct link between deficits in Reelin-ApoE receptor signaling and this defining AD pathology.

In rodent models, activated GSK-3 $\beta$ also phosphorylates PSD95 at the Thr19 position to induce synapse disassembly and LTD, ${ }^{47}$ suggesting that like Tau, PSD95 may be a component of the Reelin-ApoE receptor-DAB1 pathway. In the present study, we observed striking immunoreactivity for Thr19-pPSD95 in MAP2-positive dystrophic dendrites in proximity to ApoE- and Reelin-enriched plaques and adjacent neurons in regions affected by AD (Figs 5-9, S31-32), and strong positive correlations between Thr19-pPSD95 and Braak Stage, A $\beta$ plaque load, and especially ante-mortem cognitive impairment (Table 2). To our knowledge this is the first demonstration of Thr19-pPSD95 immunolabeling in human entorhinal cortex or hippocampus, and the first evidence linking Thr19-pPSD95 to AD pathology. These observations suggest that compromised Reelin-ApoE receptor-DAB1-PI3K signaling could enhance GSK3 $\beta$-mediated phosphorylation of both Tau (Ser 202/Thr205) and PSD95 (Thr19), leading to parallel destabilization of the microtubule cytoskeleton and postsynaptic receptor clusters within excitatory glutamatergic synapses, respectively. Anticipated functional correlates of such alterations may include enhanced LTD and synaptic dysfunction, while long-term pathological correlates may include synaptic loss with accumulation of Ser 202/Thr205-pTau and Thr19pPSD95 in the vicinity of neuritic plaques, as observed in this study. Since synapse dysfunction and loss are strong neurobiological correlates of cognitive deficits in AD, ${ }^{3} 126$ Thr19-phosphorylation of PSD95 should be studied further as both a potential underlying mechanism and as a biomarker for AD.

While the best-known physiological role of Tau is stabilization of microtubules within terminal axons, accumulating evidence indicates that Tau has crucial physiological functions in dendrites. ${ }^{127} 128$ Dendritic Tau interacts directly with PSD95 ${ }^{129}$ and is required to target Fyn to the post-synapse, where Fyn 
medRxiv preprint doi: https://doi.org/10.1101/2021.07.05.21259649; this version posted July 6, 2021. The copyright holder for this preprint

(which was not certified by peer review) is the author/funder, who has granted medRxiv a license to display the preprint in perpetuity. This article is a US Government work. It is not subject to copyright under 17 USC 105 and is also made available for use under a CCO license.

phosphorylates NMDA receptor subunit Glun2B 127130131 enabling formation of the PSD95-NMDAR complexes that mediate LTP. ${ }^{131}$ Intriguingly, in experimental models the neurotoxic effects of A $\beta$ are wholly dependent on dendritic Tau and are abolished by its deletion. ${ }^{127}$ Thr205-phosphorylation of Tau, which destabilizes microtubules, affords similar protection from A $\beta .{ }^{132} 133$ These observations, combined with evidence that the Reelin signaling cascades that are initiated upon Fyn phosphorylation of DAB1, ${ }^{134} 135$ are antagonized by extracellular $A \beta,{ }^{71} 72136$ imply physiological roles for $A \beta$ and Thr205-phosphorylation of Tau in the fine-tuning of Reelin-ApoER2-DAB1 mediated synaptic plasticity, and in protecting neurons from excitotoxicity. ${ }^{128}$ These findings raise the prospect that accumulations of extracellular $\mathrm{A} \beta$ and intraneuronal Thr205-pTau could reflect long-term, excessive, or misdirected protective responses, which ultimately manifest as the hallmark plaques and tangles that define AD.

The splice variant of ApoER2 that enables formation of postsynaptic ApoER2-PSD95-NMDA receptor complexes ${ }^{25} 2655$ helps account for the crucial role of ApoER2 in LTP and memory. ${ }^{55}{ }^{92-94}$ In the present study, we observed strong colocalization of Thr19pPSD95 and MAP2 (Fig 7), and localized enrichment of ApoER2, DAB1, Thr508-pLIMK1, Tyr607-pPI3K and Ser202/Thr205-pTau (Figs 4-9) in the vicinity of ApoE-, Reelinand $A \beta$-enriched neuritic plaques. These observations suggest that ApoER2 could serve as a molecular integrator, transmitting signals from extracellular Reelin, ApoE and $\mathrm{A} \beta$ to postsynaptic elements of excitatory synapses by regulating ApoER2-PSD95-NMDA-Tau complex integrity, Reelin-ApoER2-DAB1 signal transduction, and ultimately the balance between LTP and LTD. Moreover, we propose a model (Fig 10) in which ApoER2 disruption triggers a molecular switch from a synaptogenic to an amyloidogenic phenotype, with chronic disruption leading to hallmark AD pathologies including extracellular A $\beta$-ApoE complexes and intraneuronal neurofibrillary tangles. Future studies with high-resolution imaging may provide additional insights into subcellular localization of these markers, whereas in vitro knock out studies will be required to disentangle their complex functional interactions, and the sequence of molecular events governing synaptic integrity.

\section{ApoE receptors and endolysosomal pathway dysfunction}

Enlargement of neuronal endolysosomal compartments — which precedes A $\beta$ deposition ${ }^{137}$ —is among the earliest pathological findings in sporadic AD. ${ }^{78} 138$ Inheritance of the ApoE4 allele, but not genetic mutations that underlie familial AD, accentuates early endosome enlargement, indicating that altered endocytosis is not a consequence of $\mathrm{A} \beta$ deposition. ${ }^{137}$ Following receptor binding, ApoE-ApoE receptor complexes are internalized and trafficked to the early endosome, where the acidic environment (pH6) facilitates ligand release, ${ }^{43}$ allowing for swift recycling of ApoE receptors to the cell surface. ${ }^{139} 140$ In a series of elegant experiments by Herz et al, ${ }^{140}$ presence of ApoE4 impaired ApoER2 recycling, ${ }^{139}$ an effect that was reversed by pharmacological lowering of endosomal $\mathrm{pH} .{ }^{140}$ The authors attributed these observations to the propensity of ApoE4 to acquire a 'molten-globule' state near its isoelectric point at pH6.5, ${ }^{139} 140$ which in turn was proposed to interfere with 
medRxiv preprint doi: https://doi.org/10.1101/2021.07.05.21259649; this version posted July 6, 2021. The copyright holder for this preprint

(which was not certified by peer review) is the author/funder, who has granted medRxiv a license to display the preprint in perpetuity. This article is a US Government work. It is not subject to copyright under 17 USC 105 and is also made available for use under a CCO license.

ApoER2-ApoE dissociation and vesicle propagation through the endosomal recycling pathway. In the present study, we observed that ApoER2 and ApoE peptides and recombinant proteins are vulnerable to attack by reactive lipid aldehydes, generating acid-stable, crosslinked ApoER2-ApoE complexes and a wide variety of ApoER2 and ApoE adducts that were incompletely reversible in acidic conditions modeling the early endosome and lysosome (Fig 2, S3-6). These findings suggest that aldehydic modification and crosslinking of ApoER2 and ApoE could contribute to the compromised $\mathrm{pH}$-dependent dissociation and sequestration of ApoER2 and ApoE in endolysosomal compartments, impaired ApoER2 recycling, and ultimately to deficits in the ReelinApoER2-DAB1 signaling cascade and ApoE-mediated lipid delivery. In the present studies, ApoER2 enrichment, PxPAPC-ApoE accumulation, and endolysosomal enlargement were observed in the vicinity of ApoE-enriched plaques in both ApoE3/3 homozygote and ApoE3/4 heterozygote AD cases, implying that these pathologies are not dependent on the ApoE4-specific molten-globule state.

Moreover, the intraneuronal, granular staining pattern observed for PxPAPC-ApoE (Figs 5-6, 9, S18) suggests accumulation in one or more subcellular compartments. Given the physiological trafficking route of ApoE-containing lipid particles through the endolysosomal system, it is tempting to speculate that this granular pattern represents endolysosomal pathology. However, future studies with high-resolution imaging are needed to determine the specific subcellular localization of PxPAPC-ApoE.

\section{The ApoE/Reelin-ApoE receptor-DAB1 axis intersects with A $\beta$-related pathologies}

ApoER2 and A $\beta P P$ share intracellular adaptor proteins that regulate internalization and trafficking. ${ }^{141-144}$ In experimental models, the binding of ApoE and Reelin to ApoER2 regulates internalization of A $\beta P P$ and BACE1, the rate-limiting enzyme required for conversion of $A \beta P P$ to $A \beta .{ }^{141} 142$ These observations suggest that alterations in ApoE/Reelin-ApoER2-DAB1 signaling may regulate $A \beta$ production, however mechanisms linking lipoprotein receptors to AD are complex and incompletely understood (reviewed in ${ }^{145-148}$ ).

The neurotoxic effects of high $(\mu \mathrm{M})$ concentrations of $\mathrm{A} \beta$ are well-recognized and perhaps best exemplified by the aggressive course of familial $\mathrm{AD},{ }^{149}$ which is caused by genetic mutations that increase the synthesis or amyloidogenic processing of A $\beta$ PP. Although often overlooked, there is a substantial body of evidence indicating that $A \beta$ has protective functions at physiological $(<1 \mathrm{nM})$ concentrations. ${ }^{150-153}$ Moreover, unlike the genetic source of $\mathrm{A} \beta$ overproduction in familial AD (and transgenic mouse models), the most salient proximate trigger(s) for increased A $\beta$ synthesis and deposition in sporadic AD are unknown. Interestingly, BACE1 is activated by oxidative stress and other cellular stressors, ${ }^{154-158}$ implying a protective role for A $\beta$ against noxious stimuli. BACE1 is most active in acidic organelles. ${ }^{77}{ }^{159}$ In sporadic AD, altered endolysosomal trafficking leads to colocalization of $\mathrm{A} \beta \mathrm{PP}$ and $\mathrm{BACE} 1$ in late endosomes and lysosomes, facilitating $\mathrm{A} \beta$ synthesis and secretion. ${ }^{160}$ Intriguingly, at physiological concentrations, $A \beta$ acts as a potent antioxidant and metal chelator ${ }^{150-153}$ that is localized to lipoproteins, and appears to protect vulnerable polyunsaturated cargo from peroxidation. ${ }^{150} 161$ In in vitro models $A \beta$ interacts with the lipid cargo-transporting domain of ApoE. ${ }^{162}$ 
medRxiv preprint doi: https://doi.org/10.1101/2021.07.05.21259649; this version posted July 6, 2021. The copyright holder for this preprint

(which was not certified by peer review) is the author/funder, who has granted medRxiv a license to display the preprint in perpetuity. This article is a US Government work. It is not subject to copyright under 17 USC 105 and is also made available for use under a CCO license.

These collective observations led us to speculate that peroxidation-modified ApoE particles could serve as a noxious stimulus leading to endolysosomal compromise $(\mathbf{F i g} \mathbf{1 0 h}, \mathbf{t})$, and that ensuing BACE1 and A $\beta$ PP colocalization could initially serve a protective function by increasing neuronal secretion of $\mathrm{A} \beta$ monomers to bind and neutralize peroxidized ApoE particles (Fig 10s, r). However, with excessive or prolonged exposure, these protective effects of $A \beta$ could transition to the well-established neurotoxic effects of high $A \beta$ concentrations, overwhelming glial-mediated $\mathrm{A} \beta$ clearance pathways and leading to extracellular $\mathrm{A} \beta$ deposition (Fig 10f). This nuanced interpretation wherein BACE1 and A $\beta$ monomers initially serve a protective role is consistent with findings from controlled trials in which BACE1 inhibitors paradoxically worsened cognitive decline, ${ }^{163} 164$ despite markedly decreasing CSF A $\beta 1-40$ and A $\beta 1-42$ (Table S4). ${ }^{165}$

Consistent with these concepts, using MP-IHC we observed complex spatial and morphological relationships between $\mathrm{A} \beta$, Reelin, native ApoE, and lipid aldehyde-modified ApoE in plaques, and prominent cellular colocalization of Ser498-pBACE1, CD63, ApoER2, and PxPAPC-ApoE in surrounding neurons and dystrophic neurites (Figs 5-7, 9). Moreover, we observed extensive microglial infiltration and reactive astrocytosis (Figs 5-6, S10) in and around plaques that are enriched in A $\beta$ - and native and lipid peroxidationmodified ApoE, consistent with the known role of $A \beta$ in triggering microglia and astrocyte-mediated clearance pathways. ${ }^{166167}$ Since neurons obtain ApoE particles via receptor-mediated internalization, these MP-IHC observations, when considered together with evidence that ApoE (and especially oxidized ApoE) ${ }^{162}$ influence A $\beta$ oligomerization, ${ }^{162} 168169$ suggest that ApoE particles could plausibly become trapped in the synaptic cleft due to lipid peroxidation-induced ApoE receptor disruption, where they could serve as a nidus for $A \beta$ oligomerization, deposition and ultimately neuritic plaque formation (Fig 10f). This proposed sequence of events is consistent with evidence that in sporadic AD, ApoE is particularly enriched in newly formed ${ }^{85}$ and neuritic plaques ${ }^{61}$ and suggests that proximate mechanisms inducing plaque formation in sporadic AD (ApoE receptor disruption $\rightarrow$ localized ApoE deposition $\rightarrow \mathrm{A} \beta$-ApoE complex formation) may be distinct from those in familial $\mathrm{AD}$ and transgenic mouse models ( $\mathrm{A} \beta$ overproduction $\rightarrow \mathrm{A} \beta$ deposition). Future mechanistic studies are needed to determine if this proposed sequence of molecular events is valid. Future IHC studies comparing plaque contents of sporadic versus familial AD cases could shed further light on this topic.

\section{Unifying hypothesis and potential implications for therapeutics and prevention}

Sporadic AD is a major public health challenge that lacks effective treatments. The identification of proximate factors and a more complete understanding of molecular events underlying AD pathogenesis is needed and may lead to new and more effective therapeutics. ${ }^{170}$ The in vitro and IHC observations presented here provide preliminary evidence supporting a novel, plausible working hypothesis (Fig 10), wherein ApoE receptor and ApoE peroxidation, aldehydic adduction and crosslinking are proximate events that contribute to AD pathogenesis in humans by: (1) disrupting ApoE receptor-dependent delivery of lipid cargo required to remodel synaptic membranes; (2) trapping peroxidized ApoE particles outside neurons, where they serve as a 
medRxiv preprint doi: https://doi.org/10.1101/2021.07.05.21259649; this version posted July 6, 2021. The copyright holder for this preprint

(which was not certified by peer review) is the author/funder, who has granted medRxiv a license to display the preprint in perpetuity. This article is a US Government work. It is not subject to copyright under 17 USC 105 and is also made available for use under a CCO license.

nidus for $\mathrm{A} \beta$ oligomerization; (3) disrupting Reelin-ApoE receptor signaling cascades that stabilize actin and microtubule cytoskeletons and postsynaptic receptor complexes; (4) promoting Tau hyperphosphorylation and neurofibrillary tangle formation; (5) disrupting endolysosomal trafficking, which in turn; (6) increases $A \beta$ synthesis and exacerbates proteinopathies. This working model reframes synthesis and secretion of $A \beta$, and Thr205-phosphorylation of Tau as physiological responses involved in fine-tuning synaptic activity and protecting neurons from lipid peroxidation and excitotoxity that later contribute to the formation of plaques, tangles and neurodegeneration in AD. This unifying hypothesis could help account for the findings of the present study, as well as the histopathological hallmarks, loss of cytoskeletal and synaptic integrity, and cognitive deficits that characterize sporadic AD. The hypothesis is consistent with major genetic risk factors for sporadic $\mathrm{AD}$ - including variants in genes coding for ApoE, endolysosomal pathway components, and glial pathways that regulate phagocytosis and clearance of $\mathrm{A} \beta$, ApoE and lipids. The pathogenic nexus (Fig 1, 10) is also a convergence point for multiple established and suspected environmental risk factors for sporadic AD (Table S4). This working hypothesis is testable, and if experimentally corroborated, could have broad implications for the prevention and management of sporadic AD. Therapeutic implications and proposed pathogenic factors underpinning the PxApoE-ApoE receptor cascade versus the Amyloid Cascade hypothesis for sporadic AD are summarized in Table $\mathbf{S 4}$.

\section{Strengths \& limitations}

Well-recognized limitations of IHC applications include reduction or loss of less stable markers due to delayed, excessive or heterogenous tissue fixation, as well as non-specific antibody labeling. ${ }^{65} 171172$ To mitigate these limitations, we used only rapidly autopsied (Table 1) tissue specimens that underwent uniform fixation (employed by Brain and Body Donation Program at Banner Sun Health Research Institute). ${ }^{65}$ Moreover, we used IHC-validated antibodies whenever available and applied a multifaceted validation approach for new antibodies encompassing positive and negative controls, western blot, RNA-protein co-detection, conventional single-marker IHC, and MP-IHC with multidomain labeling. MP-IHC provided cytoarchitectural context, whereas multidomain labeling enabled use of multiple antibodies targeting different epitopes in the same proteins. While this combined approach increased confidence in the main study findings, it is still possible that postmortem tissue degradation, tissue processing abnormalities, or non-specific antibody labeling influenced results. Moreover, the time and resource-intensive nature of MP-IHC limited sample sizes. Future studies with more specimens are therefore needed to validate these results. Given the small sample sizes, statistical correlations of markers with Braak stage, $A \beta$ plaque load, and cognitive impairment should be considered preliminary. Future studies with more cases are needed to determine if these markers are influenced by ApoE genotype. Findings provide proof-of-concept that the specific Lys and His moieties within the ApoER2, ApoE, Reelin and VLDLR peptides examined in this study are vulnerable to aldehydic adduction and crosslinking. However, since each of these full-length proteins contain additional reactive amines, future studies are needed 
medRxiv preprint doi: https://doi.org/10.1101/2021.07.05.21259649; this version posted July 6, 2021. The copyright holder for this preprint (which was not certified by peer review) is the author/funder, who has granted medRxiv a license to display the preprint in perpetuity.

This article is a US Government work. It is not subject to copyright under 17 USC 105 and is also made available

to clarify the most relevant sites, types, and extent of lipid peroxidation-related modifications. Future higher magnification imaging is needed to determine the subcellular localizations of markers. Future studies are also needed to establish the sequence of molecular events leading to accumulation of markers investigated here, and whether such accumulations are a cause or consequence of AD.

\section{Summary and Conclusion}

The present results provide proof-of-concept that the synaptic ApoE receptors and their ligands are vulnerable to lipid peroxidation, aldehydic adduction and crosslinking, and demonstrate derangements in multiple ApoE/Reelin-ApoER2-DAB1 axis components associated with neuritic plaques, neurofibrillary tangles, and cognitive impairment in sporadic AD. Among these, Thr19-pPSD95 exhibited striking IHC staining and particularly strong positive correlations with histological progression and cognitive impairment. Collective findings provide the foundation for a working hypothesis implicating lipid peroxidation-related modifications of synaptic ApoE receptors and their ligands in the pathogenesis of sporadic AD in humans. 
medRxiv preprint doi: https://doi.org/10.1101/2021.07.05.21259649; this version posted July 6, 2021. The copyright holder for this preprint

(which was not certified by peer review) is the author/funder, who has granted medRxiv a license to display the preprint in perpetuity.

This article is a US Government work. It is not subject to copyright under 17 USC 105 and is also made available for use under a CCO license.

\section{Materials and Methods}

\section{Biochemical experiments}

Peptides, proteins, lipids, aldehydes, and other reagents were obtained as indicated in Table S1. Snakeskin dialysis tubing and Slide-A-lyzer dialysis units were obtained from Thermo Fisher. Spectra/Por dialysis apparatus and tubing were obtained from Repligen (Waltham, MA). Dialyses were performed using appropriate MWCO for protein or peptide molecular weights against 3 changes of the buffers specified below. KPhos refers to potassium phosphate buffer, pH 7.4 (Sigma, St. Louis, MO). 10mM PBS refers to 1x PBS, pH 7.4 (Quality Biological, Gaithersburg, MD).

\section{Antigen conjugate preparation}

Cu-oxidized PAPC-modified ApoE: In a vial, a chloroform solution of 6.24mg of PAPC (1-palmitoyl-2arachidonoyl-sn-glycero-3-phosphocholine) was evaporated to dryness under a stream of Argon. $15 \mathrm{ml}$ of $0.1 \mathrm{M}$ KPhos (pH7.4) containing 10mM CuSO 4 was added and it was vortexed. 2mg of ApoE4 monomer was added, it was vortexed again, and then incubated with gentle stirring at $37^{\circ} \mathrm{C}$ for 20 hours. The mixture was dialyzed extensively using snakeskin dialysis tubing (10kDa MWCO) against 0.1M KPhos+0.25 mM EDTA (pH7.4) (4L). The sample was lyophilized.

CRA-modified ApoE: A mixture of ApoE4 monomer (6mg) in 100mM crotonaldehyde in 10mM PBS (20ml) was incubated at room temperature for 3 days, then dialyzed against 10mM KPhos+0.25mM EDTA (4L).

HNE-modified ApoE: A solution of $25 \mathrm{mg}$ of 4-hydroxynonenal in $2 \mathrm{ml}$ EtOH was added to $15 \mathrm{ml}$ of $10 \mathrm{mM}$ PBS, turning cloudy. Then 5mg ApoE4 monomer was added, and it was mixed thoroughly. The mixture was incubated at room temperature for 4 days, then dialyzed against $10 \mathrm{mM}$ KPhos $(4 \mathrm{~L})$.

KOdiA-modified ApoE: A mixture of ApoE4 monomer (6mg) in 10mM PBS (20ml) containing 20mg of KOdiA-PC was incubated at room temperature for 3 days, then dialyzed against 10mM KPhos (4L).

ACET-modified ApoE: To a solution of 100mM acetaldehyde in 10mM PBS (20ml) was added 5.5mg of ApoE4 monomer. It was incubated at room temperature for 3 days and then a slight excess of $\mathrm{NaCNBH}_{3}(\sim 50 \mathrm{mg})$ was added and it was incubated overnight. Then the mixture was dialyzed against 10mM KPhos (4L).

\section{Peptide adduction and $\mathrm{pH}$ dependent reversibility experiments}

\section{Peptide Synthesis}


medRxiv preprint doi: https://doi.org/10.1101/2021.07.05.21259649; this version posted July 6, 2021. The copyright holder for this preprint

(which was not certified by peer review) is the author/funder, who has granted medRxiv a license to display the preprint in perpetuity.

This article is a US Government work. It is not subject to copyright under 17 USC 105 and is also made available for use under a CCO license.

Peptides were made by New England Peptide/Vivitide (Gardner, MA) using standard Fmoc solid-state peptide synthesis and purified using high-performance liquid chromatography (HPLC) to $>85 \%$ pure as determined by HPLC and/or LC/MS.

Peptide MDA adduction and pH dependent reversibility: 5mg of peptide (ApoER2 LA1-2 [Ac-

LDHSDEDD(C/Ac-C)PKKT-NH 2 , ApoE binding domain [Ac-ASHLRKLRKRL], VLDLR LA1-2 [AcVDGSDEKN(C/Ac-C)VKKT-NH ], or Reelin binding region [Ac-RFRWHQPAPFDKQQTW-NH ${ }_{2}$ ) was added to a solution of $1 \mathrm{mM}$ or $10 \mathrm{mM}$ MDA in $10 \mathrm{mM}$ PBS $(4 \mathrm{ml})$, and the mixture was incubated at $37^{\circ} \mathrm{C}$ for $48 \mathrm{hrs}$. Aliquots were removed for LC-MS analysis at $1 \mathrm{hr}, 3 \mathrm{hr}, 5 \mathrm{hr}, 24 \mathrm{hr}$, and $48 \mathrm{hr}$. At the end of the reaction, the mixture was dialyzed against 10mM PBS. For the ApoER2 LA1-2, ApoE binding domain, and VLDLR LA1-2 peptide adducts, approximately $1.5 \mathrm{ml}$ of the dialyzed solution was used to monitor the effect of acidic $\mathrm{pH}$ on the adduct stability, by incubating at $37^{\circ} \mathrm{C}$ in $10 \mathrm{mM}$ PBS adjusted with $0.1 \mathrm{M} \mathrm{HCl}$ to $\mathrm{pH} 6$ or $\mathrm{pH} 4$. Reactions were monitored by LC-MS over $48 \mathrm{hr}$ as above.

Reactive aldehyde mixture ApoER2 and ApoE peptide adduction and pH dependent reversibility: $5 \mathrm{mg}$ of the ApoER2 or ApoE binding domain peptide was added to a solution of $10 \mathrm{mM}$ PBS (4ml) containing $10 \mathrm{mM}$ each of MDA, acetaldehyde, CRA and 4-HNE and incubated at $37^{\circ} \mathrm{C}$ for $48 \mathrm{hrs}$. Aliquots were removed for LC-MS analysis at $3 \mathrm{hr}, 5 \mathrm{hr}, 24 \mathrm{hr}$, and $48 \mathrm{hr}$ and dialyzed against $10 \mathrm{mM}$ PBS. Approximately $1.5 \mathrm{ml}$ of the dialyzed solution was used to monitor the effect of acidic $\mathrm{pH}$ on the stability of the adducts, by incubating at $37^{\circ} \mathrm{C}$ at pH6 or pH4. Reactions were monitored by LC-MS over $48 \mathrm{hr}$ as above.

ApoER2 peptide analog (-Lys) MDA adduct: An analog of the ApoER2 LA1-2 peptide with sequence AcLDHSDEDD(C/Ac-C)PAAT-NH 2 (1mg) was incubated in $10 \mathrm{mM}$ MDA (10mM PBS; $1 \mathrm{ml})$ at $37^{\circ} \mathrm{C}$ for $48 \mathrm{hrs}$. Aliquots were removed for LC-MS analysis at 24hr and 48hr, dialyzed against 10mM PBS and analyzed by LCMS.

ApoE peptide analog (-Lys) MDA adduct: An analog of the ApoE binding domain peptide with sequence AcASHLRALRARL (1mg) was incubated in 10mM MDA (10mM PBS; 1ml) at $37^{\circ} \mathrm{C}$ for $48 \mathrm{hrs}$. Aliquots were removed for LC-MS analysis at $24 \mathrm{hr}$ and $48 \mathrm{hr}$, dialyzed against $10 \mathrm{mM}$ PBS and analyzed by LC-MS.

\section{Peptide crosslinking experiments}

ApoER2 LA1-2 + ApoE peptide 4-ONE crosslinking: A solution of 1mg (0.586 4 mol) of ApoER2 LA1-2 peptide and $0.83 \mathrm{mg}(0.586 \mu \mathrm{mol})$ of ApoE peptide was incubated in $10 \mathrm{mM}$ PBS $(586 \mu \mathrm{l})$ at $37^{\circ} \mathrm{C}$ for $20 \mathrm{~min}$. $109 \mu \mathrm{g}$ of 4 ONE $(0.702 \mu \mathrm{mol})$ in $21.8 \mu \mathrm{l}$ of EtOH was added, and it was incubated overnight. The mixture was analyzed by 
medRxiv preprint doi: https://doi.org/10.1101/2021.07.05.21259649; this version posted July 6, 2021. The copyright holder for this preprint (which was not certified by peer review) is the author/funder, who has granted medRxiv a license to display the preprint in perpetuity. This article is a US Government work. It is not subject to copyright under 17 USC 105 and is also made available for use under a CCO license.

LC-MS, and further characterized by TOF-MS after it was dialyzed in a $1 \mathrm{ml}$ Slide-A-lyzer against 10mM ammonium acetate $(200 \mathrm{ml})$.

ApoER2 LA1-2 peptide analog (-His) + ApoE peptide 4-ONE crosslinking: A solution of 1mg (0.610 $\mu \mathrm{mol})$ of ApoER2 peptide analog (-His) with sequence LDASDEDD(C/Ac-C)PKKT-NH 2 and $0.86 \mathrm{mg}(0.610 \mu \mathrm{mol})$ of ApoE binding region peptide was incubated in $10 \mathrm{mM}$ PBS $(610 \mu \mathrm{l})$ at $37^{\circ} \mathrm{C}$ for $20 \mathrm{~min} .113 \mu \mathrm{g}(0.732 \mu \mathrm{mol})$ of 4 ONE in 22.6 $\mu 1$ of EtOH was added, and it was incubated overnight, followed by analysis with LC-MS.

ApoER2 LA1-2 peptide + ApoE peptide analog (-Lys) 4-ONE crosslinking: A solution of 1mg (0.586 $\mu \mathrm{mol})$ of ApoER2 LA1-2 peptide and $0.76 \mathrm{mg}(0.586 \mu \mathrm{mol})$ of ApoE peptide analog with sequence Ac-ASHLRALRARL was incubated in $10 \mathrm{mM}$ PBS $(586 \mu \mathrm{l})$ at $37^{\circ} \mathrm{C}$ for $20 \mathrm{~min} .109 \mu \mathrm{g}(0.702 \mu \mathrm{mol})$ of $4-\mathrm{ONE}$ in $21.8 \mu \mathrm{lof} \mathrm{EtOH}$ was added, and it was incubated overnight, followed by analysis with LC-MS.

ApoER2 LA1-2 peptide + ApoE peptide analog (-His) 4-ONE crosslinking: A solution of 1mg (0.586 $\mu \mathrm{mol})$ of ApoER2 LA1-2 peptide and $0.794 \mathrm{mg}(0.586 \mu \mathrm{mol})$ of ApoE peptide analog with sequence AcASALRKLRKRL was incubated in $10 \mathrm{mM}$ PBS $(586 \mu \mathrm{l})$ at $37^{\circ} \mathrm{C}$ for $20 \mathrm{~min} .109 \mu \mathrm{g}(0.702 \mu \mathrm{mol})$ of $4-\mathrm{ONE}$ in $21.8 \mu \mathrm{l}$ of EtOH was added, and it was incubated overnight, followed by analysis with LC-MS.

ApoER2 LA1-2 peptide analog (-His) + ApoE peptide analog (-His) 4-ONE crosslinking: A solution of $1 \mathrm{mg}$ $(0.610 \mu \mathrm{mol})$ of ApoER2 LA1-2 peptide analog (-His) with sequence LDASDEDD(C/Ac-C)PKKT-NH 2 and $0.83 \mathrm{mg}(0.610 \mu \mathrm{mol})$ of ApoE peptide analog with sequence Ac-ASALRKLRKRL was incubated in 10mM PBS $(610 \mu \mathrm{l})$ at $37^{\circ} \mathrm{C}$ for $20 \mathrm{~min}$. $113 \mu \mathrm{g}(0.732 \mu \mathrm{mol})$ of $4-\mathrm{ONE}$ in $22.6 \mu \mathrm{l}$ of EtOH was added, and it was incubated overnight, followed by analysis with LC-MS.

ApoE peptide + ApoER2 LA1-2 peptide analog (-Lys) 4-ONE crosslinking: A solution of $1 \mathrm{mg}(0.628 \mu \mathrm{mol})$ of ApoER2 LA1-2 peptide analog (-Lys) with sequence LDHSDEDD(C/Ac-C)PAAT-NH 2 and 0.89mg $(0.628 \mu \mathrm{mol})$ of ApoE peptide was incubated in $10 \mathrm{mM}$ PBS $(628 \mu \mathrm{l})$ at $37^{\circ} \mathrm{C}$ for $20 \mathrm{~min} .116 \mu \mathrm{g}(0.754 \mu \mathrm{mol})$ of 4-ONE in $23.2 \mu \mathrm{l}$ of EtOH was added, and it was incubated overnight, followed by analysis with LC-MS.

ApoE peptide analog (-Lys) + ApoER2 LA1-2 peptide analog (-Lys) 4-ONE crosslinking: A solution of $1 \mathrm{mg}$ $(0.628 \mu \mathrm{mol})$ of ApoER2 LA1-2 peptide analog (-Lys) with sequence LDHSDEDD(C/Ac-C)PAAT-NH 2 and $0.82 \mathrm{mg}(0.628 \mu \mathrm{mol})$ of ApoE peptide analog with sequence Ac-ASHLRALRARL was incubated in 10mM PBS $(628 \mu \mathrm{l})$ at $37^{\circ} \mathrm{C}$ for $20 \mathrm{~min} .116 \mu \mathrm{g}(0.754 \mu \mathrm{mol})$ of $4-\mathrm{ONE}$ in $23.2 \mu \mathrm{l}$ of EtOH was added, and it was incubated overnight, followed by analysis with LC-MS. 
medRxiv preprint doi: https://doi.org/10.1101/2021.07.05.21259649; this version posted July 6, 2021. The copyright holder for this preprint (which was not certified by peer review) is the author/funder, who has granted medRxiv a license to display the preprint in perpetuity. This article is a US Government work. It is not subject to copyright under 17 USC 105 and is also made available

\section{Protein crosslinking experiments} for use under a CCO license.

ApoER2 + ApoE single aldehyde crosslinking: A solution of $100 \mu \mathrm{g}$ ApoER2 ectodomain and 100 $\mu \mathrm{g}$ of ApoE4 monomer in $10 \mathrm{mM}$ PBS $(343 \mu \mathrm{l})$ was incubated at $37^{\circ} \mathrm{C}$ for $10 \mathrm{~min}$, then one aldehyde (CRA, 4-ONE, 4-HNE, or MDA) was added to a final concentration of $10 \mathrm{mM}$ in $500 \mu 1$. After $48 \mathrm{hrs}$, the mixture was dialyzed against $10 \mathrm{mM}$ PBS.

ApoER2 + ApoE reactive aldehydes mixture crosslinking: A solution of $250 \mu \mathrm{g}$ of ApoER2 ectodomain and $250 \mu \mathrm{g}$ ApoE4 monomer in $10 \mathrm{mM}$ PBS $(607 \mu \mathrm{l})$ was incubated at $37^{\circ} \mathrm{C}$ for $10 \mathrm{~min}$, then a mixture of reactive aldehydes in PBS (consisting of 10mM each of MDA, CRA, acrolein, 4-HNE and 4-ONE) was added to a final concentration of $10 \mathrm{mM}$ per aldehyde in $1.25 \mathrm{ml}$. After $48 \mathrm{hrs}$, the mixture was dialyzed against $10 \mathrm{mM}$ PBS.

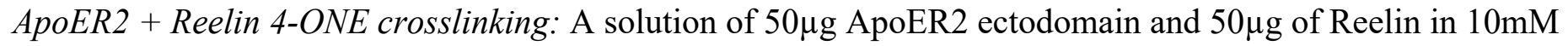
PBS $(181 \mu \mathrm{l})$ was incubated at $37^{\circ} \mathrm{C}$ for $10 \mathrm{~min}$, then $4-\mathrm{ONE}$ was added to a final concentration of $10 \mathrm{mM}$ in $241 \mu 1$. After $48 \mathrm{hrs}$, the mixture was dialyzed against $10 \mathrm{mM}$ PBS. 
medRxiv preprint doi: https://doi.org/10.1101/2021.07.05.21259649; this version posted July 6,2021 . The copyright holder for this preprint

(which was not certified by peer review) is the author/funder, who has granted medRxiv a license to display the preprint in perpetuity. This article is a US Government work. It is not subject to copyright under 17 USC 105 and is also made available for use under a CCO license.

\section{Liquid chromatography with mass spectrometry (LC-MS) and time-of-flight mass spectrometry (TOF-}

\section{MS) analysis of lipid-peptide adducts and crosslinked peptides}

HPLC experiments were performed on an Agilent 1100 LC system equipped with DAD detector and 6120 quadrupole mass spectrometer. The chromatographic separation was achieved on an Eclipse Plus C18 column (4.6mm x 50mm, $5 \mu \mathrm{m}$ ). The mobile phase consisted of $5 \%$ acetonitrile (aq) containing $0.2 \%$ formic acid (A) and $95 \%$ acetonitrile $(\mathrm{aq})$ containing $0.2 \%$ formic acid (B). The gradient elution program was as follows: $5 \% \mathrm{~B}$ from 0 to $3 \mathrm{~min}, 75 \% \mathrm{~B}$ from $3-12 \mathrm{~min}$, and $5 \% \mathrm{~B}$ at $12.01 \mathrm{~min}$, with a chromatographic runtime of $15 \mathrm{~min}$. The mobile phase flow rate was set at $0.4 \mathrm{~mL} / \mathrm{min}$ and the injection volume was $15 \mu 1$. MS scans were analyzed using ChemStation software (Agilent) with the electrospray ionization in positive mode and capillary voltage set at $4000 \mathrm{~V}$, nebulizer pressure at 45psig and gas flow at $12 \mathrm{~L} / \mathrm{min}$. The ApoER2 LA1-2 + ApoE peptide 4ONE crosslink experiment was characterized on a Triple $\mathrm{TOF}^{\mathrm{TM}} 5600+$ system with Duo-Spray ${ }^{\mathrm{TM}}$ ion source (Sciex, Framingham, MA). The sample was dissolved in $10 \mathrm{mM}$ ammonium acetate and directly infused using the on-board syringe pump at a flow rate of $10 \mu \mathrm{L} / \mathrm{min}$. The mass spectrometer was operating in the positive electrospray ionization (ESI) mode with the following settings: ion spray voltage, $5500 \mathrm{~V}$; the turbo spray temperature, $550^{\circ} \mathrm{C}$, declustering potential (DP), $60 \mathrm{~V}$ and collision energy (CE) $10 \mathrm{eV}$. Nebulizer gas (gas 1), heater gas (gas 2) and curtain gas were set to 60,60 and $20 \mathrm{~L} / \mathrm{min}$, respectively. MS scans were collected across various mass ranges between $m / z=500$ to 3800 and analyzed with Sciex OS and Analyst software (Sciex).

\section{Characterization of protein crosslinks and antibody specificity by Immunoblot}

Recombinant human ApoER2 ectodomain protein (R\&D, Cat\# 3520-AR-050) was reconstituted at 100 $\mu \mathrm{g} / \mathrm{mL}$ in sterile PBS. Recombinant human ApoE4 protein (Abcam, Cat\# ab50243) was reconstituted at $1 \mathrm{mg} / \mathrm{mL}$ in water. Recombinant human ApoER2 including C-terminal domain (Origene, TP320903) was supplied at $180 \mu \mathrm{g} / \mathrm{mL}$. Recombinant human VLDLR protein (Sigma, Cat\# SRP6469) was reconstituted at $50 \mu \mathrm{g} / \mathrm{mL}$ in water. All protein stocks were aliquoted and stored at $-80^{\circ} \mathrm{C}$. Recombinant proteins were diluted to $25 \mathrm{ng} / \mu \mathrm{L}$ in 1X Laemmli Buffer (Bio-Rad Laboratories, Inc., Cat\# 1610747) for SDS-PAGE analysis. Recombinant protein lipid aldehyde reaction mixtures were diluted to $50 \mathrm{ng} / \mu \mathrm{L}$ in $1 \mathrm{X}$ Laemmli Buffer and samples were boiled for 5 min at $100^{\circ} \mathrm{C}$. Ten $\mu \mathrm{L}$ from each sample were loaded onto 8-16\% Mini-PROTEAN TGX Precast Protein Gels (Bio-Rad Laboratories, Inc., Cat\# 4561106) and proteins were separated by electrophoresis according to the manufacturer's instructions. Gels were transferred to Immobilon-FL PVDF membrane (MilliporeSigma,Cat\# IPFL00010) for low background fluorescence western blotting. Total protein transfer efficiency was determined using AzureRed Fluorescent Total Protein Stain (Azure Biosystems, Inc., Cat\# AC2124) immediately after transfer following manufacturer's instructions with some modification. Membranes were stained for 20 min and washes were performed 3 times for $5 \mathrm{~min}$ with 100\% ethanol. Membranes were kept in dark storage containers protected from light until imaging. Stained membranes were immediately blocked with Azure Fluorescent Blot Blocking Buffer (Azure Biosystems, Inc., Cat\# AC2190) for 1 hour and primary antibodies were diluted in 
medRxiv preprint doi: https://doi.org/10.1101/2021.07.05.21259649; this version posted July 6, 2021. The copyright holder for this preprint (which was not certified by peer review) is the author/funder, who has granted medRxiv a license to display the preprint in perpetuity.

This article is a US Government work. It is not subject to copyright under 17 USC 105 and is also made available

fresh blocking buffer and incubated with each membrane overnight. Primary antibodies and dilutions used in these experiments are listed in Table S1. Multiplex fluorescent immunoblotting was accomplished by coincubating rat and mouse or rat and rabbit primary antibodies together in overnight incubations. The next day, membranes were washed $3 \mathrm{x}$ for 10 min using PBS containing 0.2\% Tween-20 (Sigma-Aldrich, Inc., Cat\# P9416). AzureSpectra fluorescent secondary antibodies were applied after washes at a 1:10,000 dilution in fluorescent blocking buffer and incubated for $1 \mathrm{hr}$. Membranes were washed 3x for $10 \mathrm{~min}$ using PBS containing $0.2 \%$ Tween-20, and $1 \mathrm{x}$ for 5 min using PBS. PVDF membranes were dried by placing on Kimwipes and stored in a drawer until completely dry. Blots were imaged using the smart scan function of the Sapphire Biomolecular Imager (Azure Biosystems, Inc.). 
medRxiv preprint doi: https://doi.org/10.1101/2021.07.05.21259649; this version posted July 6, 2021. The copyright holder for this preprint

(which was not certified by peer review) is the author/funder, who has granted medRxiv a license to display the preprint in perpetuity. This article is a US Government work. It is not subject to copyright under 17 USC 105 and is also made available for use under a CCO license.

\section{Immunohistochemistry}

\section{Postmortem specimens}

Rapidly autopsied medial temporal lobe specimens from 34 cases (Table 3) spanning the clinicopathological spectrum of $\mathrm{AD}$ were obtained from the Brain and Body Donation Program (BBDP) at the Banner Sun Health Research Institute (http://www.brainandbodydonationprogram.org). All BBDP subjects provided written consent for study procedures, autopsy and sharing of de-identified data prior to enrollment. The study and its consenting procedures were approved by the Western Institutional Review Board of Puyallup, Washington. The Banner BBDP population has been extensively described by Beach et al. ${ }^{65}$ 173-175 Briefly, most BBDP donors were enrolled as cognitively normal volunteers residing in the retirement communities near Phoenix, Arizona, USA. Following provision of informed consent, donors received standardized medical, neurological, and neuropsychological assessments during life. Ante-mortem cognitive testing battery included the Mini-Mental Status Exam (MMSE), The CDR Sum, FAST Score, Figure Recall Score, AVLT Total Learning, and AVLT A6. The MMSE Test had the least missing data and was the main cognitive endpoint of for the present study.

Neuropathological procedures and examinations performed by BBDP has been previously published. ${ }^{65}$ Briefly, the Braak neurofibrillary stage (0-VI) was determined using thick $40-80$-micron sections stained with Gallyas, Campbell-Switzer and thioflavine S stains as originally defined by Braak and Braak. ${ }^{63}$ Senile plaque density — including neuritic, cored, and diffuse plaques — was assessed in standard regions of the frontal, temporal, and parietal lobes, hippocampal CA1 region and entorhinal/transentorhinal region. Each region was assigned a semi-quantitative score of none, sparse, moderate and frequent and converted to numerical values 0 3, according to the CERAD templates. ${ }^{53}$ Plaque total is the arithmetic sum of scores from these five regions ranged from $0-15$. Neurofibrillary tangle density was assessed in the same five regions, with CERAD templates used to obtain semi-quantitative scores of none, sparse, moderate and frequent and these are converted to numerical values $0-3$. Tangle total is the arithmetic sum of scores from these five regions ranged from 0 - 15. The NIA-Reagan ${ }^{54}$ consensus recommendations were used for postmortem diagnosis of AD with high, intermediate and low referring to the likelihood that dementia, if present, is due to AD histopathology. AD was at a minimum defined as intermediate or high NIA-Reagan criteria. Mild Cognitive Impairment denoted the presence of this diagnosis at the time of death. A control designation is a participant without dementia or parkinsonism during life and without a major neuropathological diagnosis.

There is substantial literature suggesting that certain molecular components deteriorate after death, and the relationship with postmortem interval (PMI) and tissue quality is well-established. To mitigate the limitations of tissue deterioration and of excessive or variable fixation, the BBDP is dedicated to a short PMI and used standardized fixation procedures in which $1 \mathrm{~cm}$ sections were fixed in $10 \%$ formalin for 48 hours. For the present study, $6 \mu \mathrm{m}$-thick coronal medial temporal lobe paraffin sections at the level of the body of the 
medRxiv preprint doi: https://doi.org/10.1101/2021.07.05.21259649; this version posted July 6, 2021. The copyright holder for this preprint (which was not certified by peer review) is the author/funder, who has granted medRxiv a license to display the preprint in perpetuity.

This article is a US Government work. It is not subject to copyright under 17 USC 105 and is also made available

hippocampus were collected on Leica Apex Superior adhesive slides (VWR, 10015-146) to facilitate multiplex IHC. 
Table 3. Thirty-four cases spanning the clinicopathological spectrum of Alzheimer's disease progression

\begin{tabular}{|c|c|c|c|c|c|c|c|c|c|c|c|c|c|}
\hline ID & $\begin{array}{l}\text { Expired } \\
\text { age }\end{array}$ & Gender & $\begin{array}{l}\text { Post mortem } \\
\text { interval (hours) }\end{array}$ & $\begin{array}{c}\text { Braak } \\
\text { stage (0-6) }\end{array}$ & $\begin{array}{l}\text { MMSE } \\
(\mathbf{0 - 3 0})\end{array}$ & $\begin{array}{c}\text { Total } \\
\text { plaques }(0- \\
15)^{a} \\
\end{array}$ & $\begin{array}{c}\text { Total } \\
\text { tangles }(0- \\
15)^{\mathrm{a}} \\
\end{array}$ & $\begin{array}{l}\text { Neuritic plaque } \\
\text { density }(0-3)\end{array}$ & $\begin{array}{l}\text { ApoE } \\
\text { status }\end{array}$ & $\begin{array}{l}\text { Conventional } \\
\text { IHC b }\end{array}$ & $\begin{array}{c}\text { MP-IHC } \\
(\mathbf{n}=\mathbf{2 4})^{\mathrm{c}}\end{array}$ & $\begin{array}{l}\text { MP-IHC } \\
(n=12)^{d}\end{array}$ & $\begin{array}{c}\text { RNA-protein } \\
\text { Codetection }(n=12)\end{array}$ \\
\hline \multicolumn{14}{|c|}{ Alzheimer's Disease } \\
\hline 1 & $85-89$ & Female & 3.42 & Stage VI & 2 & 15 & 15 & 3 , frequent & $3 / 3$ & $\mathrm{X}$ & $\mathrm{X}$ & & \\
\hline 2 & $85-89$ & Female & 2.43 & Stage VI & 3 & 9 & 10 & 3 , frequent & $3 / 4$ & $\mathrm{X}$ & & & \\
\hline 3 & $55-59$ & Female & 1.83 & Stage VI & 3 & 15 & 15 & 3 , frequent & $3 / 3$ & $\mathrm{X}$ & & & \\
\hline 4 & $70-74$ & Male & 4.83 & Stage VI & 7 & 15 & 15 & 3 , frequent & $3 / 4$ & $\mathrm{X}$ & $\mathrm{X}$ & & \\
\hline 5 & $80-84$ & Female & 4 & Stage VI & 19 & 15 & 15 & 3 , frequent & $3 / 3$ & $\mathrm{X}$ & $\mathrm{X}$ & & \\
\hline 6 & $80-84$ & Female & 3.33 & Stage VI & 24 & 15 & 12 & 3 , frequent & $3 / 3$ & $\mathrm{X}$ & $\mathrm{X}$ & & \\
\hline 7 & $75-79$ & Female & 3.08 & Stage V & 6 & 15 & 15 & 3 , frequent & $3 / 3$ & $\mathrm{X}$ & $\mathrm{X}$ & $\mathrm{X}$ & $\mathrm{X}$ \\
\hline 9 & $80-84$ & Male & 4 & Stage V & 14 & 14 & 13 & 3 , frequent & $3 / 4$ & $\mathrm{X}$ & $\mathrm{X}$ & $\mathrm{X}$ & $\mathrm{X}$ \\
\hline 10 & $85-89$ & Male & 2.2 & Stage V & 21 & 13 & 11 & 3 , frequent & $2 / 3$ & $\mathrm{X}$ & $\mathrm{X}$ & & \\
\hline \multicolumn{14}{|c|}{ Mild Cognitive Impairment } \\
\hline 11 & $90+$ & Female & 3.16 & Stage IV & 22 & 5 & 9 & 3 , frequent & $3 / 3$ & $\mathrm{X}$ & & & \\
\hline 12 & $85-89$ & Male & 4.17 & Stage IV & 23 & 0 & 7 & 0, none & $3 / 3$ & $\mathrm{X}$ & $\mathrm{X}$ & & \\
\hline 13 & $80-84$ & Male & 2.67 & Stage IV & 24 & 0 & 9 & 0, none & $3 / 3$ & $\mathrm{X}$ & $\mathrm{X}$ & & \\
\hline 14 & $75-79$ & Female & 3.17 & Stage IV & 24 & 6 & 9 & 0, none & $2 / 3$ & $\mathrm{X}$ & $\mathrm{X}$ & $\mathrm{X}$ & $\mathrm{X}$ \\
\hline 15 & $90+$ & Female & 3.16 & Stage IV & 26 & 14 & 8 & 3 , frequent & $2 / 3$ & $\mathrm{X}$ & $\mathrm{X}$ & $\mathrm{X}$ & $\mathrm{X}$ \\
\hline 16 & $80-84$ & Female & 3 & Stage IV & 29 & 13 & 7 & 3 , frequent & $2 / 3$ & $\mathrm{X}$ & $\mathrm{X}$ & & \\
\hline 17 & $80-84$ & Female & 3.17 & Stage IV & 29 & 12 & 9 & 3 , frequent & $2 / 3$ & $\mathrm{X}$ & $\mathrm{X}$ & & \\
\hline 20 & $85-89$ & Male & 2.25 & Stage III & 28 & 9 & 6 & 2 , moderate & $3 / 3$ & $\mathrm{X}$ & $\mathrm{X}$ & $\mathrm{X}$ & $\mathrm{X}$ \\
\hline 21 & $90+$ & Male & 3 & Stage III & 28 & 1 & 4 & 1, sparse & $3 / 3$ & & & & $\mathrm{X}$ \\
\hline \multicolumn{14}{|c|}{ Age-matched Controls } \\
\hline 22 & $80-84$ & Male & 2.75 & Stage III & 25 & 13 & 9 & 1, sparse & $3 / 3$ & $\mathrm{X}$ & & & \\
\hline 23 & $90+$ & Female & 3 & Stage III & 27 & 7 & 5 & 1, sparse & $3 / 3$ & $\mathrm{X}$ & $\mathrm{X}$ & & \\
\hline 24 & $85-89$ & Female & 3.08 & Stage III & 28 & 0 & 6 & 0, none & $3 / 4$ & $\mathrm{X}$ & $\mathrm{X}$ & & \\
\hline 25 & $75-79$ & Female & 2.83 & Stage III & 29 & 10 & 5 & 2 , moderate & $3 / 3$ & $\mathrm{X}$ & $\mathrm{X}$ & & \\
\hline 26 & $75-79$ & Female & 2.5 & Stage II & 28 & 0 & 3 & 0, none & $3 / 3$ & $\mathrm{X}$ & $\mathrm{X}$ & $\mathrm{X}$ & $\mathrm{X}$ \\
\hline 27 & $75-79$ & Male & 4.3 & Stage II & 28 & 0 & 2 & 0, none & $2 / 3$ & $\mathrm{X}$ & $\mathrm{X}$ & & \\
\hline 28 & $75-79$ & Male & 3 & Stage II & 29 & 0 & 3 & 0, none & $3 / 3$ & $\mathrm{X}$ & $\mathrm{X}$ & $\mathrm{X}$ & \\
\hline 29 & $90+$ & Male & 3.41 & Stage I & 27 & 1 & 2 & 1, sparse & $3 / 3$ & $\mathrm{X}$ & $\mathrm{X}$ & $\mathrm{X}$ & $\mathrm{X}$ \\
\hline 30 & $75-79$ & Male & 2.33 & Stage I & 29 & 6 & 0 & 1, sparse & $3 / 4$ & $\mathrm{X}$ & $\mathrm{X}$ & & \\
\hline 31 & $85-89$ & Male & 3 & Stage I & & 6 & 2 & 1, sparse & $3 / 3$ & $\mathrm{X}$ & & & \\
\hline \multicolumn{14}{|c|}{ Young Control } \\
\hline 32 & $50-54$ & Female & 4.67 & Stage I & & 0 & 1 & 0, none & $3 / 3$ & & & $X$ & $\mathrm{X}$ \\
\hline 33 & $35-39$ & Male & 3 & Stage 0 & & 0 & 0 & 0, none & $3 / 3$ & & & $X$ & $\mathrm{X}$ \\
\hline 34 & $45-49$ & Male & 4.5 & Stage 0 & & 0 & 0 & 0, none & $3 / 3$ & & & $\mathrm{X}$ & $\mathrm{X}$ \\
\hline
\end{tabular}

a Total amyloid plaque density score in the following regions: frontal, temporal, parietal, hippocampus, and entorhinal cortex. Region scores: $0=$ none; $1=$ sparse; $2=$ moderate; $3=$ frequent.

${ }^{\mathrm{b}}$ Includes conventional IHC staining of AD cases and controls to determine optimal retrieval and staining parameters and/or use of MTL specimens for single-marker IHC throughout the spectrum of AD.

c Alzheimer's disease, Mild Cognitive Impairment, and Control cases included in age-matched MP-IHC.

d Specimens from Case 21 were substituted in place of 28 in a subset of rounds due to loss of specimens. 
medRxiv preprint doi: https://doi.org/10.1101/2021.07.05.21259649; this version posted July 6, 2021. The copyright holder for this preprint

(which was not certified by peer review) is the author/funder, who has granted medRxiv a license to display the preprint in perpetuity. This article is a US Government work. It is not subject to copyright under 17 USC 105 and is also made available

\section{Antibody selection and multidomain labeling approach}

To investigate key molecular components of the ApoE/Reelin-ApoER2-DAB1 axis and the hypothesized pathological nexus underlying AD (Figs 1 and 10), we applied a multidomain labeling strategy with 28 probes targeting different domains or epitopes within ApoER2, native and lipid-peroxidation modified ApoE, Reelin, DAB1, and downstream components of Reelin signaling cascades (Fig S1). The use of several antibodies targeting each protein, together with RNA-protein codetection, assisted in the validation of observations made using single antibodies, thereby adding confidence to our main study findings. To provide pathological and cytoarchitectural context, immunoprobes targeting classic AD pathological markers and cytoarchitectural markers were used alongside ApoE/Reelin-ApoER2-DAB1 axis antibodies. Several rabbit and chicken polyclonal antibodies and rat monoclonal antibodies were custom-generated, and affinity purified by New England Peptide/Vivitide and Precision Antibody, respectively (Table S1). The primary antibodies and dilutions are listed in Table $\mathbf{S 1 .}$

\section{Traditional single-marker IHC}

Single-marker IHC was used in the present studies to: (1) to determine optimal antibody concentrations and empirically optimize antibody-specific retrieval parameters in preparation for MP-IHC; (2) for negative controls (comparing staining results versus pre-immune serum and with the primary antibody omitted) and for positive controls (temporal or frontal cortex of AD cases versus healthy controls); and (3) to visualize and quantify markers throughout the spectrum of AD progression and as an additional strategy for validating key MP-IHC observations.

\section{Antibody controls}

Negative controls included substitution of pre-immune serum for primary antibodies and the use of secondaries with the primary antibody omitted (control for non-specific binding of the secondary antibody). Positive controls included staining of temporal and frontal cortex specimens from rapidly autopsied, neuropathologically characterized, confirmed AD cases (and young controls) with known region-specific A $\beta$ and tangle scores. These specimens were processed and fixed in an identical manner to the medial temporal lobe specimens.

\section{Preparation for MP-IHC}

AD plus young Control FFPE blocks were made by combining the temporal cortex and frontal cortex of rapidly autopsied brain specimens obtained from BBDP Banner Sun Health. Combined blocks were sectioned into 6 $\mu \mathrm{m}$-thick coronal sections, generating positive and negative control tissues in the same section. Using these sections, optimal immunostaining conditions were then empirically determined using an incremental retrieval paradigm including pH 6 10nM Na/Citrate pH6 and/or TRIS/EDTA pH 9 following parameters: $70^{\circ} \mathrm{C} / 10 \mathrm{~min}$, $70^{\circ} \mathrm{C} / 20 \mathrm{~min}, 70^{\circ} \mathrm{C} / 40 \mathrm{~min}$. Selected antibodies with suboptimal retrieval up to 40 minutes underwent 
medRxiv preprint doi: https://doi.org/10.1101/2021.07.05.21259649; this version posted July 6, 2021. The copyright holder for this preprint

(which was not certified by peer review) is the author/funder, who has granted medRxiv a license to display the preprint in perpetuity. This article is a US Government work. It is not subject to copyright under 17 USC 105 and is also made available for use under a CCO license.

additional retrieval rounds using formic acid (88\%, $20 \mathrm{~min}$ ) or proteinase $\mathrm{K}$ (Dako S3020) for $15 \mathrm{~min}$ at room temperature. Secondary antibodies used for single IHC chromogenic staining were appropriately matched to the host class/subclass of the primary antibody (Table S1).

\section{Multiplex Fluorescence Immunohistochemistry and In Situ Hybridization}

Multiplex fluorescence immunohistochemistry (MP-IHC) and multiplex fluorescence in situ hybridization (MPISH) were performed on $6 \mu$ m-thick coronal medial temporal lobe paraffin sections at the level of the body hippocampus, sourced from rapidly autopsied post-mortem brains provided by BBDP (Tables 1 and $\mathbf{3}$ ).

Specimens to be used for MP-IHC were sectioned onto Leica Apex Superior adhesive slides (VWR, 10015-146) to prevent loss of tissue. MP-IHC was carried out using up to 6 iterative rounds of sequential immunostaining with select antibody panels targeting the ApoE/Reelin-ApoE receptor-DAB1 axis and classical biomarkers of AD pathology, alongside classical cytoarchitectural biomarkers to map brain tissue parenchyma (see Table S1). To carry out iterative rounds of MP-IHC biomarker screening, the fluorophore conjugated targets from each round were imaged by multispectral epifluorescence microscopy followed by antibody stripping and tissue restaining steps to repeat the cycle, as previously described ${ }^{176}$ each time using a different antibody panel. MP-ISH was carried out on a subset of sections to screen for ApoER2 and Reelin expression mRNAs using custom designed RNA probes (Tables S1-2) and standard RNAscope Multiplex Fluorescent V2 hybridization kits (Advanced Cell Diagnostics, Inc.) per manufacturer's instructions. Sections probed with RNAscope were subsequently processed with 2 sequential rounds of MP-IHC to facilitate RNA-protein codetection. Briefly, for screening tissues by MP-IHC and/or MP-ISH, the sections were first deparaffinized using standard Xylene/Ethanol/Rehydration protocol followed by antigen unmasking with 10-15 minute heat mediated antigen retrieval step in $10 \mathrm{mM}$ Tris/EDTA buffer pH 9.0 (Tris/EDTA buffer) using a 800W microwave set at $100 \%$ power. Sections intended for MP-ISH were then processed using the RNAscope V2 kit (Advanced Cell Diagnostics, Inc.) to screen for expression of ApoER2 and Reelin RNAs. All sections to be sequentially processed for iterative MP-IHC screening were first incubated with Human BD Fc Blocking solution (BD Biosciences) to saturate endogenous Fc receptors, and then in True Black Reagent (Biotium) to quench intrinsic tissue autofluorescence. The sections were then immunoreacted for 1 hour at RT using $1-5 \mu \mathrm{g} / \mathrm{ml}$ cocktail mixture of immune-compatible antibody panels targeting ApoE/Reelin-ApoE receptor-DAB1 signaling cascade pathways, classical biomarkers of AD pathology, and brain cytoarchitectural biomarkers (see Table S1 for antibody sources and technical specifications). This step was followed by washing off excess primary antibodies in PBS supplemented with $1 \mathrm{mg} / \mathrm{ml}$ bovine serum albumin (BSA) and staining the sections using a 1 $\mu \mathrm{g} / \mathrm{ml}$ cocktail mixture of the appropriately cross-adsorbed secondary antibodies (purchased from either Thermo Fisher, Jackson ImmunoResearch or Li-Cor Biosciences) conjugated to one of the following spectrally compatible fluorophores: Alexa Fluor 430, Alexa Fluor 488, Alexa Fluor 546, Alexa Fluor 594, Alexa Fluor 
medRxiv preprint doi: https://doi.org/10.1101/2021.07.05.21259649; this version posted July 6, 2021. The copyright holder for this preprint

(which was not certified by peer review) is the author/funder, who has granted medRxiv a license to display the preprint in perpetuity.

This article is a US Government work. It is not subject to copyright under 17 USC 105 and is also made available for use under a CCO license.

647, IRDye 600LT, or IRDye 800CW. After washing off excess secondary antibodies, sections were

counterstained using $1 \mu \mathrm{g} / \mathrm{ml}$ DAPI (Thermo Fisher Scientific) for visualization of cell nuclei. Slides were then coverslipped using Immu-Mount medium (Thermo Fisher Scientific) and imaged using a multispectral epifluorescence microscope (see below). After imaging, tissue bound primary and secondary antibodies were both stripped off the slides after a 5-minute incubation at RT in NewBlot Nitro 5X Stripping buffer (Li-Cor Biosciences) followed by 1-minute additional heat mediated antigen retrieval step in Tris/EDTA buffer. The above processing cycle beginning with re-blocking of tissues in Human BD Fc Blocking solution was repeated and the same sections then incubated using an additional panel of antibodies of interest.

\section{Multiplex Fluorescence Immunohistochemistry Image Acquisition and Computational Reconstruction}

Images were acquired from MP-IHC and/or MP-ISH probed whole specimen sections using the Axio Imager.Z2 slide scanning fluorescence microscope (Zeiss) equipped with a 20X/0.8 Plan-Apochromat (Phase-2) nonimmersion objective (Zeiss), a high resolution ORCA-Flash4.0 sCMOS digital camera (Hamamatsu), a 200W X-Cite 200DC broad band lamp source (Excelitas Technologies), and 8 customized filter sets (Semrock) optimized to detect the following fluorophores: DAPI, Alexa Fluor 430, Alexa Fluor 488 (or Opal 520), Alexa Fluor 546 (or Opal 570), Alexa Fluor 594 (or Opal 620), Alexa Fluor 647, IRDye 680LT (or Opal 690) and IRDye $800 \mathrm{CW}$. Image tiles $(600 \times 600 \mu \mathrm{m}$ viewing area) were individually captured at $0.325 \mathrm{micron} / \mathrm{pixel}$ spatial resolution, and the tiles seamlessly stitched into whole specimen images using the ZEN 2 image acquisition and analysis software program (Zeiss), with an appropriate color table having been applied to each image channel to either match its emission spectrum or to set a distinguishing color balance. Pseudocolored stitched images acquired from 1 round of MP-ISH and/or up to 6 rounds of MP-IHC staining and imaging were then exported to Adobe Photoshop and overlaid as individual layers to create multicolored merged composites. Multi-channel images acquired from multi-round IHC staining and repeatedly imaged tissue samples were computationally registered at the subpixel level using affine transformation, and the aligned fluorescence images corrected for photobleaching, autofluorescence, background signals, non-uniform illumination, and spectral bleed-through artifacts, as previously described by Maric et al. ${ }^{176}$

\section{Confocal Microscopy}

A Zeiss LSM 710 confocal microscope equipped with an Apochromat 100x/NA 1.4 oil objective was used to image sections. Brightness and Contrast were adjusted with the Auto B/C function of FIJI/Image J software (https://imagej.nih.gov/ij/index.htm).

\section{Regional Annotations and Area Quantitation}


medRxiv preprint doi: https://doi.org/10.1101/2021.07.05.21259649; this version posted July 6, 2021. The copyright holder for this preprint (which was not certified by peer review) is the author/funder, who has granted medRxiv a license to display the preprint in perpetuity.

This article is a US Government work. It is not subject to copyright under 17 USC 105 and is also made available for use under a CCO license.

Corrected MP-IHC images and traditional single-marker IHC images of coronal sections of the medial temporal lobe at the body of the hippocampus were uploaded into HALO 3.1 image analysis software (Indica Labs, Corrales, NM). Boundaries of each anatomical region of interest (entorhinal cortex, hippocampus, and dentate gyrus including the molecular layer) were identified and annotated using a combination of anatomical landmarks (the lateral ventricle, fimbria, fimbria-dentate and hippocampal fissures, collateral sulcus, dentate gyrus, cornu ammonis) and cytoarchitectonic mapping as described by Amunts et al. ${ }^{177}$ MP-IHC, which enabled immunolabeling of numerous cytoarchitectural markers in each section (Table S1), assisted in the identification of anatomical landmarks such as the dentate gyrus, and boundaries including the gray matter-white matter interface. HALO Area Quantification v2.1.5 and Area Quantification Fluorescence v2.1.7 modules were applied to MP-IHC and single-marker IHC-generated images, respectively, to quantify stain positive area as a percentage of each annotated region.

\section{Statistical analysis}

For each annotated region, the Spearman's correlation coefficient between each immunohistochemical marker (area percentage positive stain) and each Alzheimer's disease endpoint (MMSE, Braak score, Total Tangle, Total A $\beta$ plaque load, Neuritic plaque density) was calculated using Stata Release 16 (College Station, TX). No adjustments were made for multiple comparisons. 
medRxiv preprint doi: https://doi.org/10.1101/2021.07.05.21259649; this version posted July 6, 2021. The copyright holder for this preprint

(which was not certified by peer review) is the author/funder, who has granted medRxiv a license to display the preprint in perpetuity.

This article is a US Government work. It is not subject to copyright under 17 USC 105 and is also made available

\section{Declaration of competing interest} for use under a CCO license.

The authors report no declarations of interest.

\section{Acknowledgements}

This work was supported by the intramural programs of the National Institute on Aging (NIA), National Institute on Alcohol Abuse and Alcoholism (NIAAA) and National Institute on Neurological Diseases and Stroke (NINDS), NIH. Additional support was provided as a research gift from John M. Davis to the Laboratory of Clinical Investigation, NIA/NIH. We are grateful to the Banner Sun Health Research Institute Brain and Body Donation Program of Sun City, Arizona for the provision of human brain tissue. The Brain and Body Donation Program is supported by the NINDS (U24 NS072026 National Brain and Tissue Resource for Parkinson's Disease and Related Disorders), the NIA (P30 AG19610 Arizona Alzheimer's Disease Core Center), the Arizona Department of Health Services (contract 211002, Arizona Alzheimer's Research Center), the Arizona Biomedical Research Commission (contracts 4001, 0011, 05-901 and 1001 to the Arizona Parkinson's Disease Consortium) and the Michael J. Fox Foundation for Parkinson's Research. We gratefully acknowledge the volunteer participants and their families who provided postmortem tissues, as well as Geidy Serrano, Thomas Beach and the BBDP on-call autopsy team who provided this extraordinary resource. Special thanks to Pragun Vohra and the Histoserv staff for immunohistochemical expertise and immunostaining, to Scott Lewis and New England Peptide/Vivitide staff for expert input into antibody development, purification, and validation, and to Precision Antibody for expert input into monoclonal antibody development. We are grateful to Josephine Egan (Chief, Laboratory of Clinical Investigation, NIA) for reading and editing the manuscript. The authors acknowledge the valuable published work of investigators that provided a foundation for the present hypotheses. We also thank the National Library of Medicine and PubMed for enabling userfriendly access to published literature and the NIH Interlibrary Loan staff for obtaining literature not indexed in PubMed. 
medRxiv preprint doi: https://doi.org/10.1101/2021.07.05.21259649; this version posted July 6, 2021. The copyright holder for this preprint

(which was not certified by peer review) is the author/funder, who has granted medRxiv a license to display the preprint in perpetuity. This article is a US Government work. It is not subject to copyright under 17 USC 105 and is also made available for use under a CCO license.

\section{REFERENCES}

1. Querfurth HW, LaFerla FM. Alzheimer's disease. N Engl J Med 2010;362(4):329-44. doi: 10.1056/NEJMra0909142 [published Online First: 2010/01/29]

2. McKhann GM, Knopman DS, Chertkow $\mathrm{H}$, et al. The diagnosis of dementia due to Alzheimer's disease: recommendations from the National Institute on Aging-Alzheimer's Association workgroups on diagnostic guidelines for Alzheimer's disease. Alzheimers Dement 2011;7(3):263-9. doi: 10.1016/j.jalz.2011.03.005 [published Online First: 2011/04/26]

3. Scheff SW, Sparks DL, Price DA. Quantitative assessment of synaptic density in the outer molecular layer of the hippocampal dentate gyrus in Alzheimer's disease. Dementia 1996;7(4):226-32. doi: 10.1159/000106884 [published Online First: 1996/07/01]

4. Scheff SW, Price DA. Synaptic density in the inner molecular layer of the hippocampal dentate gyrus in Alzheimer disease. J Neuropathol Exp Neurol 1998;57(12):1146-53. doi: 10.1097/00005072-199812000-00006 [published Online First: 1998/12/23]

5. Coleman PD, Yao PJ. Synaptic slaughter in Alzheimer's disease. Neurobiol Aging 2003;24(8):1023-7. doi: 10.1016/j.neurobiolaging.2003.09.001 [published Online First: 2003/12/04]

6. Kobro-Flatmoen A, Nagelhus A, Witter MP. Reelin-immunoreactive neurons in entorhinal cortex layer II selectively express intracellular amyloid in early Alzheimer's disease. Neurobiol Dis 2016;93:172-83. doi: 10.1016/j.nbd.2016.05.012 [published Online First: 2016/05/20]

7. Kordower JH, Chu Y, Stebbins GT, et al. Loss and atrophy of layer II entorhinal cortex neurons in elderly people with mild cognitive impairment. Ann Neurol 2001;49(2):202-13. [published Online First: 2001/02/28]

8. Van Hoesen GW, Hyman BT, Damasio AR. Entorhinal cortex pathology in Alzheimer's disease. Hippocampus 1991;1(1):1-8. doi: 10.1002/hipo.450010102 [published Online First: 1991/01/01]

9. Corder EH, Saunders AM, Strittmatter WJ, et al. Gene dose of apolipoprotein E type 4 allele and the risk of Alzheimer's disease in late onset families. Science 1993;261(5123):921-3. doi: 10.1126/science.8346443 [published Online First: 1993/08/13]

10. Strittmatter WJ, Saunders AM, Schmechel D, et al. Apolipoprotein E: high-avidity binding to beta-amyloid and increased frequency of type 4 allele in late-onset familial Alzheimer disease. Proc Natl Acad Sci U S A 1993;90(5):1977-81. doi: 10.1073/pnas.90.5.1977 [published Online First: 1993/03/01]

11. Rebeck GW, Reiter JS, Strickland DK, et al. Apolipoprotein E in sporadic Alzheimer's disease: allelic variation and receptor interactions. Neuron 1993;11(4):575-80. doi: 10.1016/0896-6273(93)90070-8 [published Online First: 1993/10/01]

12. Kunkle BW, Grenier-Boley B, Sims R, et al. Genetic meta-analysis of diagnosed Alzheimer's disease identifies new risk loci and implicates Abeta, tau, immunity and lipid processing. Nat Genet 2019;51(3):414-30. doi: 10.1038/s41588-019-0358-2 [published Online First: 2019/03/02]

13. Mauch DH, Nagler K, Schumacher S, et al. CNS synaptogenesis promoted by glia-derived cholesterol. Science 2001;294(5545):1354-7. doi: 10.1126/science.294.5545.1354 [published Online First: 2001/11/10]

14. Zhu YB, Gao W, Zhang Y, et al. Astrocyte-derived phosphatidic acid promotes dendritic branching. Sci Rep 2016;6:21096. doi: 10.1038/srep21096 [published Online First: 2016/02/18]

15. Igbavboa U, Hamilton J, Kim HY, et al. A new role for apolipoprotein E: modulating transport of polyunsaturated phospholipid molecular species in synaptic plasma membranes. J Neurochem 2002;80(2):255-61. doi: 10.1046/j.0022-3042.2001.00688.x [published Online First: 2002/03/21]

16. Kruger MT, Zhao S, Chai X, et al. Role for Reelin-induced cofilin phosphorylation in the assembly of sympathetic preganglionic neurons in the murine intermediolateral column. Eur J Neurosci 2010;32(10):1611-7. doi: 10.1111/j.1460-9568.2010.07433.x [published Online First: 2010/11/03]

17. Hiesberger T, Trommsdorff M, Howell BW, et al. Direct binding of Reelin to VLDL receptor and ApoE receptor 2 induces tyrosine phosphorylation of disabled-1 and modulates tau phosphorylation. Neuron 1999;24(2):481-9. doi: 10.1016/s0896-6273(00)80861-2 [published Online First: 1999/11/26]

18. Trotter J, Lee GH, Kazdoba TM, et al. Dab1 is required for synaptic plasticity and associative learning. J Neurosci 2013;33(39):15652-68. doi: 10.1523/JNEUROSCI.2010-13.2013 [published Online First: 2013/09/27]

19. Bosch C, Muhaisen A, Pujadas L, et al. Reelin Exerts Structural, Biochemical and Transcriptional Regulation Over Presynaptic and Postsynaptic Elements in the Adult Hippocampus. Front Cell Neurosci 2016;10:138. doi: 10.3389/fncel.2016.00138 [published Online First: 2016/06/16] 
medRxiv preprint doi: https://doi.org/10.1101/2021.07.05.21259649; this version posted July 6 , 2021. The copyright holder for this preprint

(which was not certified by peer review) is the author/funder, who has granted medRxiv a license to display the preprint in perpetuity. This article is a US Government work. It is not subject to copyright under 17 USC 105 and is also made available for use under a CCO license.

20. Ventruti A, Kazdoba TM, Niu S, et al. Reelin deficiency causes specific defects in the molecular composition of the synapses in the adult brain. Neuroscience 2011;189:32-42. doi: 10.1016/j.neuroscience.2011.05.050 [published Online First: 2011/06/15]

21. Wasser CR, Herz J. Reelin: Neurodevelopmental Architect and Homeostatic Regulator of Excitatory Synapses. J Biol Chem 2017;292(4):1330-38. doi: 10.1074/jbc.R116.766782 [published Online First: 2016/12/21]

22. Kim DH, lijima H, Goto K, et al. Human apolipoprotein E receptor 2. A novel lipoprotein receptor of the low density lipoprotein receptor family predominantly expressed in brain. J Biol Chem 1996;271(14):8373-80. doi: 10.1074/jbc.271.14.8373 [published Online First: 1996/04/05]

23. Duit S, Mayer H, Blake SM, et al. Differential functions of ApoER2 and very low density lipoprotein receptor in Reelin signaling depend on differential sorting of the receptors. J Biol Chem 2010;285(7):4896-908. doi: 10.1074/jbc.M109.025973 [published Online First: 2009/12/02]

24. Riddell DR, Sun XM, Stannard AK, et al. Localization of apolipoprotein E receptor 2 to caveolae in the plasma membrane. J Lipid Res 2001;42(6):998-1002. [published Online First: 2001/05/23]

25. Dumanis SB, Cha HJ, Song JM, et al. ApoE receptor 2 regulates synapse and dendritic spine formation. PLoS One 2011;6(2):e17203. doi: 10.1371/journal.pone.0017203 [published Online First: 2011/02/25]

26. Beffert U, Weeber EJ, Durudas A, et al. Modulation of synaptic plasticity and memory by Reelin involves differential splicing of the lipoprotein receptor Apoer2. Neuron 2005;47(4):567-79. doi: 10.1016/j.neuron.2005.07.007 [published Online First: 2005/08/17]

27. Petit-Turcotte $\mathrm{C}$, Aumont $\mathrm{N}$, Beffert $\mathrm{U}$, et al. The apoE receptor apoER2 is involved in the maintenance of efficient synaptic plasticity. Neurobiol Aging 2005;26(2):195-206. doi: 10.1016/j.neurobiolaging.2004.04.007 [published Online First: 2004/12/08]

28. Lane-Donovan C, Philips GT, Herz J. More than cholesterol transporters: lipoprotein receptors in CNS function and neurodegeneration. Neuron 2014;83(4):771-87. doi: 10.1016/j.neuron.2014.08.005 [published Online First: 2014/08/22]

29. Qiu S, Korwek KM, Weeber EJ. A fresh look at an ancient receptor family: emerging roles for low density lipoprotein receptors in synaptic plasticity and memory formation. Neurobiol Learn Mem 2006;85(1):16-29. doi: 10.1016/j.nlm.2005.08.009 [published Online First: 2005/10/04]

30. Koudinov AR, Koudinova NV. Essential role for cholesterol in synaptic plasticity and neuronal degeneration. FASEB J 2001;15(10):1858-60. doi: 10.1096/fj.00-0815fje [published Online First: 2001/08/02]

31. Markesbery WR, Kryscio RJ, Lovell MA, et al. Lipid peroxidation is an early event in the brain in amnestic mild cognitive impairment. Ann Neurol 2005;58(5):730-5. doi: 10.1002/ana.20629 [published Online First: 2005/10/22]

32. Smith MA, Perry G, Richey PL, et al. Oxidative damage in Alzheimer's. Nature 1996;382(6587):120-1. doi: 10.1038/382120b0 [published Online First: 1996/07/11]

33. Sayre LM, Zelasko DA, Harris PL, et al. 4-Hydroxynonenal-derived advanced lipid peroxidation end products are increased in Alzheimer's disease. J Neurochem 1997;68(5):2092-7. doi: 10.1046/j.1471-4159.1997.68052092.x [published Online First: 1997/05/01]

34. Fukuda M, Kanou F, Shimada N, et al. Elevated levels of 4-hydroxynonenal-histidine Michael adduct in the hippocampi of patients with Alzheimer's disease. Biomed Res 2009;30(4):227-33. doi:

10.2220/biomedres.30.227 [published Online First: 2009/09/05]

35. Markesbery WR. Oxidative stress hypothesis in Alzheimer's disease. Free Radic Biol Med 1997;23(1):134-47. doi: 10.1016/s0891-5849(96)00629-6 [published Online First: 1997/01/01]

36. Montine KS, Olson SJ, Amarnath V, et al. Immunohistochemical detection of 4-hydroxy-2-nonenal adducts in Alzheimer's disease is associated with inheritance of APOE4. Am J Pathol 1997;150(2):437-43. [published Online First: 1997/02/01]

37. Calingasan NY, Uchida K, Gibson GE. Protein-bound acrolein: a novel marker of oxidative stress in Alzheimer's disease. J Neurochem 1999;72(2):751-6. doi: 10.1046/j.1471-4159.1999.0720751.x [published Online First: 1999/02/04]

38. Liu Q, Raina AK, Smith MA, et al. Hydroxynonenal, toxic carbonyls, and Alzheimer disease. Mol Aspects Med 2003;24(4-5):305-13. doi: 10.1016/s0098-2997(03)00025-6 [published Online First: 2003/08/02]

39. Uchida K. Histidine and lysine as targets of oxidative modification. Amino Acids 2003;25(3-4):249-57. doi: 10.1007/s00726-003-0015-y [published Online First: 2003/12/09] 
medRxiv preprint doi: https://doi.org/10.1101/2021.07.05.21259649; this version posted July 6, 2021. The copyright holder for this preprint

(which was not certified by peer review) is the author/funder, who has granted medRxiv a license to display the preprint in perpetuity. This article is a US Government work. It is not subject to copyright under 17 USC 105 and is also made available for use under a CCO license.

40. Maeshima T, Honda K, Chikazawa M, et al. Quantitative analysis of acrolein-specific adducts generated during lipid peroxidation-modification of proteins in vitro: identification of $\mathrm{N}(\mathrm{tau})$-(3-propanal)histidine as the major adduct. Chem Res Toxicol 2012;25(7):1384-92. doi: 10.1021/tx3000818 [published Online First: 2012/06/22]

41. Yasui N, Nogi T, Takagi J. Structural basis for specific recognition of reelin by its receptors. Structure 2010;18(3):32031. doi: 10.1016/j.str.2010.01.010 [published Online First: 2010/03/13]

42. Fisher C, Beglova N, Blacklow SC. Structure of an LDLR-RAP complex reveals a general mode for ligand recognition by lipoprotein receptors. Mol Cell 2006;22(2):277-83. doi: 10.1016/j.molcel.2006.02.021 [published Online First: 2006/04/25]

43. Hirai H, Yasui N, Yamashita K, et al. Structural basis for ligand capture and release by the endocytic receptor ApoER2. EMBO Rep 2017;18(6):982-99. doi: 10.15252/embr.201643521 [published Online First: 2017/04/28]

44. Yasui N, Nogi T, Kitao T, et al. Structure of a receptor-binding fragment of reelin and mutational analysis reveal a recognition mechanism similar to endocytic receptors. Proc Natl Acad Sci U S A 2007;104(24):9988-93. doi: 10.1073/pnas.0700438104 [published Online First: 2007/06/06]

45. Andersen OM, Benhayon D, Curran T, et al. Differential binding of ligands to the apolipoprotein E receptor 2. Biochemistry 2003;42(31):9355-64. doi: 10.1021/bi034475p [published Online First: 2003/08/06]

46. D'Arcangelo G, Homayouni R, Keshvara L, et al. Reelin is a ligand for lipoprotein receptors. Neuron 1999;24(2):471-9. doi: 10.1016/s0896-6273(00)80860-0 [published Online First: 1999/11/26]

47. Nelson CD, Kim MJ, Hsin H, et al. Phosphorylation of threonine-19 of PSD-95 by GSK-3beta is required for PSD-95 mobilization and long-term depression. J Neurosci 2013;33(29):12122-35. doi: 10.1523/JNEUROSCI.013113.2013 [published Online First: 2013/07/19]

48. May-Zhang LS, Yermalitsky V, Melchior JT, et al. Modified sites and functional consequences of 4-oxo-2-nonenal adducts in HDL that are elevated in familial hypercholesterolemia. J Biol Chem 2019;294(50):19022-33. doi: 10.1074/jbc.RA119.009424 [published Online First: 2019/11/02]

49. Sayre LM, Lin D, Yuan Q, et al. Protein adducts generated from products of lipid oxidation: focus on HNE and one. Drug Metab Rev 2006;38(4):651-75. doi: 10.1080/03602530600959508 [published Online First: 2006/12/06]

50. Xu G, Liu Y, Kansal MM, et al. Rapid cross-linking of proteins by 4-ketoaldehydes and 4-hydroxy-2-alkenals does not arise from the lysine-derived monoalkylpyrroles. Chem Res Toxicol 1999;12(9):855-61. doi: 10.1021/tx990056a [published Online First: 1999/09/22]

51. Liu Z, Minkler PE, Sayre LM. Mass spectroscopic characterization of protein modification by 4-hydroxy-2-(E)-nonenal and 4-oxo-2-(E)-nonenal. Chem Res Toxicol 2003;16(7):901-11. doi: 10.1021/tx0300030 [published Online First: 2003/07/23]

52. Fernandez RF, Ellis JM. Acyl-CoA synthetases as regulators of brain phospholipid acyl-chain diversity. Prostaglandins Leukot Essent Fatty Acids 2020;161:102175. doi: 10.1016/j.plefa.2020.102175 [published Online First: 2020/10/09]

53. Mirra SS, Heyman A, McKeel D, et al. The Consortium to Establish a Registry for Alzheimer's Disease (CERAD). Part II. Standardization of the neuropathologic assessment of Alzheimer's disease. Neurology 1991;41(4):479-86. doi: 10.1212/wnl.41.4.479 [published Online First: 1991/04/01]

54. Consensus recommendations for the postmortem diagnosis of Alzheimer's disease. The National Institute on Aging, and Reagan Institute Working Group on Diagnostic Criteria for the Neuropathological Assessment of Alzheimer's Disease. Neurobiol Aging 1997;18(4 Suppl):S1-2. [published Online First: 1997/07/01]

55. Hoe HS, Pocivavsek A, Chakraborty G, et al. Apolipoprotein E receptor 2 interactions with the N-methyl-D-aspartate receptor. J Biol Chem 2006;281(6):3425-31. doi: 10.1074/jbc.M509380200 [published Online First: 2005/12/08]

56. Gallo CM, Ho A, Beffert U. ApoER2: Functional Tuning Through Splicing. Front Mol Neurosci 2020;13:144. doi: 10.3389/fnmol.2020.00144 [published Online First: 2020/08/28]

57. Hyman BT, Van Hoesen GW, Kromer LJ, et al. Perforant pathway changes and the memory impairment of Alzheimer's disease. Ann Neurol 1986;20(4):472-81. doi: 10.1002/ana.410200406 [published Online First: 1986/10/01]

58. Morys J, Sadowski M, Barcikowska M, et al. The second layer neurones of the entorhinal cortex and the perforant path in physiological ageing and Alzheimer's disease. Acta Neurobiol Exp (Wars) 1994;54(1):47-53. [published Online First: 1994/01/01]

59. Woods NI, Vaaga CE, Chatzi C, et al. Preferential Targeting of Lateral Entorhinal Inputs onto Newly Integrated Granule Cells. J Neurosci 2018;38(26):5843-53. doi: 10.1523/JNEUROSCI.1737-17.2018 [published Online First: 2018/05/26] 
medRxiv preprint doi: https://doi.org/10.1101/2021.07.05.21259649; this version posted July 6 , 2021. The copyright holder for this preprint

(which was not certified by peer review) is the author/funder, who has granted medRxiv a license to display the preprint in perpetuity. This article is a US Government work. It is not subject to copyright under 17 USC 105 and is also made available for use under a CCO license.

60. Bock HH, Jossin Y, May P, et al. Apolipoprotein E receptors are required for reelin-induced proteasomal degradation of the neuronal adaptor protein Disabled-1. J Biol Chem 2004;279(32):33471-9. doi: 10.1074/jbc.M401770200 [published Online First: 2004/06/04]

61. Dickson TC, Saunders HL, Vickers JC. Relationship between apolipoprotein E and the amyloid deposits and dystrophic neurites of Alzheimer's disease. Neuropathol Appl Neurobiol 1997;23(6):483-91. doi: 10.1111/j.13652990.1997.tb01325.x [published Online First: 1998/02/14]

62. Folstein MF, Folstein SE, McHugh PR. "Mini-mental state". A practical method for grading the cognitive state of patients for the clinician. J Psychiatr Res 1975;12(3):189-98. doi: 10.1016/0022-3956(75)90026-6 [published Online First: 1975/11/01]

63. Braak H, Braak E. Neuropathological stageing of Alzheimer-related changes. Acta Neuropathol 1991;82(4):239-59. doi: 10.1007/BF00308809 [published Online First: 1991/01/01]

64. Braak H, Alafuzoff I, Arzberger T, et al. Staging of Alzheimer disease-associated neurofibrillary pathology using paraffin sections and immunocytochemistry. Acta Neuropathol 2006;112(4):389-404. doi: 10.1007/s00401-0060127-z [published Online First: 2006/08/15]

65. Beach TG, Adler CH, Sue LI, et al. Arizona Study of Aging and Neurodegenerative Disorders and Brain and Body Donation Program. Neuropathology 2015;35(4):354-89. doi: 10.1111/neup.12189 [published Online First: 2015/01/27]

66. Beffert U, Morfini G, Bock HH, et al. Reelin-mediated signaling locally regulates protein kinase B/Akt and glycogen synthase kinase 3beta. J Biol Chem 2002;277(51):49958-64. doi: 10.1074/jbc.M209205200 [published Online First: 2002/10/12]

67. Ohkubo N, Lee YD, Morishima A, et al. Apolipoprotein E and Reelin ligands modulate tau phosphorylation through an apolipoprotein E receptor/disabled-1/glycogen synthase kinase-3beta cascade. FASEB J 2003;17(2):295-7. doi: 10.1096/fj.02-0434fje [published Online First: 2002/12/20]

68. Kocherhans S, Madhusudan A, Doehner J, et al. Reduced Reelin expression accelerates amyloid-beta plaque formation and tau pathology in transgenic Alzheimer's disease mice. J Neurosci 2010;30(27):9228-40. doi: 10.1523/JNEUROSCI.0418-10.2010 [published Online First: 2010/07/09]

69. Pujadas $L$, Rossi $D$, Andres $R$, et al. Reelin delays amyloid-beta fibril formation and rescues cognitive deficits in a model of Alzheimer's disease. Nat Commun 2014;5:3443. doi: 10.1038/ncomms4443 [published Online First: 2014/03/07]

70. Deutsch SI, Rosse RB, Deutsch LH. Faulty regulation of tau phosphorylation by the reelin signal transduction pathway is a potential mechanism of pathogenesis and therapeutic target in Alzheimer's disease. Eur Neuropsychopharmacol 2006;16(8):547-51. doi: 10.1016/j.euroneuro.2006.01.006 [published Online First: 2006/03/01]

71. Cuchillo-Ibanez I, Balmaceda V, Botella-Lopez A, et al. Beta-amyloid impairs reelin signaling. PLoS One 2013;8(8):e72297. doi: 10.1371/journal.pone.0072297 [published Online First: 2013/08/21]

72. Cuchillo-lbanez I, Mata-Balaguer T, Balmaceda V, et al. The beta-amyloid peptide compromises Reelin signaling in Alzheimer's disease. Sci Rep 2016;6:31646. doi: 10.1038/srep31646 [published Online First: 2016/08/18]

73. Chai $X$, Forster $E$, Zhao $S$, et al. Reelin stabilizes the actin cytoskeleton of neuronal processes by inducing $n$-cofilin phosphorylation at serine3. J Neurosci 2009;29(1):288-99. doi: 10.1523/JNEUROSCI.2934-08.2009 [published Online First: 2009/01/09]

74. Frotscher M, Zhao S, Wang S, et al. Reelin Signaling Inactivates Cofilin to Stabilize the Cytoskeleton of Migrating Cortical Neurons. Front Cell Neurosci 2017;11:148. doi: 10.3389/fncel.2017.00148 [published Online First: 2017/06/08]

75. Niu S, Renfro A, Quattrocchi CC, et al. Reelin promotes hippocampal dendrite development through the VLDLR/ApoER2-Dab1 pathway. Neuron 2004;41(1):71-84. doi: 10.1016/s0896-6273(03)00819-5 [published Online First: 2004/01/13]

76. Nixon RA. Amyloid precursor protein and endosomal-lysosomal dysfunction in Alzheimer's disease: inseparable partners in a multifactorial disease. FASEB J 2017;31(7):2729-43. doi: 10.1096/fj.201700359 [published Online First: 2017/07/01]

77. Kawai M, Cras P, Richey P, et al. Subcellular localization of amyloid precursor protein in senile plaques of Alzheimer's disease. Am J Pathol 1992;140(4):947-58. [published Online First: 1992/04/01]

78. Malik BR, Maddison DC, Smith GA, et al. Autophagic and endo-lysosomal dysfunction in neurodegenerative disease. Mol Brain 2019;12(1):100. doi: 10.1186/s13041-019-0504-x [published Online First: 2019/12/01] 
medRxiv preprint doi: https://doi.org/10.1101/2021.07.05.21259649; this version posted July 6, 2021. The copyright holder for this preprint

(which was not certified by peer review) is the author/funder, who has granted medRxiv a license to display the preprint in perpetuity. This article is a US Government work. It is not subject to copyright under 17 USC 105 and is also made available for use under a CCO license.

79. Kandalepas PC, Sadleir KR, Eimer WA, et al. The Alzheimer's beta-secretase BACE1 localizes to normal presynaptic terminals and to dystrophic presynaptic terminals surrounding amyloid plaques. Acta Neuropathol 2013;126(3):329-52. doi: 10.1007/s00401-013-1152-3 [published Online First: 2013/07/04]

80. Gowrishankar S, Yuan $\mathrm{P}, \mathrm{Wu} \mathrm{Y}$, et al. Massive accumulation of luminal protease-deficient axonal lysosomes at Alzheimer's disease amyloid plaques. Proc Natl Acad Sci U S A 2015;112(28):E3699-708. doi: 10.1073/pnas.1510329112 [published Online First: 2015/07/01]

81. Su JH, Cummings BJ, Cotman CW. Identification and distribution of axonal dystrophic neurites in Alzheimer's disease. Brain Res 1993;625(2):228-37. doi: 10.1016/0006-8993(93)91063-x [published Online First: 1993/10/22]

82. Takahashi RH, Capetillo-Zarate E, Lin MT, et al. Accumulation of intraneuronal beta-amyloid 42 peptides is associated with early changes in microtubule-associated protein 2 in neurites and synapses. PLoS One 2013;8(1):e51965. doi: 10.1371/journal.pone.0051965 [published Online First: 2013/02/02]

83. Reiman EM, Arboleda-Velasquez JF, Quiroz YT, et al. Exceptionally low likelihood of Alzheimer's dementia in APOE2 homozygotes from a 5,000-person neuropathological study. Nat Commun 2020;11(1):667. doi: 10.1038/s41467019-14279-8 [published Online First: 2020/02/06]

84. Namba Y, Tomonaga M, Kawasaki H, et al. Apolipoprotein E immunoreactivity in cerebral amyloid deposits and neurofibrillary tangles in Alzheimer's disease and kuru plaque amyloid in Creutzfeldt-Jakob disease. Brain Res 1991;541(1):163-6. doi: 10.1016/0006-8993(91)91092-f [published Online First: 1991/02/08]

85. Thal DR, Capetillo-Zarate E, Schultz C, et al. Apolipoprotein E co-localizes with newly formed amyloid beta-protein (Abeta) deposits lacking immunoreactivity against $\mathrm{N}$-terminal epitopes of Abeta in a genotype-dependent manner. Acta Neuropathol 2005;110(5):459-71. doi: 10.1007/s00401-005-1053-1 [published Online First: 2005/10/01]

86. Sheng JG, Mrak RE, Griffin WS. Apolipoprotein E distribution among different plaque types in Alzheimer's disease: implications for its role in plaque progression. Neuropathol Appl Neurobiol 1996;22(4):334-41. doi: 10.1111/j.1365-2990.1996.tb01112.x [published Online First: 1996/08/01]

87. Malek-Ahmadi M, Perez SE, Chen K, et al. Neuritic and Diffuse Plaque Associations with Memory in Non-Cognitively Impaired Elderly. J Alzheimers Dis 2016;53(4):1641-52. doi: 10.3233/JAD-160365 [published Online First: 2016/08/20]

88. Nelson PT, Alafuzoff I, Bigio EH, et al. Correlation of Alzheimer disease neuropathologic changes with cognitive status: a review of the literature. J Neuropathol Exp Neurol 2012;71(5):362-81. doi: 10.1097/NEN.0b013e31825018f7 [published Online First: 2012/04/11]

89. Pfennig S, Foss F, Bissen D, et al. GRIP1 Binds to ApoER2 and EphrinB2 to Induce Activity-Dependent AMPA Receptor Insertion at the Synapse. Cell Rep 2017;21(1):84-96. doi: 10.1016/j.celrep.2017.09.019 [published Online First: 2017/10/06]

90. Cuitino L, Matute R, Retamal C, et al. ApoER2 is endocytosed by a clathrin-mediated process involving the adaptor protein Dab2 independent of its Rafts' association. Traffic 2005;6(9):820-38. doi: 10.1111/j.16000854.2005.00320.x [published Online First: 2005/08/17]

91. Wasser CR, Masiulis I, Durakoglugil MS, et al. Differential splicing and glycosylation of Apoer2 alters synaptic plasticity and fear learning. Sci Signal 2014;7(353):ra113. doi: 10.1126/scisignal.2005438 [published Online First: 2014/11/28]

92. Weeber EJ, Beffert U, Jones $C$, et al. Reelin and ApoE receptors cooperate to enhance hippocampal synaptic plasticity and learning. J Biol Chem 2002;277(42):39944-52. doi: 10.1074/jbc.M205147200 [published Online First: 2002/08/09]

93. Qiu S, Weeber EJ. Reelin signaling facilitates maturation of CA1 glutamatergic synapses. J Neurophysiol 2007;97(3):2312-21. doi: 10.1152/jn.00869.2006 [published Online First: 2007/01/19]

94. Hinrich AJ, Jodelka FM, Chang JL, et al. Therapeutic correction of ApoER2 splicing in Alzheimer's disease mice using antisense oligonucleotides. EMBO Mol Med 2016;8(4):328-45. doi: 10.15252/emmm.201505846 [published Online First: 2016/02/24]

95. Motoi Y, Itaya M, Mori H, et al. Apolipoprotein E receptor 2 is involved in neuritic plaque formation in APP sw mice. Neurosci Lett 2004;368(2):144-7. doi: 10.1016/j.neulet.2004.06.081 [published Online First: 2004/09/08]

96. Motoi $\mathrm{Y}$, Iwasaki T, Hattori $\mathrm{H}$, et al. Neuronal apolipoprotein e receptor 2 immunoreactivity in Alzheimer's disease. Alzheimer's Reports 1999;2(2):87-91. 
medRxiv preprint doi: https://doi.org/10.1101/2021.07.05.21259649; this version posted July 6,2021 . The copyright holder for this preprint

(which was not certified by peer review) is the author/funder, who has granted medRxiv a license to display the preprint in perpetuity. This article is a US Government work. It is not subject to copyright under 17 USC 105 and is also made available for use under a CCO license.

97. Llorens-Martin M, Blazquez-Llorca L, Benavides-Piccione $\mathrm{R}$, et al. Selective alterations of neurons and circuits related to early memory loss in Alzheimer's disease. Front Neuroanat 2014;8:38. doi: 10.3389/fnana.2014.00038 [published Online First: 2014/06/07]

98. Thal DR, Holzer M, Rub U, et al. Alzheimer-related tau-pathology in the perforant path target zone and in the hippocampal stratum oriens and radiatum correlates with onset and degree of dementia. Exp Neurol 2000;163(1):98-110. doi: 10.1006/exnr.2000.7380 [published Online First: 2000/04/29]

99. Martinez-Cerdeno V, Galazo MJ, Clasca F. Reelin-immunoreactive neurons, axons, and neuropil in the adult ferret brain: evidence for axonal secretion of reelin in long axonal pathways. J Comp Neurol 2003;463(1):92-116. doi: 10.1002/cne.10748 [published Online First: 2003/06/18]

100. Ramos-Moreno T, Galazo MJ, Porrero C, et al. Extracellular matrix molecules and synaptic plasticity: immunomapping of intracellular and secreted Reelin in the adult rat brain. Eur J Neurosci 2006;23(2):401-22. doi: 10.1111/j.1460-9568.2005.04567.x [published Online First: 2006/01/20]

101. Martinez-Cerdeno V, Galazo MJ, Cavada C, et al. Reelin immunoreactivity in the adult primate brain: intracellular localization in projecting and local circuit neurons of the cerebral cortex, hippocampus and subcortical regions. Cereb Cortex 2002;12(12):1298-311. doi: 10.1093/cercor/12.12.1298 [published Online First: 2002/11/13]

102. Amaral DG, Scharfman HE, Lavenex P. The dentate gyrus: fundamental neuroanatomical organization (dentate gyrus for dummies). Prog Brain Res 2007;163:3-22. doi: 10.1016/S0079-6123(07)63001-5 [published Online First: 2007/09/04]

103. Butterfield DA, Lauderback CM. Lipid peroxidation and protein oxidation in Alzheimer's disease brain: potential causes and consequences involving amyloid beta-peptide-associated free radical oxidative stress. Free Radic Biol Med 2002;32(11):1050-60. doi: 10.1016/s0891-5849(02)00794-3 [published Online First: 2002/05/29]

104. Perry G, Nunomura A, Hirai K, et al. Is oxidative damage the fundamental pathogenic mechanism of Alzheimer's and other neurodegenerative diseases? Free Radic Biol Med 2002;33(11):1475-9. doi: 10.1016/s08915849(02)01113-9 [published Online First: 2002/11/26]

105. Smith MA, Sayre LM, Monnier VM, et al. Oxidative posttranslational modifications in Alzheimer disease. A possible pathogenic role in the formation of senile plaques and neurofibrillary tangles. Mol Chem Neuropathol 1996;28(1-3):41-8. doi: 10.1007/BF02815203 [published Online First: 1996/05/01]

106. Sastry PS. Lipids of nervous tissue: composition and metabolism. Prog Lipid Res 1985;24(2):69-176. doi: 10.1016/0163-7827(85)90011-6 [published Online First: 1985/01/01]

107. Bazinet RP, Laye S. Polyunsaturated fatty acids and their metabolites in brain function and disease. Nat Rev Neurosci 2014;15(12):771-85. doi: 10.1038/nrn3820 [published Online First: 2014/11/13]

108. Bassett CN, Neely MD, Sidell KR, et al. Cerebrospinal fluid lipoproteins are more vulnerable to oxidation in Alzheimer's disease and are neurotoxic when oxidized ex vivo. Lipids 1999;34(12):1273-80. doi: 10.1007/s11745999-0478-1 [published Online First: 2000/02/01]

109. Pedersen WA, Chan SL, Mattson MP. A mechanism for the neuroprotective effect of apolipoprotein E: isoformspecific modification by the lipid peroxidation product 4-hydroxynonenal. J Neurochem 2000;74(4):1426-33. doi: 10.1046/j.1471-4159.2000.0741426.x [published Online First: 2000/03/29]

110. Butterfield DA, Mattson MP. Apolipoprotein E and oxidative stress in brain with relevance to Alzheimer's disease. Neurobiol Dis 2020;138:104795. doi: 10.1016/j.nbd.2020.104795 [published Online First: 2020/02/10]

111. Bassett CN, Swift LL, Montine KS, et al. Cerebrospinal fluid lipoprotein delivery to human neuronal cells is increased in Alzheimer's disease and is dependent on apoE monomer concentration. J Alzheimers Dis 2002;4(1):19-30. doi: 10.3233/jad-2002-4103 [published Online First: 2002/09/06]

112. D'Arcangelo G, Miao GG, Chen SC, et al. A protein related to extracellular matrix proteins deleted in the mouse mutant reeler. Nature 1995;374(6524):719-23. doi: 10.1038/374719a0 [published Online First: 1995/04/20]

113. Barr AM, MacLaurin SA, Semenova S, et al. Altered performance of reelin-receptor ApoER2 deficient mice on spatial tasks using the Barnes maze. Behav Neurosci 2007;121(5):1101-5. doi: 10.1037/0735-7044.121.5.1101 [published Online First: 2007/10/03]

114. Cuchillo-Ibanez I, Balmaceda V, Mata-Balaguer T, et al. Reelin in Alzheimer's Disease, Increased Levels but Impaired Signaling: When More is Less. J Alzheimers Dis 2016;52(2):403-16. doi: 10.3233/JAD-151193 [published Online First: 2016/04/01]

115. Chin J, Massaro CM, Palop JJ, et al. Reelin depletion in the entorhinal cortex of human amyloid precursor protein transgenic mice and humans with Alzheimer's disease. J Neurosci 2007;27(11):2727-33. doi: 10.1523/JNEUROSCI.3758-06.2007 [published Online First: 2007/03/16] 
medRxiv preprint doi: https://doi.org/10.1101/2021.07.05.21259649; this version posted July 6 , 2021. The copyright holder for this preprint

(which was not certified by peer review) is the author/funder, who has granted medRxiv a license to display the preprint in perpetuity. This article is a US Government work. It is not subject to copyright under 17 USC 105 and is also made available for use under a CCO license.

116. Herring A, Donath A, Steiner KM, et al. Reelin depletion is an early phenomenon of Alzheimer's pathology. J Alzheimers Dis 2012;30(4):963-79. doi: 10.3233/JAD-2012-112069 [published Online First: 2012/04/13]

117. Kubo K, Mikoshiba K, Nakajima K. Secreted Reelin molecules form homodimers. Neurosci Res 2002;43(4):381-8. doi: 10.1016/s0168-0102(02)00068-8 [published Online First: 2002/07/24]

118. Hattori M, Kohno T. Regulation of Reelin functions by specific proteolytic processing in the brain. J Biochem 2021 doi: 10.1093/jb/mvab015 [published Online First: 2021/02/11]

119. Urbanska M, Swiech L, Jaworski J. Developmental plasticity of the dendritic compartment: focus on the cytoskeleton. Adv Exp Med Biol 2012;970:265-84. doi: 10.1007/978-3-7091-0932-8_12 [published Online First: 2012/02/22]

120. Ben Zablah Y, Merovitch N, Jia Z. The Role of ADF/Cofilin in Synaptic Physiology and Alzheimer's Disease. Front Cell Dev Biol 2020;8:594998. doi: 10.3389/fcell.2020.594998 [published Online First: 2020/12/08]

121. Kang DE, Woo JA. Cofilin, a Master Node Regulating Cytoskeletal Pathogenesis in Alzheimer's Disease. J Alzheimers Dis 2019;72(s1):S131-S44. doi: 10.3233/JAD-190585 [published Online First: 2019/10/09]

122. Rush T, Martinez-Hernandez J, Dollmeyer M, et al. Synaptotoxicity in Alzheimer's Disease Involved a Dysregulation of Actin Cytoskeleton Dynamics through Cofilin 1 Phosphorylation. J Neurosci 2018;38(48):10349-61. doi: 10.1523/JNEUROSCI.1409-18.2018 [published Online First: 2018/10/21]

123. Bamburg JR, Bernstein BW. Actin dynamics and cofilin-actin rods in alzheimer disease. Cytoskeleton (Hoboken) 2016;73(9):477-97. doi: 10.1002/cm.21282 [published Online First: 2016/02/14]

124. Heredia L, Helguera P, de Olmos S, et al. Phosphorylation of actin-depolymerizing factor/cofilin by LIM-kinase mediates amyloid beta-induced degeneration: a potential mechanism of neuronal dystrophy in Alzheimer's disease. J Neurosci 2006;26(24):6533-42. doi: 10.1523/JNEUROSCI.5567-05.2006 [published Online First: 2006/06/16]

125. Rossi D, Gruart A, Contreras-Murillo G, et al. Reelin reverts biochemical, physiological and cognitive alterations in mouse models of Tauopathy. Prog Neurobiol 2020;186:101743. doi: 10.1016/j.pneurobio.2019.101743 [published Online First: 2019/12/25]

126. Scheff SW, Price DA, Schmitt FA, et al. Hippocampal synaptic loss in early Alzheimer's disease and mild cognitive impairment. Neurobiol Aging 2006;27(10):1372-84. doi: 10.1016/j.neurobiolaging.2005.09.012 [published Online First: 2005/11/18]

127. Ittner LM, Ke YD, Delerue F, et al. Dendritic function of tau mediates amyloid-beta toxicity in Alzheimer's disease mouse models. Cell 2010;142(3):387-97. doi: 10.1016/j.cell.2010.06.036 [published Online First: 2010/07/27]

128. Mondragon-Rodriguez S, Trillaud-Doppia E, Dudilot A, et al. Interaction of endogenous tau protein with synaptic proteins is regulated by N-methyl-D-aspartate receptor-dependent tau phosphorylation. J Biol Chem 2012;287(38):32040-53. doi: 10.1074/jbc.M112.401240 [published Online First: 2012/07/27]

129. Prikas $\mathrm{E}$, Ahel H, Stefanoska K, et al. Interaction between the guanylate kinase domain of PSD-95 and the prolinerich region and microtubule binding repeats 2 and 3 of tau. Biochem Cell Biol 2021 doi: 10.1139/bcb-2020-0604 [published Online First: 2021/04/02]

130. Tezuka T, Umemori H, Akiyama T, et al. PSD-95 promotes Fyn-mediated tyrosine phosphorylation of the N-methylD-aspartate receptor subunit NR2A. Proc Natl Acad Sci U S A 1999;96(2):435-40. doi: 10.1073/pnas.96.2.435 [published Online First: 1999/01/20]

131. Nakazawa T, Komai S, Tezuka T, et al. Characterization of Fyn-mediated tyrosine phosphorylation sites on GluR epsilon 2 (NR2B) subunit of the N-methyl-D-aspartate receptor. J Biol Chem 2001;276(1):693-9. doi: 10.1074/jbc.M008085200 [published Online First: 2000/10/12]

132. Ittner A, Chua SW, Bertz J, et al. Site-specific phosphorylation of tau inhibits amyloid-beta toxicity in Alzheimer's mice. Science 2016;354(6314):904-08. doi: 10.1126/science.aah6205 [published Online First: 2016/11/20]

133. Ittner A, Asih PR, Tan ARP, et al. Reduction of advanced tau-mediated memory deficits by the MAP kinase p38gamma. Acta Neuropathol 2020;140(3):279-94. doi: 10.1007/s00401-020-02191-1 [published Online First: 2020/07/30]

134. Arnaud L, Ballif BA, Forster E, et al. Fyn tyrosine kinase is a critical regulator of disabled-1 during brain development. Curr Biol 2003;13(1):9-17. doi: 10.1016/s0960-9822(02)01397-0 [published Online First: 2003/01/16]

135. Kuo G, Arnaud L, Kronstad-O'Brien P, et al. Absence of Fyn and Src causes a reeler-like phenotype. J Neurosci 2005;25(37):8578-86. doi: 10.1523/JNEUROSCI.1656-05.2005 [published Online First: 2005/09/16] 
medRxiv preprint doi: https://doi.org/10.1101/2021.07.05.21259649; this version posted July 6, 2021. The copyright holder for this preprint

(which was not certified by peer review) is the author/funder, who has granted medRxiv a license to display the preprint in perpetuity.

This article is a US Government work. It is not subject to copyright under 17 USC 105 and is also made available for use under a CCO license.

136. Snyder EM, Nong Y, Almeida CG, et al. Regulation of NMDA receptor trafficking by amyloid-beta. Nat Neurosci 2005;8(8):1051-8. doi: 10.1038/nn1503 [published Online First: 2005/07/19]

137. Cataldo AM, Peterhoff CM, Troncoso JC, et al. Endocytic pathway abnormalities precede amyloid beta deposition in sporadic Alzheimer's disease and Down syndrome: differential effects of APOE genotype and presenilin mutations. Am J Pathol 2000;157(1):277-86. doi: 10.1016/s0002-9440(10)64538-5 [published Online First: 2000/07/06]

138. Van Acker ZP, Bretou M, Annaert W. Endo-lysosomal dysregulations and late-onset Alzheimer's disease: impact of genetic risk factors. Mol Neurodegener 2019;14(1):20. doi: 10.1186/s13024-019-0323-7 [published Online First: 2019/06/05]

139. Chen Y, Durakoglugil MS, Xian X, et al. ApoE4 reduces glutamate receptor function and synaptic plasticity by selectively impairing ApoE receptor recycling. Proc Natl Acad Sci U S A 2010;107(26):12011-6. doi: 10.1073/pnas.0914984107 [published Online First: 2010/06/16]

140. Xian X, Pohlkamp T, Durakoglugil MS, et al. Reversal of ApoE4-induced recycling block as a novel prevention approach for Alzheimer's disease. Elife 2018;7 doi: 10.7554/eLife.40048 [published Online First: 2018/10/31]

141. He X, Cooley K, Chung CH, et al. Apolipoprotein receptor 2 and X11 alpha/beta mediate apolipoprotein E-induced endocytosis of amyloid-beta precursor protein and beta-secretase, leading to amyloid-beta production. $J$ Neurosci 2007;27(15):4052-60. doi: 10.1523/JNEUROSCI.3993-06.2007 [published Online First: 2007/04/13]

142. Hoe HS, Tran TS, Matsuoka Y, et al. DAB1 and Reelin effects on amyloid precursor protein and ApoE receptor 2 trafficking and processing. J Biol Chem 2006;281(46):35176-85. doi: 10.1074/jbc.M602162200 [published Online First: 2006/09/05]

143. Pramatarova A, Chen K, Howell BW. A genetic interaction between the APP and Dab1 genes influences brain development. Mol Cell Neurosci 2008;37(1):178-86. doi: 10.1016/j.mcn.2007.09.008 [published Online First: 2007/11/22]

144. Hoe HS, Minami SS, Makarova A, et al. Fyn modulation of Dab1 effects on amyloid precursor protein and ApoE receptor 2 processing. J Biol Chem 2008;283(10):6288-99. doi: 10.1074/jbc.M704140200 [published Online First: 2007/12/20]

145. Lane-Donovan C, Herz J. ApoE, ApoE Receptors, and the Synapse in Alzheimer's Disease. Trends Endocrinol Metab 2017;28(4):273-84. doi: 10.1016/j.tem.2016.12.001 [published Online First: 2017/01/07]

146. Jaeger S, Pietrzik CU. Functional role of lipoprotein receptors in Alzheimer's disease. Curr Alzheimer Res 2008;5(1):15-25. doi: 10.2174/156720508783884675 [published Online First: 2008/02/22]

147. Marzolo MP, Bu G. Lipoprotein receptors and cholesterol in APP trafficking and proteolytic processing, implications for Alzheimer's disease. Semin Cell Dev Biol 2009;20(2):191-200. doi: 10.1016/j.semcdb.2008.10.005 [published Online First: 2008/12/02]

148. Cam JA, Bu G. Modulation of beta-amyloid precursor protein trafficking and processing by the low density lipoprotein receptor family. Mol Neurodegener 2006;1:8. doi: 10.1186/1750-1326-1-8 [published Online First: 2006/08/26]

149. Ayodele T, Rogaeva E, Kurup JT, et al. Early-Onset Alzheimer's Disease: What Is Missing in Research? Curr Neurol Neurosci Rep 2021;21(2):4. doi: 10.1007/s11910-020-01090-y [published Online First: 2021/01/20]

150. Kontush A, Berndt C, Weber W, et al. Amyloid-beta is an antioxidant for lipoproteins in cerebrospinal fluid and plasma. Free Radic Biol Med 2001;30(1):119-28. doi: 10.1016/s0891-5849(00)00458-5 [published Online First: 2001/01/03]

151. Kontush A, Donarski N, Beisiegel U. Resistance of human cerebrospinal fluid to in vitro oxidation is directly related to its amyloid-beta content. Free Radic Res 2001;35(5):507-17. doi: 10.1080/10715760100301521 [published Online First: 2002/01/05]

152. Kontush A. Alzheimer's amyloid-beta as a preventive antioxidant for brain lipoproteins. Cell Mol Neurobiol 2001;21(4):299-315. doi: 10.1023/a:1012629603390 [published Online First: 2002/01/05]

153. Balakrishnan R, Parthasarathy R, Sulkowski E. Alzheimer's beta-amyloid peptide: affinity for metal chelates. J Pept Res 1998;51(2):91-5. doi: 10.1111/j.1399-3011.1998.tb00624.x [published Online First: 1998/03/27]

154. Mouton-Liger F, Paquet C, Dumurgier J, et al. Oxidative stress increases BACE1 protein levels through activation of the PKR-elF2alpha pathway. Biochim Biophys Acta 2012;1822(6):885-96. doi: 10.1016/j.bbadis.2012.01.009 [published Online First: 2012/02/07] 
medRxiv preprint doi: https://doi.org/10.1101/2021.07.05.21259649; this version posted July 6 , 2021. The copyright holder for this preprint

(which was not certified by peer review) is the author/funder, who has granted medRxiv a license to display the preprint in perpetuity. This article is a US Government work. It is not subject to copyright under 17 USC 105 and is also made available for use under a CCO license.

155. Devi L, Alldred MJ, Ginsberg SD, et al. Sex- and brain region-specific acceleration of beta-amyloidogenesis following behavioral stress in a mouse model of Alzheimer's disease. Mol Brain 2010;3:34. doi: 10.1186/1756-6606-3-34 [published Online First: 2010/11/10]

156. Wang Y, Li M, Tang J, et al. Glucocorticoids facilitate astrocytic amyloid-beta peptide deposition by increasing the expression of APP and BACE1 and decreasing the expression of amyloid-beta-degrading proteases. Endocrinology 2011;152(7):2704-15. doi: 10.1210/en.2011-0145 [published Online First: 2011/05/12]

157. Vassar R, Kovacs DM, Yan R, et al. The beta-secretase enzyme BACE in health and Alzheimer's disease: regulation, cell biology, function, and therapeutic potential. J Neurosci 2009;29(41):12787-94. doi: 10.1523/JNEUROSCI.3657-09.2009 [published Online First: 2009/10/16]

158. Tan JL, Li QX, Ciccotosto GD, et al. Mild oxidative stress induces redistribution of BACE1 in non-apoptotic conditions and promotes the amyloidogenic processing of Alzheimer's disease amyloid precursor protein. PLoS One 2013;8(4):e61246. doi: 10.1371/journal.pone.0061246 [published Online First: 2013/04/25]

159. Mermelstein DJ, McCammon JA, Walker RC. pH-dependent conformational dynamics of beta-secretase 1: A molecular dynamics study. J Mol Recognit 2019;32(3):e2765. doi: 10.1002/jmr.2765 [published Online First: 2018/09/29]

160. Small SA, Simoes-Spassov S, Mayeux R, et al. Endosomal Traffic Jams Represent a Pathogenic Hub and Therapeutic Target in Alzheimer's Disease. Trends Neurosci 2017;40(10):592-602. doi: 10.1016/j.tins.2017.08.003 [published Online First: 2017/10/01]

161. Schippling S, Kontush A, Arlt S, et al. Increased lipoprotein oxidation in Alzheimer's disease. Free Radic Biol Med 2000;28(3):351-60. doi: 10.1016/s0891-5849(99)00247-6 [published Online First: 2000/03/04]

162. Strittmatter WJ, Weisgraber KH, Huang DY, et al. Binding of human apolipoprotein E to synthetic amyloid beta peptide: isoform-specific effects and implications for late-onset Alzheimer disease. Proc Natl Acad Sci U S A 1993;90(17):8098-102. doi: 10.1073/pnas.90.17.8098 [published Online First: 1993/09/01]

163. Sperling R, Henley D, Aisen PS, et al. Findings of Efficacy, Safety, and Biomarker Outcomes of Atabecestat in Preclinical Alzheimer Disease: A Truncated Randomized Phase 2b/3 Clinical Trial. JAMA Neurol 2021;78(3):293301. doi: 10.1001/jamaneurol.2020.4857 [published Online First: 2021/01/20]

164. Doody RS, Raman R, Farlow M, et al. A phase 3 trial of semagacestat for treatment of Alzheimer's disease. N Engl J Med 2013;369(4):341-50. doi: 10.1056/NEJMoa1210951 [published Online First: 2013/07/26]

165. Timmers M, Streffer JR, Russu A, et al. Pharmacodynamics of atabecestat (JNJ-54861911), an oral BACE1 inhibitor in patients with early Alzheimer's disease: randomized, double-blind, placebo-controlled study. Alzheimers Res Ther 2018;10(1):85. doi: 10.1186/s13195-018-0415-6 [published Online First: 2018/08/24]

166. Ries M, Sastre M. Mechanisms of Abeta Clearance and Degradation by Glial Cells. Front Aging Neurosci 2016;8:160. doi: 10.3389/fnagi.2016.00160 [published Online First: 2016/07/28]

167. Gomez-Arboledas A, Davila JC, Sanchez-Mejias E, et al. Phagocytic clearance of presynaptic dystrophies by reactive astrocytes in Alzheimer's disease. Glia 2018;66(3):637-53. doi: 10.1002/glia.23270 [published Online First: 2017/11/28]

168. Manelli AM, Stine WB, Van Eldik LJ, et al. ApoE and Abeta1-42 interactions: effects of isoform and conformation on structure and function. J Mol Neurosci 2004;23(3):235-46. doi: 10.1385/JMN:23:3:235 [published Online First: 2004/06/08]

169. Carter DB. The interaction of amyloid-beta with ApoE. Subcell Biochem 2005;38:255-72. doi: 10.1007/0-387-232265_13 [published Online First: 2005/02/16]

170. Plascencia-Villa G, Perry G. Status and future directions of clinical trials in Alzheimer's disease. Int Rev Neurobiol 2020;154:3-50. doi: 10.1016/bs.irn.2020.03.022 [published Online First: 2020/08/03]

171. Pikkarainen $M$, Martikainen $P$, Alafuzoff I. The effect of prolonged fixation time on immunohistochemical staining of common neurodegenerative disease markers. J Neuropathol Exp Neurol 2010;69(1):40-52. doi: 10.1097/NEN.0b013e3181c6c13d [published Online First: 2009/12/17]

172. Gundisch S, Grundner-Culemann K, Wolff C, et al. Delayed times to tissue fixation result in unpredictable global phosphoproteome changes. J Proteome Res 2013;12(10):4424-34. doi: 10.1021/pr400451z [published Online First: 2013/08/30]

173. Birdsill AC, Walker DG, Lue L, et al. Postmortem interval effect on RNA and gene expression in human brain tissue. Cell Tissue Bank 2011;12(4):311-8. doi: 10.1007/s10561-010-9210-8 [published Online First: 2010/08/13] 
medRxiv preprint doi: https://doi.org/10.1101/2021.07.05.21259649; this version posted July 6, 2021. The copyright holder for this preprint (which was not certified by peer review) is the author/funder, who has granted medRxiv a license to display the preprint in perpetuity. This article is a US Government work. It is not subject to copyright under 17 USC 105 and is also made available for use under a CCO license.

174. Walker DG, Whetzel AM, Serrano G, et al. Characterization of RNA isolated from eighteen different human tissues: results from a rapid human autopsy program. Cell Tissue Bank 2016;17(3):361-75. doi: 10.1007/s10561-0169555-8 [published Online First: 2016/04/17]

175. Nichols JB, Malek-Ahmadi M, Tariot PN, et al. Vascular Lesions, APOE epsilon4, and Tau Pathology in Alzheimer Disease. J Neuropathol Exp Neurol 2021;80(3):240-46. doi: 10.1093/jnen/nlaa160 [published Online First: 2021/02/23]

176. Maric D, Jahanipour J, Li XR, et al. Whole-brain tissue mapping toolkit using large-scale highly multiplexed immunofluorescence imaging and deep neural networks. Nat Commun 2021;12(1):1550. doi: 10.1038/s41467021-21735-x [published Online First: 2021/03/12]

177. Amunts K, Kedo O, Kindler M, et al. Cytoarchitectonic mapping of the human amygdala, hippocampal region and entorhinal cortex: intersubject variability and probability maps. Anat Embryol (Berl) 2005;210(5-6):343-52. doi: 10.1007/s00429-005-0025-5 [published Online First: 2005/10/07] 